\title{
The Lindenbaum algebra of the theory of the class of all finite models
}

\author{
Steffen Lempp* \\ Mikhail Peretyat'kin ${ }^{\dagger}$ Reed Solomon ${ }^{\ddagger}$
}

\begin{abstract}
In this paper, we investigate the Lindenbaum algebra $\mathcal{L}\left(T_{\text {fin }}\right)$ of the theory $T_{f i n}=\operatorname{Th}\left(M_{f i n}\right)$ of the class $M_{f i n}$ of all finite models of a finite rich signature. We prove that this algebra is an atomic Boolean algebra while its Gödel numeration $\gamma$ is a $\Pi_{1}^{0}$-numeration. Moreover, the quotient algebra $\left(\mathcal{L}\left(T_{\text {fin }}\right) / \mathcal{F}, \gamma / \mathcal{F}\right)$ modulo the Fréchet ideal $\mathcal{F}$ is a $\Sigma_{2}^{0}$-algebra, which is universal over the class of all $\Sigma_{2}^{0}$ Boolean algebras. These conditions characterize uniquely the algebra $\mathcal{L}\left(T_{\text {fin }}\right)$; moreover, these conditions characterize up to recursive isomorphism the numerated Boolean quotient algebra $\left(\mathcal{L}\left(T_{\text {fin }}\right) / \mathcal{F}, \gamma / \mathcal{F}\right)$.

These results extend the work of Trakhtenbrot [17] and Vaught [18] on the first order theory of the class of all finite models of a finite rich signature.
\end{abstract}

We consider theories in first-order predicate logic with equality and use general concepts of model theory, computability theory, Boolean algebras and constructive models found in Chang and Keisler [1], Soare [16], Goncharov and Ershov [4] and Goncharov [3].

In the description of a signature, the superscripts specify the arities of the appropriate symbols. A finite signature is called rich if it contains either an $n$-ary predicate or function symbol for $n>1$, or two unary function symbols. $S L(\sigma)$ denotes the set of all sentences of signature $\sigma$. The Lindenbaum algebra of the sentences of a theory $T$ is denoted by $\mathcal{L}(T)$ (in fact, it is a Boolean algebra). $M_{\text {fin }}(\sigma)$ denotes the class of all finite models of signature $\sigma$. By Card $X$, we denote the number of elements of a finite set $X$.

*Department of Mathematics, University of Wisconsin, Madison, WI 53706-1388, USA. This author was partially supported by NSF grant DMS-9732526.

$\dagger$ Kazakh Academy of Sciences, Institute of Mathematics, Alma-Ata 480100, Kazakhstan.

$\ddagger$ Department of Mathematics, University of Wisconsin, Madison, WI 53706-1388, USA. This author was partially supported by NSF grant DMS-0071586. 
If $\Phi$ is a sentence and $\sigma$ is the signature consisting of the symbols which occur in $\Phi$, then $[\Phi]$ denotes the theory of signature $\sigma$ generated by $\Phi$ as an axiom, $\operatorname{Mod}(\Phi)$ denotes the class of all models $\mathfrak{M}$ of signature $\sigma$ such that $\mathfrak{M} \models \Phi$, while $\operatorname{ModFin}(\Phi)=\operatorname{Mod}(\Phi) \cap \mathrm{M}_{\text {fin }}(\sigma)$. We often write $\mathcal{L}(\Phi)$ instead of $\mathcal{L}([\Phi])$, and $\mathcal{L}(M)$ instead of $\mathcal{L}(\operatorname{Th}(M))$ when $M$ is a class of models.

If $\Phi$ is a formula and $\alpha \in\{0,1\}$, then $\Phi^{\alpha}$ denotes $\Phi$ for $\alpha=1$ and $\urcorner \Phi$ for $\alpha=0$. If $b$ is an element of a Boolean algebra and $\alpha \in\{0,1\}$, then $b^{\alpha}$ denotes $b$ for $\alpha=1$ and $-b$ for $\alpha=0$.

The following technical facts hold:

Lemma 0.1. Let $\mathcal{B}$ be a Boolean algebra. Then

(a) for any $a, b \in \mathcal{B}$, we have $a=b$ if and only if $a^{\alpha} \cap b^{\beta}=0$ for all pairs $(\alpha, \beta) \in\{(1,0),(0,1)\}$.

(b) for any $a, b, c \in \mathcal{B}$, we have $a \cap b=c$ if and only if $a^{\alpha} \cap b^{\beta} \cap c^{\gamma}=0$ for all triples $(\alpha, \beta, \gamma) \in\{(1,1,0),(0,0,1),(0,1,1),(1,0,1)\}$.

(c) for any $a, b \in \mathcal{B}$, we have $-a=b$ if and only if $a^{\alpha} \cap b^{\beta}=0$ for all pairs $(\alpha, \beta) \in\{(0,0),(1,1)\}$.

Proof. By direct calculation.

A numeration or numbering of a finite or countable Boolean algebra $\mathcal{B}$ is a surjective mapping $\nu: \mathbb{N} \stackrel{\text { onto }}{\longrightarrow}|\mathcal{B}|$. An isomorphism $\lambda: \mathcal{B}_{1} \rightarrow \mathcal{B}_{2}$ is called a constructive isomorphism or a recursive isomorphism between numerated Boolean algebras $\left(\mathcal{B}_{1}, \nu_{1}\right)$ and $\left(\mathcal{B}_{2}, \nu_{2}\right)$ if there are recursive functions $g_{1}(x)$ and $g_{2}(x)$ such that $\lambda\left(\nu_{1}(x)\right)=\nu_{2}\left(g_{1}(x)\right)$ and $\nu_{2}(x)=\lambda\left(\nu_{1}\left(g_{2}(x)\right)\right)$ for all $x \in \mathbb{N}$, i.e., the following diagram is commutative:

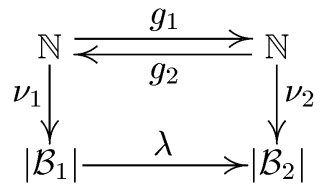

We write $\left(\mathcal{B}_{1}, \nu_{1}\right) \cong\left(\mathcal{B}_{2}, \nu_{2}\right)$ if these numerated Boolean algebras are isomorphic.

A numerated Boolean algebra $(\mathcal{B}, \nu)$ is called a $\Sigma_{n}^{0}$ algebra if all Boolean operations in $\mathcal{B}$ are representable by general recursive functions on numbers, while the equality relation is $\Sigma_{n}^{0}$ with respect to the numeration $\nu$. Similar notions apply to the hierarchies $\Pi_{n}^{0}$ and $\Delta_{n}^{0}$.

\section{The main results}

In this paper, we consider the class $M_{f i n}=M_{f i n}(\sigma)$ of all finite models of a fixed finite rich signature $\sigma$. We study the theory $T_{f i n}=\operatorname{Th}\left(M_{f i n}\right)$ of this class of models. The main result is a characterization of the Lindenbaum algebra 
$\left(\mathcal{L}\left(T_{\text {fin }}\right), \gamma\right)$, viewed as a numerated algebra together with its Gödel numeration $\gamma$. Note that a Gödel numeration always exists for the set $S L(\sigma)$ and therefore for the Lindenbaum algebra $\mathcal{L}\left(T_{\text {fin }}\right)$. For this reason, Gödel numerations are always assumed even when not mentioned explicitly.

The characterization of the Lindenbaum algebra is given by the following list of theorems.

Theorem 1.1. The Lindenbaum algebra $\left(\mathcal{L}\left(T_{\text {fin }}\right), \gamma\right)$ is a $\Pi_{1}^{0}$-algebra (i.e., it is a negatively numerated Boolean algebra).

Proof. For sentences $\Phi, \Psi \in S L(\sigma)$, the formula $\Phi \leftrightarrow \Psi$ is provable in $T_{\text {fin }}$ if and only if it is true in all finite models. This gives the $\Pi_{1}^{0}$ form for equality in the Boolean algebra $\mathcal{L}\left(T_{\text {fin }}\right)$ under the numeration $\gamma$.

Theorem 1.2. There exists a numeration $\nu^{*}$ such that $\left(\mathcal{L}\left(T_{\text {fin }}\right), \nu^{*}\right)$ is a constructive Boolean algebra.

Proof. By Odintsov and Selivanov [9], if a Boolean algebra has a negative numeration, i.e., $\Pi_{1}^{0}$-numeration, then it has a constructive one. Theorem 1.2 directly follows from this fact.

Theorem 1.3. The algebra $\mathcal{L}\left(T_{\text {fin }}\right)$ is a countable atomic Boolean algebra.

Proof. If $\Phi \in S L(\sigma)$ and $[\Phi]_{T_{\text {fin }}} \neq 0$, then $\Phi$ has a finite model $\mathfrak{M}$. Consider a sentence $\Psi$ of the form $(\exists \bar{x})[\mathcal{A D}(\bar{x}) \&(\forall y)(y \in \bar{x})]$ where $\mathcal{A D}(\bar{x})$ describes the complete atomic diagram of the model $\mathfrak{M}$. In the Boolean algebra $\mathcal{L}\left(T_{\text {fin }}\right)$, we have that $[\Psi]_{T_{f i n}} \leqslant[\Phi]_{T_{f i n}}$ and $[\Psi]_{T_{f i n}}$ is an atom.

Let $\mathcal{F}$ denote the Fréchet ideal of the algebra $\mathcal{L}\left(T_{\text {fin }}\right)$, i.e., the ideal generated by its atoms. Throughout this paper, $\mathcal{F}$ will be used to denote the Fréchet ideal. For a numerated Boolean algebra $(\mathcal{B}, \nu)$, the numerated quotient algebra $(\mathcal{B} / \mathcal{F}(\mathcal{B}), \nu / \mathcal{F}(\mathcal{B}))$ modulo $\mathcal{F}(\mathcal{B})$ is defined as follows: $\mathcal{B} / \mathcal{F}(\mathcal{B})$ is the ordinary quotient algebra, and $\nu / \mathcal{F}(\mathcal{B})$ is a numeration of this quotient algebra generated by the numbering $\nu$. ideal.

The following properties hold for the quotient algebra modulo the Fréchet

Theorem 1.4. The algebra $\left(\mathcal{L}\left(T_{\text {fin }}\right) / \mathcal{F}, \gamma / \mathcal{F}\right)$ is a $\Sigma_{2}^{0}$ Boolean algebra.

Proof. One can easily see that all Boolean operations are represented by recursive functions on numbers. Furthermore, we have for the equality relation that

$$
\begin{aligned}
& \gamma / \mathcal{F}(m)=\gamma / \mathcal{F}(n) \Leftrightarrow \\
& (\exists k)[\gamma(m) \leftrightarrow \gamma(n) \text { is true in all finite models of size }>k],
\end{aligned}
$$

which yields a quantifier prefix of the form $\exists \forall$.

The main theorem of this article is given by the following statement.

Theorem 1.5. [Main Theorem] Let $(\mathcal{B}, \nu)$ be an arbitrary $\Sigma_{2}^{0}$ Boolean al- 
gebra. Then there exists a formula $\Omega$ of a the finite rich signature $\sigma_{0}$ such that there is a recursive isomorphism of the form

$$
(\mathcal{B}, \nu) \cong(\mathcal{L}(\operatorname{ModFin}(\Omega)) / \mathcal{F}, \gamma / \mathcal{F}) .
$$

Thus, any $\Sigma_{2}^{0}$ Boolean algebra can be realized as the quotient algebra of the Lindenbaum algebra (modulo the Fréchet ideal) of the theory of the class of all finite models of a sentence $\Omega$ of a finite rich signature $\sigma_{0}$. We prove this statement in the remainder of this paper, starting in Section 2.

In [13], a signature reduction procedure Reduct is described which satisfies the properties listed below. Let $\Theta^{\prime}$ be a sentence of a signature $\sigma^{\prime}$, and $\sigma^{\prime \prime}$ be any finite rich signature. Given $\Theta^{\prime}$ and $\sigma^{\prime \prime}$, the procedure Reduct yields a sentence $\Theta^{\prime \prime}=\operatorname{Reduct}\left(\Theta^{\prime}, \sigma^{\prime \prime}\right)$ of the signature $\sigma^{\prime \prime}$ and a recursive isomorphism $\mu: \mathcal{L}\left(\Theta^{\prime}\right) \rightarrow \mathcal{L}\left(\Theta^{\prime \prime}\right)$ between Lindenbaum algebras of theories generated by these sentences such that $\mu$ preserves the model-theoretic property

$$
p=\text { "there exists a finite model," }
$$

as well as a lot of other properties that are not of interest in this paper. The preservation of property $p$ means that for any complete extension $T^{\prime}$ of theory $\left[\Theta^{\prime}\right]$, and corresponding complete extension $T^{\prime \prime}$ of theory $\left[\Theta^{\prime \prime}\right]$, if $T^{\prime \prime}=\mu\left(T^{\prime}\right)$, then the theory $T^{\prime \prime}$ has a finite model if and only if the theory $T^{\prime}$ has a finite model.

The signature reduction procedure Reduct makes the statement of Theorem 1.5 valid for any finite rich signature, in particular for the signature $\sigma$ which we are considering:

Theorem 1.6. Let $(\mathcal{B}, \nu)$ be an arbitrary $\Sigma_{2}^{0}$ Boolean algebra. Then there exists a formula $\Theta$ of a given finite rich signature $\sigma$ such that there is a recursive isomorphism of the form $(\mathcal{B}, \nu) \cong(\mathcal{L}(\operatorname{ModFin}(\Theta)) / \mathcal{F}, \gamma / \mathcal{F})$.

Proof. Fix a numerated $\Sigma_{2}^{0}$ Boolean algebra $(\mathcal{B}, \nu)$. By Theorem 1.5, there is a sentence $\Omega$ of a finite rich signature $\sigma_{0}$ satisfying

$$
(\mathcal{B}, \nu) \cong(\mathcal{L}(\operatorname{ModFin}(\Omega)) / \mathcal{F}, \gamma / \mathcal{F}) .
$$

Consider the sentence $\Theta=\operatorname{Reduct}(\Omega, \sigma)$ of the signature $\sigma$. The procedure Reduct provides a recursive isomorphism between the Lindenbaum algebras of the theories generated by these sentences, $\mu: \mathcal{L}(\Omega) \rightarrow \mathcal{L}(\Theta)$, which preserves the property $p$ (the existence of a finite model). From this, one can show that $\mu$ is a recursive isomorphism between Lindenbaum algebras $\mathcal{L}(\operatorname{ModFin}(\Omega))$ and $\mathcal{L}(\operatorname{ModFin}(\Theta))$. Furthermore, $\mu$ is a recursive isomorphism between their quotients modulo the Fréchet ideals. Thus, we have

$$
(\mathcal{B}, \nu) \cong(\mathcal{L}(\operatorname{ModFin}(\Theta)) / \mathcal{F}, \gamma / \mathcal{F})
$$


for a sentence $\Theta$ of signature $\sigma$, so we obtain exactly the property we need.

Let $\mathcal{B}$ be a Boolean algebra and $a$ an element of $\mathcal{B} . \quad \mathcal{B}[a]$ will denote the Boolean algebra of elements below $a$. Formally, $\mathcal{B}[a]$ is defined on the set $[0, a]=$ $\{x \mid 0 \subseteq x \subseteq a\}$, inherits $\cup, \cap$ and 0 from $\mathcal{B}$, uses $a$ as the unit element, and takes the relative complement in the interval $[0, a]$ of $\mathcal{B}$ as the complement operation (i.e., the complement in $\mathcal{B}[a]$ is defined by the rule $-x=a \backslash x$ ). One can easily check that this structure is a Boolean algebra. It is called the restriction of $\mathcal{B}$ to the element $a$.

Now let $(\mathcal{B}, \nu)$ be a numerated Boolean algebra and $a=\nu\left(n_{0}\right)$ be an element of $\mathcal{B}$. We let $(\mathcal{B}, \nu)[a]$ denote the numerated Boolean algebra $\left(\mathcal{B}^{\prime}, \nu^{\prime}\right)$ such that $\mathcal{B}^{\prime}=\mathcal{B}[a]$ and $\nu^{\prime}$ is a numeration of the Boolean algebra $\mathcal{B}[a]$ which is induced from the numeration $\nu$ by the rule $\nu^{\prime}(n)=\nu(n) \wedge \nu\left(n_{0}\right)$. We denote this numeration $\nu^{\prime}$ by $\nu[a]$. So the introduced operation has the form $(\mathcal{B}, \nu)[a]=$ $(\mathcal{B}[a], \nu[a])$.

Theorem 1.7. For any $\Sigma_{2}^{0}$ Boolean algebra $(\mathcal{B}, \nu)$ there is an element $a \in$ $\mathcal{L}\left(T_{\text {fin }}\right) / \mathcal{F}$ and a recursive isomorphism $(\mathcal{B}, \nu) \cong\left(\mathcal{L}\left(T_{\text {fin }}\right) / \mathcal{F}, \gamma / \mathcal{F}\right)[a]$.

Proof. Immediate by Theorem 1.6 since

$$
\left(\mathcal{L}\left(T_{\text {fin }}\right) / \mathcal{F}, \gamma / \mathcal{F}\right)\left[[\Phi]_{\mathcal{F}}\right] \cong(\mathcal{L}(\operatorname{ModFin}(\Phi)) / \mathcal{F}, \gamma / \mathcal{F})
$$

Selivanov has proved in [14] and [15] that $\Sigma_{n}^{0}$-universal Boolean algebras exist for all classes $\Sigma_{n}^{0}$ of the arithmetical hierarchy; moreover, they are defined uniquely up to constructive isomorphism. By Selivanov's method, we prove below that the $\Sigma_{2}^{0}$-universal Boolean algebra exists and is defined uniquely up to constructive isomorphism.

Let $(\mathfrak{B}, \mu)$ be a countable constructive atomless Boolean algebra and let $W_{n}^{\prime}, n \in \mathbb{N}$, be a Post enumeration of all sets of integers which are recursively enumerable in the oracle $\varnothing^{\prime}$. Denote by $\mathcal{F}_{m}^{\prime}$ the ideal of $\mathfrak{B}$ generated by the set $\left\{\mu(k) \mid k \in W_{m}^{\prime}\right\}$.

Theorem 1.8. The following assertions hold:

(a) For any $m \in \mathbb{N}, \mathcal{F}_{m}^{\prime}$ is a $\Sigma_{2}^{0}$-ideal and $\left(\mathfrak{B} / \mathcal{F}_{m}^{\prime}, \mu / \mathcal{F}_{m}^{\prime}\right)$ is a $\Sigma_{2}^{0}$ Boolean algebra.

(b) For any $\Sigma_{2}^{0}$ Boolean algebra $(\mathcal{B}, \nu)$ there is $m \in \mathbb{N}$ such that the following constructive isomorphism holds: $(\mathcal{B}, \nu) \cong\left(\mathfrak{B} / \mathcal{F}_{m}^{\prime}, \mu / \mathcal{F}_{m}^{\prime}\right)$.

(c) The direct product of the numerated Boolean algebras

$$
\left(\mathfrak{B}^{*}, \mu^{*}\right)=\bigotimes_{m \in \mathbb{N}}\left(\mathfrak{B} / \mathcal{F}_{m}^{\prime}, \mu / \mathcal{F}_{m}^{\prime}\right)
$$

is a $\Sigma_{2}^{0}$ Boolean algebra. 
Proof. By inspection of the standard definitions.

Theorem 1.9. The following representation of the Lindenbaum algebra of the theory $T_{\text {fin }}$ of the class of all finite models modulo the Fréchet ideal holds:

$$
\left(\mathcal{L}\left(T_{\text {fin }}\right) / \mathcal{F}, \gamma / \mathcal{F}\right) \cong\left(\mathfrak{B}^{*}, \mu^{*}\right) \cong \bigotimes_{m \in \mathbb{N}}\left(\mathfrak{B} / \mathcal{F}_{m}^{\prime}, \mu / \mathcal{F}_{m}^{\prime}\right)
$$

Proof. (due to V.L. Selivanov) By Theorem $1.8,\left(\mathfrak{B}^{*}, \mu^{*}\right)$ is a $\Sigma_{2}^{0}$ Boolean algebra, and therefore, by Theorem 1.7, there exists an element $a \in \mathcal{L}\left(T_{\text {fin }}\right) / \mathcal{F}$ such that $\left(\mathcal{L}\left(T_{\text {fin }}\right) / \mathcal{F}, \gamma / \mathcal{F}\right)[a] \cong\left(\mathfrak{B}^{*}, \mu^{*}\right)$. On the other hand, we have the following natural decomposition of the Boolean algebra by an element and its complement:

$$
\left(\mathcal{L}\left(T_{\text {fin }}\right) / \mathcal{F}, \gamma / \mathcal{F}\right) \cong\left(\mathcal{L}\left(T_{\text {fin }}\right) / \mathcal{F}, \gamma / \mathcal{F}\right)[-a] \otimes\left(\mathcal{L}\left(T_{\text {fin }}\right) / \mathcal{F}, \gamma / \mathcal{F}\right)[a] .
$$

In this expression, $\left(\mathcal{L}\left(T_{\text {fin }}\right) / \mathcal{F}, \gamma / \mathcal{F}\right)[-a]$ is a $\Sigma_{2}^{0}$ Boolean algebra; therefore, there exists $k \in \mathbb{N}$ such that this algebra is isomorphic to $\left(\mathfrak{B} / \mathcal{F}_{k}^{\prime}, \mu / \mathcal{F}_{k}^{\prime}\right)$. From this, we obtain the following decomposition:

$$
\left(\mathcal{L}\left(T_{\text {fin }}\right) / \mathcal{F}, \gamma / \mathcal{F}\right) \cong\left(\mathfrak{B} / \mathcal{F}_{k}^{\prime}, \mu / \mathcal{F}_{k}^{\prime}\right) \otimes \bigotimes_{m \in \mathbb{N}}\left(\mathfrak{B} / \mathcal{F}_{m}^{\prime}, \mu / \mathcal{F}_{m}^{\prime}\right)
$$

One can see that by the Padding Lemma [16], the numeration $W_{n}^{\prime}, n \in \mathbb{N}$, is an effective cylinder. Therefore, we can construct a general recursive function $p: \mathbb{N} \rightarrow \mathbb{N}$ such that $p$ is a permutation of $\mathbb{N}, p(0)=k$, and $W_{p(n+1)}^{\prime}=W_{n}^{\prime}$ for all $n \in \mathbb{N}$. We can now infer

$$
\begin{aligned}
& \left(\mathfrak{B}^{*}, \mu^{*}\right) \cong \bigotimes_{m \in \mathbb{N}}\left(\mathfrak{B} / \mathcal{F}_{m}^{\prime}, \mu / \mathcal{F}_{m}^{\prime}\right) \cong \bigotimes_{m \in \mathbb{N}}\left(\mathfrak{B} / \mathcal{F}_{p(m)}^{\prime}, \mu / \mathcal{F}_{p(m)}^{\prime}\right) \cong \\
& \left(\mathfrak{B} / \mathcal{F}_{p(0)}^{\prime}, \mu / \mathcal{F}_{p(0)}^{\prime}\right) \otimes \bigotimes_{m \in \mathbb{N}}\left(\mathfrak{B} / \mathcal{F}_{p(m+1)}^{\prime}, \mu / \mathcal{F}_{p(m+1)}^{\prime}\right) \cong \\
& \left(\mathfrak{B} / \mathcal{F}_{k}^{\prime}, \mu / \mathcal{F}_{k}^{\prime}\right) \otimes \bigotimes_{m \in \mathbb{N}}\left(\mathfrak{B} / \mathcal{F}_{m}^{\prime}, \mu / \mathcal{F}_{m}^{\prime}\right) \cong \\
& \left(\mathcal{L}\left(T_{\text {fin }}\right) / \mathcal{F}, \gamma / \mathcal{F}\right)
\end{aligned}
$$

as desired.

In [10] it was proved that for any two countable atomic Boolean algebras $\mathcal{B}_{1}$ and $\mathcal{B}_{2}$, if their quotient algebras modulo the Fréchet ideal, $\mathcal{B}_{1} / \mathcal{F}\left(\mathcal{B}_{1}\right)$ and $\mathcal{B}_{2} / \mathcal{F}\left(\mathcal{B}_{2}\right)$, are isomorphic, then the original algebras are isomorphic: $\mathcal{B}_{1} \cong \mathcal{B}_{2}$. Thus, Theorems 1.3 and 1.9 characterize the abstract isomorphism type of the algebra $\mathcal{L}\left(T_{\text {fin }}\right)$. As for the characterization of the isomorphism type of $\left(\mathcal{L}\left(T_{\text {fin }}\right), \gamma\right)$ considered as a numerated algebra, this question remains open.

Now we start the proof of Theorem 1.5, to which the remainder of this paper is devoted. 


\section{Two numerations of tuples}

The canonical (Gödel) index of the finite tuple $\varepsilon=\left\langle\varepsilon_{0}, \varepsilon_{1}, \ldots, \varepsilon_{m-1}\right\rangle$, with $\varepsilon_{i} \in\{0,1\}$, is the number

$$
\begin{aligned}
\operatorname{Nom}(\varepsilon) & =2^{m}+\varepsilon_{0} 2^{m-1}+\varepsilon_{1} 2^{m-2}+\ldots+\varepsilon_{m-1}-1 \\
& =\left(1 \varepsilon_{0} \varepsilon_{1} \ldots \varepsilon_{m-1}\right)_{2}-1
\end{aligned}
$$

where the subindex 2 means that $1 \varepsilon_{0} \varepsilon_{1} \ldots \varepsilon_{m-1}$ is a number defined in the binary representation. It is easy to see that the function Nom thus defined represents a one-to-one correspondence between the set $2^{<\omega}$ of all finite tuples of zeros and ones and the set $\mathbb{N}$ of all natural numbers. We will use the notation \langle\rangle for the empty string.

The following recursive relation for the function Nom

$$
\begin{aligned}
& \operatorname{Nom}\langle\rangle=0, \\
& \operatorname{Nom}\left\langle\varepsilon_{0}, \varepsilon_{1}, \ldots, \varepsilon_{m}\right\rangle=2 \cdot \operatorname{Nom}\left\langle\varepsilon_{0}, \varepsilon_{1}, \ldots, \varepsilon_{m-1}\right\rangle+\varepsilon_{m}+1, m \geqslant 0,
\end{aligned}
$$

follows directly from equation (2.1). In fact, the recursive relation (2.2) could be used as the basic definition, with the relation (2.1) then being its corollary.

The following properties of the numeration function Nom

$$
\begin{aligned}
& m \leqslant 2^{m}-1 \leqslant \operatorname{Nom}\left\langle\varepsilon_{0}, \varepsilon_{1}, \ldots, \varepsilon_{m-1}\right\rangle \leqslant 2^{m+1}-2, \text { for all } m \in \mathbb{N}, \varepsilon_{i} \in\{0,1\}, \\
& \operatorname{Nom}(\varepsilon)<\operatorname{Nom}\left(\varepsilon^{\prime}\right), \text { for all } \varepsilon, \varepsilon^{\prime} \in 2^{<\omega} \text { such that }|\varepsilon|<\left|\varepsilon^{\prime}\right|,
\end{aligned}
$$

can be deduced from Definition (2.1). As for (2.3), we have for the sequences of length $m$ with all zeros or ones

$$
\operatorname{Nom}\langle 0,0, \ldots, 0\rangle=2^{m}-1, \quad \operatorname{Nom}\langle 1,1, \ldots, 1\rangle=2^{m+1}-2,
$$

while numbers of other sequences of length $m$ must be between these values.

The particular function Nom introduced above is used in the construction of finitely axiomatizable theories as a numeration function for the set of all tuples of zeros and ones of finite length.

We use the standard pairing function defined by

$$
c(x, y)=\frac{x^{2}+2 x y+y^{2}+3 x+y}{2} .
$$

One can check that the function $c(x, y)$ satisfies the following properties

$$
\begin{aligned}
& (x, y)=(u, v) \Leftrightarrow c(x, y)=c(u, v) \Leftrightarrow c^{2}(x, y)=c^{2}(u, v), \\
& \text { for all } x, y, u, v \geqslant 0, \\
& c(x, y) \geqslant|x|+|y|, \text { for all } x, y \geqslant 0 .
\end{aligned}
$$




\section{Computations unfolded in time on a Turing machine}

In this section, we describe the type of Turing machines used in the construction of a finitely axiomatizable theory.

A machine $\mathcal{M}$ has a finite set $\left\{a_{i} \mid i<d\right\}$ of tape symbols and a finite set $\left\{q_{j} \mid j<e\right\}$ of state symbols. The program of the machine consists of a finite set of commands of the form

$$
\begin{aligned}
& a_{i} q_{j} \rightarrow a_{m} q_{t} L, \\
& a_{i} q_{j} \rightarrow a_{m} q_{t} R .
\end{aligned}
$$

The tape of the machine is two-way infinite and is divided into cells. At each stage, the head of the machine scans one cell of the tape. Each cell contains exactly one symbol from $\left\{a_{i} \mid i<d\right\}$ (Fig. 3.1). At each stage the machine is in one of the states from $\left\{q_{j} \mid j<e\right\}$. The machine starts in state $q_{0}$. The command (3.1) means that if at some stage the machine is in state $q_{j}$ and reads symbol $a_{i}$ in the current cell, then the machine writes $a_{m}$ in the current cell, goes into state $q_{t}$ and moves its head to the adjacent cell on the left. The command (3.2) is similar except that the machine moves its head to the right. We say that this command applies to the situation $a_{i} q_{j}$. We require that in any situation no more than one command applies. The machine stops when no command applies. Thus, the operation of a machine is uniquely determined by its program and the information on the tape at the initial stage.

$$
\begin{array}{l|l|l|l|l|l|l|l|l|l|l}
\cline { 3 - 6 } & \multicolumn{10}{c|}{q_{j}} \\
\hline \cdots & a_{h} & a_{k} & a_{l} & a_{i} & a_{n} & a_{p} & a_{q} & a_{r} & a_{s} & \cdots \\
\hline
\end{array}
$$

Fig. 3.1. Working process of a Turing machine

We have not specified the output of the Turing machine because for our purposes, only the process of the operation of the Turing machine will be relevant, and not the output. In some cases, the program of some other Turing machines is used as a subprogram. In such cases, the form of the input and the output of the subprogram is to be defined by additional specifications.

Let $S T_{d}$ denote the set of all possible initial configurations of a machine with $d$ tape symbols. Since the tape cells have the same order type as the integers, since each cell contains one of the symbols $a_{0}, a_{1}, \ldots, a_{d-1}$, and since the machine always starts in state $q_{0}$, if we associate the starting position of the head with the position $0 \in \mathbb{Z}$, we obtain that each configuration $\mathcal{S} \in S T_{d}$ can be represented by a map $\mathcal{S}: \mathbb{Z} \rightarrow\{0,1, \ldots, d-1\}$. If $\mathcal{S}$ has this form, then $\mathcal{S}(k)$, $k \in \mathbb{Z}$, denotes the index of the symbol on the tape in the $k$-th cell, counting from the initial position of the head. If $\mathcal{S} \in S T_{d}$ then an $\mathcal{S}$-computation on the machine $\mathcal{M}$ will be the operation of $\mathcal{M}$ starting from $\mathcal{S}$. 
We call the set of all Turing machines described above with a fixed parameter $d$ and arbitrary $e$ the class of Turing machines with $d$ tape symbols. As for the number $d$, it is well known that any algorithm can be realized on Turing machines with $d=2$, using a sufficient number of state symbols and with appropriate coding for inputs and outputs. As the number of tape symbols does not at all influence the properties of the theory we are going to describe, we shall not aspire to limit ourselves to this minimum value of $d=2$. Rather, Turing machines with $d=5$ will be used for implementing our algorithms. For greater clarity, these five tape symbols $a_{0}, a_{1}, a_{2}, a_{3}, a_{4}$ will be denoted, respectively, by $0,1, B, 0^{\prime}, 1^{\prime}$.

In the paper, we use some special class $S p e c S T \subseteq S T_{5}$ of starting situations which includes all situations of the form shown in Fig. 3.2. Such a situation

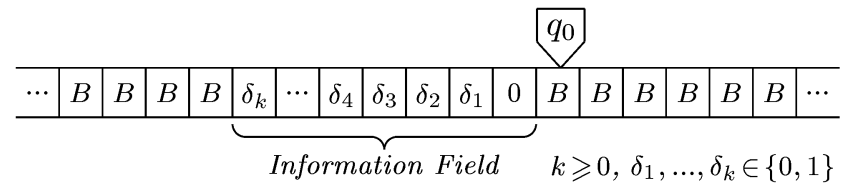

Fig. 3.2. Special Start position of a Turing machine $\mathcal{M}$

includes an information field in the form of a finite sequence of 0's and 1's, while the other tape cells to the left and right of the field should be filled with blanks. The very right symbol of the information field must be 0 , and the starting position of head must be the blank symbol just to the right of the rightmost symbol of the information field.

One more important comment applies to halting. In the case when the configuration $a_{i} q_{j}$ does not correspond to any command of $\mathcal{M}$, we say that this machine has a quasi-command of the form $a_{i} q_{j} \rightarrow$ stop, and the action of this quasi-command is to enter the halting configuration. We shall consider $\mathcal{M}$ as the set of its commands and quasi-commands. For definiteness, we write $\langle i, j, m, t, L\rangle \in \mathcal{M}$ if the command $a_{i} q_{j} \rightarrow a_{m} q_{t} L$ belongs to $\mathcal{M}$, we write $\langle i, j, m, t, R\rangle \in \mathcal{M}$ if the command $a_{i} q_{j} \rightarrow a_{m} q_{t} R$ belongs to $\mathcal{M}$, and we write $\langle i, j$, stop $\rangle \in \mathcal{M}$ if the quasi-command $a_{i} q_{j} \rightarrow$ stop belongs to $\mathcal{M}$. Thus, for any $i<d$ and $j<e$, a machine should have exactly one command or quasi-command which is applicable to the situation $a_{i} q_{j}$.

Let $\mathcal{M}$ be a Turing machine as described above, and fix some initial configuration $\mathcal{S} \in S T_{5}$. To illustrate these concepts, we consider as an example the following list of commands:

$$
\begin{aligned}
& 1 q_{0} \rightarrow 1 q_{0} L \\
& B q_{0} \rightarrow 0 q_{1} R \\
& 1 q_{1} \rightarrow 1 q_{1} R \\
& B q_{1} \rightarrow 0 q_{2} L \\
& 1 q_{2} \rightarrow \text { stop }
\end{aligned}
$$

and we take the initial configuration as shown in Fig. 3.3 (a). It can be verified 
that after starting, the machine works a finite number of steps before halting in the configuration shown in Fig. 3.3 (b).

Now we construct the following natural model which will represent a computation on $\mathcal{M}$ beginning from $\mathcal{S}$. We consider the upper half of the real plane $\mathbb{R} \times \mathbb{R}$, on which we shall draw some infinite figures. First we draw the sequence of starting cells of the tape as a horizontal row in which adjacent cells are connected by line segments (see the bottom row in Fig. 3.4). We write in each cell the corresponding tape symbol from the initial configuration $\mathcal{S}$.

In the cell initially scanned by the head, we write the initial state of the machine (which under our conventions is $q_{0}$ ). We call this row the zero row. Above the zero row, we draw the first row, arranging its cells strictly above the cells in the zero row. We continue this process to construct the second row and so on. Vertically adjacent cells are connected by line segments, and as a result we obtain the picture shown in Fig. 3.4.

(a)

\begin{tabular}{c|c|c|c|c|c|c|c|c|c|c}
$\cdots$ & $B$ & $B$ & $B$ & $B$ & 1 & 1 & $B$ & $B$ & $B$ & $B$ \\
\hline
\end{tabular}

(b)

\begin{tabular}{l|l|l|l|l|l|l|l|l|l|l|l}
\hline$\cdots$ & $B$ & $B$ & $B$ & 0 & 1 & 1 & 0 & $B$ & $B$ & $B$ & $\cdots$ \\
\hline
\end{tabular}

Fig. 3.3. Start and Halt States of machine $\mathcal{M}$

Each row of cells represents the tape of a Turing machine at one stage of a computation, and vertically adjacent cells represent the same cell at consecutive stages. Now we begin filling the tape cells with information. The cell in which the current state is written also indicates the position of the head. We fill in the first row as follows. Each cell not directly above the cell scanned by the head is marked with the symbol that was present in the cell below. The cell directly above the state symbol $q_{0}$ is filled according to the action of the appropriate command of the Turing machine. The same command defines the position of the head and the corresponding state of the machine. In our example, the configuration $1 q_{0}$ appears in the zero row, therefore the command $1 q_{0} \rightarrow 1 q_{0} R$ applies. The cell in the first row directly above this position is marked with the symbol 1 and the movement of the head is indicated by marking the cell to the right with the state $q_{0}$. We continue to label the rows in this manner. When all the rows are filled, the figure is complete. For clarity, the position of the head is marked by a dashed line.

We must also consider the case when a halting configuration is reached (of course, this situation does not arise in all computations). In the case of a halting configuration, as in the sixth row of Fig. 3.4, we discontinue the head sequence, and in subsequent rows, the position of head and state of the machine are not underlined. The last row in a computation containing the head represents the configuration on the tape after the machine has stopped (see Fig. 3.4.). We could extract the output of the Turing machine (for example, the number of ones on the tape) from this row. 
In summary, we obtain a figure on which the initial configuration of the machine as well as all intermediate configurations and the halting configuration (if it exists) are represented. To make the figure more informative, to the right of each row we note the command which is applicable to the situation indicated on the tape, see Fig. 3.4. The command $a_{i} q_{j} \rightarrow$ stop (noted at most once) specifies the halting stage of the machine.

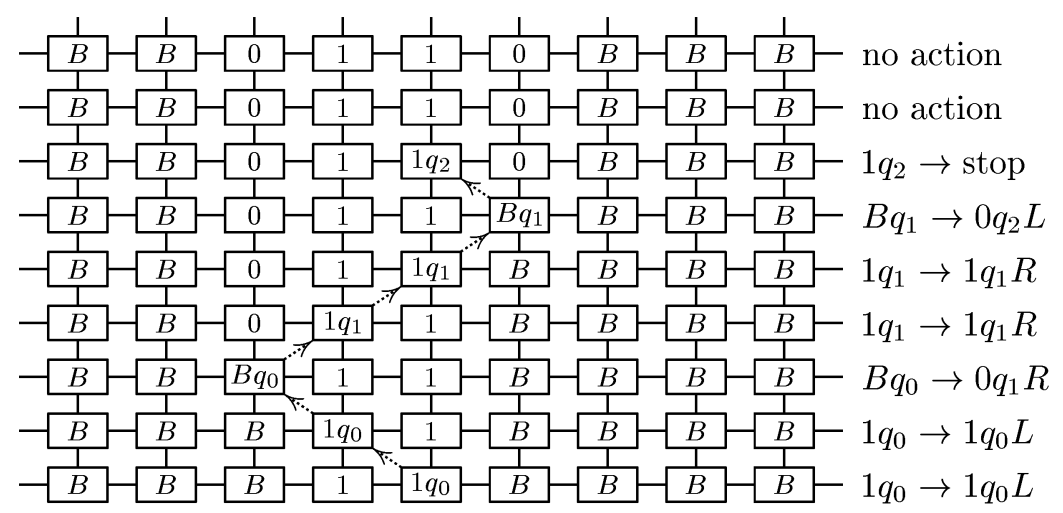

Fig. 3.4. Computation unfolded in time on Turing machine $\mathcal{M}$

It is possible to abstract from the inessential details of the description above and to give a strictly formal definition for a computation unfolded in time on a Turing machine. It is natural to present the picture described above as a model $\mathfrak{N}$ of the following signature

$$
\sigma=\left\{S^{2}, N^{2}, O, J, A_{0}, A_{1}, \ldots, A_{d-1}, Q, Q_{0}, Q_{1}, \ldots, Q_{e-1}\right\}
$$

where $d$ and $e$ are parameters denoting the number of tape symbols and the number of states of the machine.

The universe of the model is $|\mathfrak{N}|=\mathbb{Z} \times \mathbb{N}$. The elements of this set represent integer grid points on the upper half of the plane. The element $(0,0) \in \mathfrak{N}$ corresponds to the cell scanned by the head at the initial stage. The predicates of the signature are defined as follows:

$$
\begin{aligned}
S(x, y) & \Leftrightarrow y \text { is the right neighbor of } x, \\
N(x, y) & \Leftrightarrow y \text { is directly above } x, \\
A_{i}(x) & \Leftrightarrow \text { the symbol } a_{i} \text { is in cell } x, \\
Q(x) & \Leftrightarrow \text { the head scans cell } x, \\
Q_{j}(x) & \left.\Leftrightarrow Q(x) \& \text { (the machine is in state } q_{j}\right), \\
J(x) & \Leftrightarrow x \text { is in the bottom row, } \\
O(x) & \Leftrightarrow J(x) \& Q(x) .
\end{aligned}
$$


It is possible to give a strictly formal (analytical) definition of the model $\mathfrak{N}$, determined by the initial configuration and the program of the machine $\mathcal{M}$.

The model $\mathfrak{N}$ of signature $\sigma$ defined above is called a computation unfolded in time of the machine $\mathcal{M}$ beginning from the initial configuration $\mathcal{S}$. More briefly, we call this an $\mathcal{S}$-computation on $\mathcal{M}$ and denote it by

$$
\mathfrak{N}=\operatorname{Compu}(\mathcal{M}, \mathcal{S}) .
$$

This model will be as in Figure 3.4, using the terms as marked.

Consider a computation unfolded in time on a Turing machine starting from the initial configuration $\mathcal{S}$. We assume that the $\mathcal{S}$-computation on $\mathcal{M}$ halts at point $h$. Label by $t$ the point located one row above $h$. Drawing two diagonal lines $l_{0}$ and $l_{1}$ from $t$, we obtain a triangle with the top corner $t$, two sides $l_{0}$ and $l_{1}$, and a segment of the zero row as its base. We "clip" this triangle from the semiplane and consider it separately. It is called the triangular clipping of the computation or, more briefly, the triangle of computation.

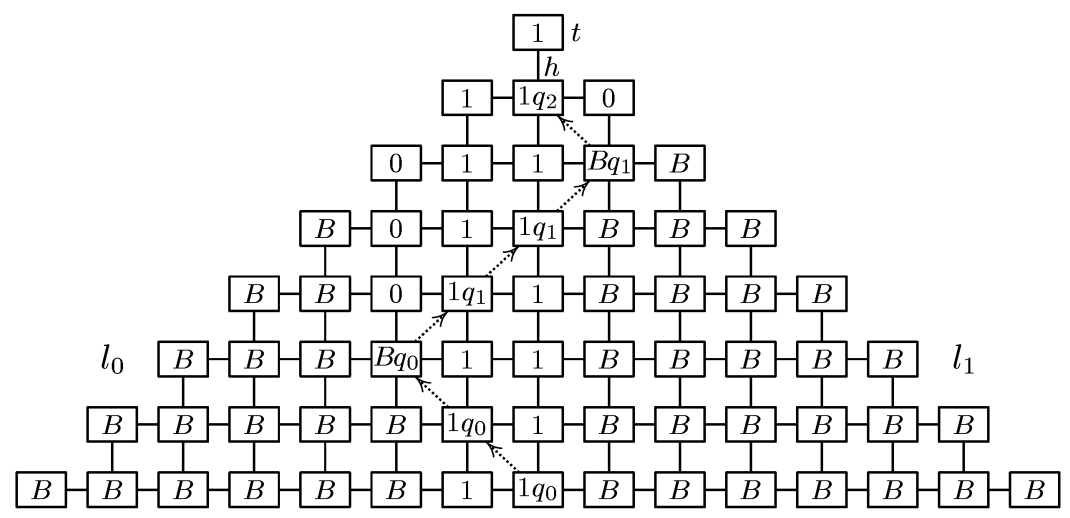

Fig. 3.5. Triangle of Computation on a Turing machine

The triangle of computation does not exist (is undefined) if the Turing machine does not halt. Applied to Fig. 3.4, this procedure yields the triangle shown in Fig. 3.5.

\section{Design of a finitely axiomatizable theory}

In this section, we represent the overall schema of the proof of our Main Theorem 1.5, showing all the steps of the proof in their logical sequence. Some of these steps are proved here, while others are just quoted and proved in the later sections of the paper. 
We suppose that some fixed $\Sigma_{2}^{0}$ Boolean algebra $(\mathcal{B}, \nu)$ is given. Our main goal is to construct a sentence $\Omega=\Omega(\mathcal{B}, \nu)$ of a finite rich signature $\sigma_{0}$ (which includes the symbols in (3.3) and is formally defined in Section 6) satisfying the condition

$$
(\mathcal{B}, \nu) \cong\left(\mathcal{L}^{*}(\operatorname{ModFin}(\Omega)), \gamma\right),
$$

where $\mathcal{L}^{*}(H)$ for a theory $H$ denotes the quotient algebra $\mathcal{L}(H) / \mathcal{F}(\mathcal{L}(H))$ modulo the Fréchet ideal $\mathcal{F}(\mathcal{L}(H))$, while $\gamma$ is a fixed Gödel enumeration of $S L\left(\sigma_{0}\right)$.

Hereafter, we use the notation $T$ for the finitely axiomatizable theory generated by the sentence $\Omega$ as an axiom, i.e., this theory is defined by $T=[\Omega]$.

Without loss of generality, we will assume the algebra $\mathcal{B}$ to be nontrivial, i.e., with $0 \neq 1$. We can also suppose that its numeration $\nu$ satisfies the conditions:

$$
\nu^{-1}(0) \text { is infinite, and } \nu^{-1}(1) \text { is infinite. }
$$

There are recursive functions $u(x, y), v(x, y), w(x)$ and a recursive relation $P(y, z, t)$ which represent the given $\Sigma_{2}^{0}$ Boolean algebra, i.e., satisfying, for the operations, the conditions

$$
\begin{aligned}
& \nu(k) \cup \nu(n)=\nu(u(k, n)), \\
& \nu(k) \cap \nu(n)=\nu(v(k, n)), \\
& -\nu(k)=\nu(w(k)),
\end{aligned}
$$

as well as, for the recursive relation $P(y, z, t)$, the condition

$$
\begin{aligned}
\nu(0)^{\varepsilon_{0}} \cap \nu(1)^{\varepsilon_{1}} \cap \ldots \cap \nu(m-1)^{\varepsilon_{m-1}}=0 \Leftrightarrow \\
(\exists y)(\forall z) P\left(y, z, \operatorname{Nom}\left\langle\varepsilon_{0}, \varepsilon_{1}, \ldots, \varepsilon_{m-1}\right\rangle\right),
\end{aligned}
$$

for any $k, n, m \in \mathbb{N}$ and $\varepsilon_{0}, \varepsilon_{1}, \ldots, \varepsilon_{m-1} \in\{0,1\}$. Here Nom $\left\langle\varepsilon_{0}, \varepsilon_{1}, \ldots, \varepsilon_{m-1}\right\rangle$ denotes the Gödel number of the sequence $\left\langle\varepsilon_{0}, \varepsilon_{1}, \ldots, \varepsilon_{m-1}\right\rangle$ as defined in Section 2. The case $m=0$ is allowed as well, so the tuple in (4.4) can be empty. In this case, we have $\forall y \exists z \neg P(y, z, \operatorname{Nom}\langle\rangle)$.

The finitely axiomatizable theory $T$ includes the computation of some Turing machine $\mathcal{M}$ subject to the description given in Section 3. We use a machine $\mathcal{M}$ with 5 tape symbols denoted by $0,1, B, 0^{\prime}, 1^{\prime}$. The machine $\mathcal{M}$ starts from the tape position depicted in Fig. 4.1, where $\left\langle\varepsilon_{0}, \varepsilon_{1}, \ldots, \varepsilon_{m-1}\right\rangle$ is a finite sequence of zeros and ones. For simplicity, this sequence is pictured (from now on) as a sequence writh $m-1$ i a ac lon s. on sol

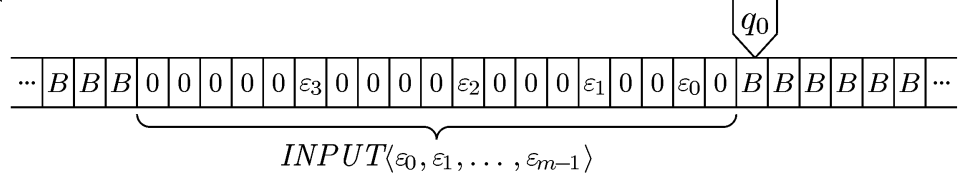

Fig. 4.1. Start state of Turing machine $\mathcal{M}$ 
The starting information is defined by a finite sequence of zeros and ones, $\varepsilon=\left\langle\varepsilon_{0}, \varepsilon_{1}, \ldots, \varepsilon_{m-1}\right\rangle$ with the bit cells $\varepsilon_{i}$ separated by increasing intervals of zeros. The information field $\operatorname{INPUT}\left\langle\varepsilon_{0}, \varepsilon_{1}, \ldots, \varepsilon_{m-1}\right\rangle$ ends with a sequence of zeros followed by blanks. Also, the entire right side of the tape is filled with blanks, including the cell scanned by the head. For the information field $\operatorname{INPUT}\left\langle\varepsilon_{0}, \varepsilon_{1}, \ldots, \varepsilon_{m-1}\right\rangle$, we denote by weight(INPUT) the number $m$ of bit information cells $\varepsilon_{i}$ contained in the field.

$\operatorname{INPUT}\left\langle\varepsilon_{0}, \varepsilon_{1}, \ldots, \varepsilon_{m-1}\right\rangle$ will be used not only to refer to an information field of the tape but also to refer to the starting situation as a whole on the tape as shown in Fig. 4.1. (The context will make clear which notation is meant.)

To establish a plan for the finitely axiomatizable theory $T$, we first give a general description of the algorithm used for this theory. This will allow us to give a brief sketch of the theory as a whole, while the exact algorithm itself will be constructed later.

The algorithm is described by the following program (realized later as the computation on a Turing machine $\mathcal{M}$ ):

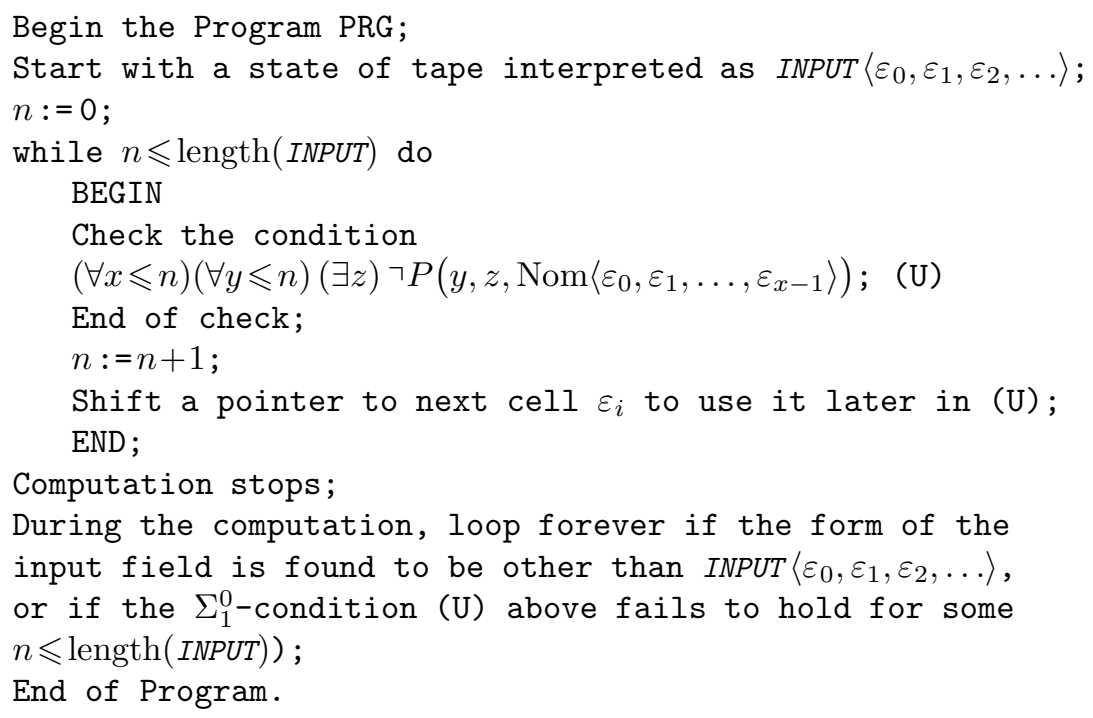

The $P R G$ instruction to "loop forever if the form of input information is found to be other than $\operatorname{INPUT}\left\langle\varepsilon_{0}, \varepsilon_{1}, \varepsilon_{2}, \ldots\right\rangle$ " is formally specified by the condition:

$$
\begin{aligned}
& (\forall \mathcal{S} \in \operatorname{SpecST})\left[\mathcal{S} \text { does not have form INPUT }\langle\varepsilon\rangle \text { for some } \varepsilon \in 2^{<\omega}\right. \\
& \Rightarrow \mathcal{S} \text {-computation on machine } \mathcal{M} \text { does not stop }] \text {. }
\end{aligned}
$$

In addition, the conditions under which $P R G$ reaches a halt state if started with 
correct information, are formalized by the condition

$$
\begin{aligned}
& (\forall m \in \mathbb{N})\left(\forall \varepsilon_{0}, \ldots, \varepsilon_{m-1} \in\{0,1\}\right) \\
& {\left[I N P U T\left\langle\varepsilon_{0}, \ldots, \varepsilon_{m-1}\right\rangle\right. \text {-computation reaches a halt state }} \\
& \left.\Leftrightarrow(\forall n \leqslant m)(\forall x \leqslant n)(\forall y \leqslant n)(\exists z)\urcorner P\left(y, z, \operatorname{Nom}\left\langle\varepsilon_{0}, \ldots, \varepsilon_{x-1}\right\rangle\right)\right] .
\end{aligned}
$$

The description of $P R G$ is finished.

According to the specification of the algorithm $P R G$, the machine gradually reads the input information on the tape, checking that the starting information has the form $\operatorname{INPUT}\left\langle\varepsilon_{0}, \varepsilon_{1}, \ldots, \varepsilon_{n-1}, \ldots\right\rangle$, that is, has the correct number of zeros between its bit cells $\varepsilon_{i}$, while in the (real) bit cells, any of the symbols 0,1 , and $B$ may occur. When a symbol read in a cell (where the next bit $\varepsilon_{i}$ should be taken) turns to be the blank symbol $B$, then the field INPUT is assumed to be finished, and this (and only this) situation leads to the final part of computation and then to the halting state of the algorithm. Note that to reach such a blank symbol, it is necessary that all conditions enumerated in the cycle for different values of $n, x$ and $y$ hold (if the $\exists$ block of the condition fails, it will lead to a computation which "non-stops", and therefore we will not leave this block at all).

A detailed description of the Turing machine $\mathcal{M}$ implementing the algorithm $P R G$ is presented in Section 5.

For the Turing machine $\mathcal{M}$, we denote by $\operatorname{Hlt}(\mathcal{M})$ the set of all sequences $\left\langle\varepsilon_{0}, \varepsilon_{1}, \ldots, \varepsilon_{m-1}\right\rangle \in 2^{<\omega}$ such that the machine $\mathcal{M}$ stops after it begins its work from the start condition $\operatorname{INPUT}\left\langle\varepsilon_{0}, \varepsilon_{1}, \ldots, \varepsilon_{m-1}\right\rangle$. That is,

$$
\operatorname{Hlt}(\mathcal{M})=\left\{\left\langle\varepsilon_{0}, \ldots, \varepsilon_{m-1}\right\rangle \mid \mathcal{M} \text { stops when started with } \operatorname{INPUT}\left\langle\varepsilon_{0}, \ldots, \varepsilon_{m-1}\right\rangle\right\} \text {. }
$$

From the description (4.6) of the algorithm $P R G$, we have

$$
\begin{aligned}
& \operatorname{Hlt}(\mathcal{M})= \\
& \left.\left\{\left\langle\varepsilon_{0}, \varepsilon_{1}, \ldots, \varepsilon_{m-1}\right\rangle \mid(\forall x \leqslant m)(\forall y \leqslant m)(\exists z)\right\urcorner P\left(y, z, \operatorname{Nom}\left\langle\varepsilon_{0}, \ldots, \varepsilon_{x-1}\right\rangle\right)\right\},
\end{aligned}
$$

and as a simple consequence of (4.7), we obtain the property:

$$
\left(\forall \varepsilon, \varepsilon^{\prime} \in 2^{<\omega}\right)\left[\varepsilon \preccurlyeq \varepsilon^{\prime} \& \varepsilon^{\prime} \in \operatorname{Hlt}(\mathcal{M}) \Rightarrow \varepsilon \in \operatorname{Hlt}(\mathcal{M})\right] .
$$

Denote by Cons the set of sequences $\left\langle\varepsilon_{0}, \varepsilon_{1}, \ldots, \varepsilon_{m-1}\right\rangle \in 2^{<\omega}$ for which the negation of the left-hand side of condition (4.4) is true. In particular, we have

$$
\begin{aligned}
\text { Cons } & =\left\{\left\langle\varepsilon_{0}, \varepsilon_{1}, \ldots, \varepsilon_{m-1}\right\rangle \mid \nu(0)^{\varepsilon_{0}} \cap \nu(1)^{\varepsilon_{1}} \cap \ldots \cap \nu(k)^{\varepsilon_{m-1}} \neq 0\right\} \\
& \left.=\left\{\left\langle\varepsilon_{0}, \varepsilon_{1}, \ldots, \varepsilon_{m-1}\right\rangle \mid(\forall y)(\exists z)\right\urcorner P\left(y, z, \operatorname{Nom}\left\langle\varepsilon_{0}, \varepsilon_{1}, \ldots, \varepsilon_{m-1}\right\rangle\right)\right\} .
\end{aligned}
$$


Using the conditions $0 \neq 1,(4.7),(4.9)$ and (4.10), we obtain that the following properties hold:

$$
\begin{aligned}
& \langle\rangle \in \text { Cons, } \\
& (\forall \varepsilon)[\varepsilon \in \text { Cons } \Leftrightarrow \varepsilon 0 \in \text { Cons } \vee \varepsilon 1 \in \text { Cons }], \\
& \left(\forall \varepsilon, \varepsilon^{\prime} \in 2^{<\omega}\right)\left[\varepsilon \preccurlyeq \varepsilon^{\prime} \& \varepsilon^{\prime} \in \text { Cons } \Rightarrow \varepsilon \in \text { Cons }\right], \\
& \text { Cons } \subseteq \operatorname{Hlt}(\mathcal{M}), \\
& (\forall \varepsilon)\left[\varepsilon \notin \text { Cons } \Leftrightarrow(\exists k)\left(\forall \varepsilon^{\prime} \succ \varepsilon\right)\left(\left|\varepsilon^{\prime}\right|>|\varepsilon|+k \Rightarrow \varepsilon^{\prime} \notin \operatorname{Hlt}(\mathcal{M})\right)\right], \\
& \left(\forall \varepsilon^{*} \in 2^{\omega}\right)\left\{\left(\forall \varepsilon \prec \varepsilon^{*}\right)[\varepsilon \in \text { Cons }] \Leftrightarrow\left(\forall \varepsilon \prec \varepsilon^{*}\right)[\varepsilon \in \operatorname{Hlt}(\mathcal{M})]\right\} .
\end{aligned}
$$

In Section 6 (after the Turing machine $\mathcal{M}$ is described), we construct a finitely axiomatizable theory $T=[\Omega]$ of a finite signature $\sigma_{0}$ including the predicates (3.3) (and a few others), whose models represent the computations of machine $\mathcal{M}$ in the sense described in Section 3. By our construction, for each finite sequence $\left\langle\varepsilon_{0}, \varepsilon_{1}, \ldots, \varepsilon_{m-1}\right\rangle \in \operatorname{Hlt}(\mathcal{M})$, the theory $T$ will have a finite model $\mathfrak{M}\left\langle\varepsilon_{0}, \varepsilon_{1}, \ldots, \varepsilon_{m-1}\right\rangle$ representing the triangle of computation beginning with the starting condition $\operatorname{INPUT}\left\langle\varepsilon_{0}, \varepsilon_{1}, \ldots, \varepsilon_{m-1}\right\rangle$. Our construction will ensure that the models of the theory $T$ satisfy the following key condition:

$$
\operatorname{ModFin}(T)=\operatorname{Mod}(T) \cap M_{\text {fin }}=\{\mathfrak{M}\langle\varepsilon\rangle \mid \varepsilon \in \operatorname{Hlt}(\mathcal{M})\} .
$$

After the algorithm $\mathcal{M}$ is completely described and the theory $T$ is constructed for which the condition (4.17) is established, we turn to the final (technically complicated) part of the proof, in which it is shown that the obtained theory $T$ satisfies the needed property (4.1).

The final part of the proof consists of the following main steps.

An infinite sequence of zeros and ones $\varepsilon=\left\langle\varepsilon_{0}, \varepsilon_{1}, \varepsilon_{2}, \ldots\right\rangle$ is called consistent if for any $m>0$, the model $\mathfrak{M}\left\langle\varepsilon_{0}, \varepsilon_{1}, \ldots, \varepsilon_{m-1}\right\rangle$ exists. By Cons* we denote the set of all infinite consistent sequences of zeros and ones, i.e.,

$$
\text { Cons }^{*}=\left\{\varepsilon \in 2^{\omega} \mid(\forall k \in \mathbb{N})[\varepsilon\lceil k \in \operatorname{Hlt}(\mathcal{M})]\} .\right.
$$

By virtue of the property (4.16), we also have the following representation

$$
\varepsilon \in \text { Cons }^{*} \Leftrightarrow \varepsilon \in 2^{\omega} \&(\forall k \in \mathbb{N})[\varepsilon\lceil k \in \text { Cons }] .
$$

In particular, we have Cons ${ }^{*} \neq \varnothing$ as a simple consequence of (4.19) together with (4.11) and (4.12).

We will need the following technical statement in Section 9. 
Lemma 4.1. There is a function $f: \mathbb{N} \rightarrow \mathbb{N}$ which satisfies the following conditions:

$$
\begin{aligned}
& f(x) \text { is an increasing function (not necessarily strictly), } \\
& \lim _{n \rightarrow \infty} f(n)=\infty, \\
& \left(\forall \varepsilon \in \text { Cons }^{*}\right)(\forall n)\left[\text { Card }\left\{i \mid i<n \& \varepsilon_{i}=0\right\} \geqslant f(n)\right] \\
& \left(\forall \varepsilon \in \text { Cons }^{*}\right)(\forall n)\left[\text { Card }\left\{i \mid i<n \& \varepsilon_{i}=1\right\} \geqslant f(n)\right] .
\end{aligned}
$$

Proof. Consider the function $f(x)$ defined by the following rule

$$
\begin{aligned}
& f(n)=\min \left\{f_{0}(n), f_{1}(n)\right\}, \text { where } \\
& f_{0}(n)=\min _{\varepsilon \in \text { Cons }^{*}} \operatorname{Card}\left\{i \mid i<n \& \varepsilon_{i}=0\right\}, \\
& f_{1}(n)=\min _{\varepsilon \in \text { Cons }^{*}} \operatorname{Card}\left\{i \mid i<n \& \varepsilon_{i}=1\right\} .
\end{aligned}
$$

It follows directly from the definitions of $f_{0}(n)$ and $f_{1}(n)$ that both are increasing (possibly not strictly). Therefore, the function $f(n)$ defined by (4.24) is increasing as well. The condition (4.2) together with (4.9) and (4.19) provide that $\lim _{n \rightarrow \infty} f_{0}(n)=\infty$ and $\lim _{n \rightarrow \infty} f_{1}(n)=\infty$. Therefore, the three conditions (4.21)-(4.23) directly follow from the definition (4.24) of function $f(n)$.

For models $\mathfrak{M}$ and $\mathfrak{N}$, we write $\mathfrak{M} \equiv{ }_{s} \mathfrak{N}$ if they are equivalent with respect to sentences with $\leqslant s$ quantifiers (in Skolem normal form). In Sections 7-11 we prove that the models of the theory $T$ satisfy the following condition:

$$
\begin{aligned}
& (\forall s>0)\left(\forall \varepsilon^{*} \in \mathrm{Cons}^{*}\right)(\exists k>0) \\
& \left(\forall \varepsilon^{\prime}, \varepsilon^{\prime \prime} \in \operatorname{Hlt}(\mathcal{M})\right)\left[\varepsilon^{*}\left|k \preccurlyeq \varepsilon^{\prime} \& \varepsilon^{*}\right| k \preccurlyeq \varepsilon^{\prime \prime} \Rightarrow \mathfrak{M}\left\langle\varepsilon^{\prime}\right\rangle \equiv_{s} \mathfrak{M}\left\langle\varepsilon^{\prime \prime}\right\rangle\right] .
\end{aligned}
$$

From this, we can prove the following stronger statement.

Lemma 4.2. The following assertion holds:

$$
\begin{aligned}
& (\forall s>0)(\exists k>0)\left(\forall \varepsilon^{*} \in \mathrm{Cons}^{*}\right) \\
& \left(\forall \varepsilon^{\prime}, \varepsilon^{\prime \prime} \in \operatorname{Hlt}(\mathcal{M})\right)\left[\varepsilon^{*}\left|k \preccurlyeq \varepsilon^{\prime} \& \varepsilon^{*}\right| k \preccurlyeq \varepsilon^{\prime \prime} \Rightarrow \mathfrak{M}\left\langle\varepsilon^{\prime}\right\rangle \equiv_{s} \mathfrak{M}\left\langle\varepsilon^{\prime \prime}\right\rangle\right] .
\end{aligned}
$$

Proof. We deduce (4.26) as a consequence of (4.25). Let a value of $s>0$ be given. Consider the set $\mathcal{D}$ of all tuples $\varepsilon \in 2^{<\omega}$ satisfying the condition

$$
\left(\exists \varepsilon^{\prime}, \varepsilon^{\prime \prime} \in \operatorname{Hlt}(\mathcal{M})\right)\left[\varepsilon \preccurlyeq \varepsilon^{\prime} \& \varepsilon \preccurlyeq \varepsilon^{\prime \prime} \& \mathfrak{M}\left\langle\varepsilon^{\prime}\right\rangle \not \equiv_{s} \mathfrak{M}\left\langle\varepsilon^{\prime \prime}\right\rangle\right] .
$$

Obviously, we have $\left(\forall \varepsilon, \varepsilon_{1} \in 2^{<\omega}\right)\left[\varepsilon \preccurlyeq \varepsilon_{1} \& \varepsilon_{1} \in \mathcal{D} \Rightarrow \varepsilon \in \mathcal{D}\right]$, i.e., the set $\mathcal{D}$ is a subtree of binary branching tree $2^{<\omega}$. Therefore, it cannot be infinite, because otherwise a tuple $\varepsilon^{*}$ of length $\omega$ could be found such that each of its 
initial segments belongs to $\mathcal{D}$. But obviously this sequence contradicts statement (4.25). Thus, the set $\mathcal{D}$ is finite. Therefore it is possible to find a natural number $k$ which exceeds the lengths of all tuples from $\mathcal{D}$. This value of $k$ establishes (4.26).

Consider the following series of sentences of the theory $T$, which are expressible via predicates (3.3) of the signature $\sigma_{0}$ from (3.4):

$$
\begin{aligned}
& \Delta_{k}={ }_{d f}\left[\text { the } \frac{1}{2}(k+1)(k+4) \text {-th } S \text {-predecessor of } O \text { satisfies } A_{1}(x)\right], \\
& \Theta_{k}={ }_{d f}\left[\text { the } \frac{1}{2}(k+1)(k+4) \text {-th } S \text {-predecessor of } O \text { satisfies } A_{0}(x) \vee A_{1}(x)\right] .
\end{aligned}
$$

These sentences speak about the starting information of the Turing machine. Namely, $\Delta_{k}$ says that the input sequence $\varepsilon=\left\langle\varepsilon_{0}, \varepsilon_{1}, \ldots\right\rangle$ satisfies $\varepsilon_{k}=1$, while $\Theta_{k}$ says that $\varepsilon_{k} \in\{0,1\}$, i.e., the length of the input sequence is $k+1$ or more (there is no blank symbol $B$ in the corresponding cell of the input sequence). In particular, we have $\Theta_{j} \rightarrow \Theta_{j-1}$ for all $j>0$ in all finite models of $T$. One can see that both sentences $\Delta_{k}$ and $\Theta_{k}$ can be constructed effectively in $k \in \mathbb{N}$.

Now, we prove the following important property:

Lemma 4.3. The set of sentences $\Delta_{i}, \Theta_{i}, i \in \mathbb{N}$, is a generating set for the Lindenbaum algebra $\mathcal{L}(\operatorname{ModFin}(T))$.

Proof. Let $\Phi$ be a sentence of the theory $T$, and let $s$ be the number of quantifiers of $\Phi$ after it is reduced to Skolem normal form. In particular, for the sentence $\Phi$ and for any models $\mathfrak{M}, \mathfrak{N}$ of signature $\sigma_{0}$, we have:

$$
\mathfrak{M} \equiv{ }_{s} \mathfrak{N} \Rightarrow \mathfrak{M} \models \Phi \Leftrightarrow \mathfrak{N} \models \Phi .
$$

Take $k>0$ which makes the statement (4.26) true for this $s$. Consider the following two sets of tuples:

$$
\begin{aligned}
& L_{\Phi}=\{\varepsilon \in \operatorname{Hlt}(\mathcal{M})|| \varepsilon \mid<k \& \mathfrak{M}\langle\varepsilon\rangle \models \Phi\}, \\
& M_{\Phi}=\{\varepsilon \in \operatorname{Hlt}(\mathcal{M})|| \varepsilon \mid=k \& \mathfrak{M}\langle\varepsilon\rangle \models \Phi\} .
\end{aligned}
$$

For a sequence $\varepsilon \in 2^{<\omega}, \varepsilon=\left\langle\varepsilon_{0}, \varepsilon_{1}, \ldots, \varepsilon_{j-1}\right\rangle$, we denote by $I N^{*}\langle\varepsilon\rangle$ and $I N\langle\varepsilon\rangle$ the following formulas of the signature $\sigma_{0}$ :

$$
\begin{aligned}
& \left.I N^{*}\langle\varepsilon\rangle=\Delta_{0}^{\varepsilon_{0}} \& \Delta_{1}^{\varepsilon_{1}} \& \ldots \& \Delta_{j-1}^{\varepsilon_{j-1}} \& \Theta_{j-1} \&\right\urcorner \Theta_{j}, \\
& I N\langle\varepsilon\rangle=\Delta_{0}^{\varepsilon_{0}} \& \Delta_{1}^{\varepsilon_{1}} \& \ldots \& \Delta_{j-1}^{\varepsilon_{j}-1} \& \Theta_{j-1} .
\end{aligned}
$$

One can see, $I N\langle\varepsilon\rangle$ is true in all models $\mathfrak{M}\left\langle\varepsilon^{\prime}\right\rangle$ satisfying $\varepsilon \preccurlyeq \varepsilon^{\prime}$, while $I N^{*}\langle\varepsilon\rangle$ is true only in the finite model $\mathfrak{M}\langle\varepsilon\rangle$ of the class (4.17).

Consider the following sentence of the signature $\sigma_{0}$ :

$$
\Psi=\bigvee_{\varepsilon \in L_{\Phi}} I N^{*}\langle\varepsilon\rangle \vee \bigvee_{\varepsilon \in M_{\Phi}} I N\langle\varepsilon\rangle .
$$


Using the properties (4.17) and (4.26) for the given $s$ and $k$, and the fact that the truth value of $\Phi$ is preserved under $\equiv_{s}$ on models, one can check that the sentence $\Phi \leftrightarrow \Psi$ is true in each finite model $\mathfrak{M}$ of the theory $T$. This completes the proof of Lemma 4.3 because the formula (4.27) is obviously a Boolean expression over formulas $\Delta_{i}$ and $\Theta_{i}, i \in \mathbb{N}$.

Now, we turn to the quotient modulo the Fréchet ideal.

Lemma 4.4. The set of sentences $\Delta_{i}, i \in \mathbb{N}$, is a generating set for the quotient of the Lindenbaum algebra

$$
\mathcal{L}^{*}(\operatorname{ModFin}(T))=\mathcal{L}(\operatorname{ModFin}(T)) / \mathcal{F}
$$

modulo the Fréchet ideal.

Proof. By Lemma 4.3, the set of sentences $\Delta_{i}, \Theta_{i}, i \in \mathbb{N}$, is a generating set for Lindenbaum algebra $\mathcal{L}(\operatorname{ModFin}(T))$, and therefore it is a generating set for its quotient modulo the Fréchet ideal. But, any formula $\Theta_{i}$ is equal to 1 modulo the ideal $\mathcal{F}$, because $\urcorner \Theta_{i}$ is obviously true in a finite number of finite models of the class (4.17). Therefore, the set $\Delta_{i}, i \in \mathbb{N}$, is a generating set for the quotient.

Now, we are in a position to prove our main statement (4.1).

Let $u(x, y), v(x, y)$, and $w(x)$ be general recursive functions which satisfy (4.3). If $\Phi$ is a sentence of signature $\sigma_{0}$, we let $[\Phi]^{*}$ denote the equivalence class which is generated by this formula in the quotient algebra $\mathcal{L}(\operatorname{ModFin}(T)) / \mathcal{F}$ modulo the Fréchet ideal $\mathcal{F}$.

We define the mapping $\lambda$ from $\mathcal{B}$ into $\mathcal{L}(\operatorname{ModFin}(T)) / \mathcal{F}$ by the following rule:

$$
\lambda(\nu(n))=\left[\Delta_{n}\right]^{*}, n \in \mathbb{N} .
$$

The fact that this map achieves our goal is verified in the next lemma.

Lemma 4.5. The map $\lambda$ is a constructive isomorphism between the numerated Boolean algebras $(\mathcal{B}, \nu)$ and $(\mathcal{L}(\operatorname{ModFin}(T)) / \mathcal{F}, \gamma / \mathcal{F})$.

Proof. By (4.28), the mapping $\lambda$ is defined on all elements of $\mathcal{B}$.

We first check that $\lambda$ is well defined.

Let $m, n$ be such that $\nu(n)=\nu(m)$, and let $p=\max \{m, n\}+1$. Then for any sequence $\varepsilon=\left\langle\varepsilon_{0}, \varepsilon_{1}, \ldots\right\rangle$, for which

$$
\varepsilon \in 2^{<\omega},|\varepsilon| \geqslant p \text {, and }\left(\varepsilon_{m}, \varepsilon_{n}\right) \in\{(1,0),(0,1)\},
$$

we have $\varepsilon \notin$ Cons by (4.9). There are only finitely many sequences $\varepsilon$ of the form (4.29) with $|\varepsilon|=p$, while all other sequences of the class (4.29) are their extensions. Then, by (4.15), we obtain that there are only finitely many sequences $\varepsilon \in \operatorname{Hlt}(\mathcal{M})$ for which condition (4.29) holds. Also, there can be only 
finitely many sequences $\varepsilon \in \operatorname{Hlt}(\mathcal{M})$ satisfying $|\varepsilon|<p$. Therefore, for any $\alpha, \beta$ satisfying

$$
(\alpha, \beta) \in\{(0,1),(1,0)\},
$$

the sentence $\Delta_{m}^{\alpha} \& \Delta_{n}^{\beta}$ is true in only a finite number of models of the class (4.17). So, for all tuples $(\alpha, \beta)$ satisfying (4.30) we have $\left[\Delta_{m}^{\alpha} \& \Delta_{n}^{\beta}\right]^{*}=0$ in the quotient modulo the Fréchet ideal. From this, we get $\left[\Delta_{m}\right]^{*}=\left[\Delta_{n}\right]^{*}$ by Lemma 0.1 (a), which shows that $\lambda(\nu(m))=\lambda(\nu(n))$.

We now check that $\lambda$ is an isomorphism.

(a) $\lambda$ preserves $\cap$ : Let $a$ and $b$ be arbitrary elements of $\mathcal{B}$, and $m, n, k$ be such that $a=\nu(m), b=\nu(n), k=v(m, n)$. Then we have $\nu(m) \cap \nu(n)=\nu(k)$. Let $p=\max \{m, n, k\}+1$. Then, for any sequence $\varepsilon=\left\langle\varepsilon_{0}, \varepsilon_{1}, \ldots\right\rangle$ for which

$$
\varepsilon \in 2^{<\omega},|\varepsilon| \geqslant p \text {, and }\left(\varepsilon_{m}, \varepsilon_{n}, \varepsilon_{k}\right) \in\{(1,1,0),(0,0,1),(0,1,1),(1,0,1)\} \text {, }
$$

we have $\varepsilon \notin$ Cons by (4.9). There are only finitely many sequences $\varepsilon$ of the form (4.31) with $|\varepsilon|=p$, while all other sequences of the class (4.31) are their extensions. Then by (4.15) we obtain that there are only finitely many sequences $\varepsilon \in \operatorname{Hlt}(\mathcal{M})$ for which condition (4.31) holds. Also, there can be only finitely many sequences $\varepsilon \in \operatorname{Hlt}(\mathcal{M})$ satisfying $|\varepsilon|<p$. Therefore, for any $\alpha, \beta, \gamma$ satisfying

$$
(\alpha, \beta, \gamma) \in\{(1,1,0),(0,0,1),(0,1,1),(1,0,1)\},
$$

the sentence $\Delta_{m}^{\alpha} \& \Delta_{n}^{\beta} \& \Delta_{k}^{\gamma}$ is true in only a finite number of models of the class (4.17). So, for all tuples $(\alpha, \beta, \gamma)$ satisfying (4.32) we have $\left[\Delta_{m}^{\alpha} \& \Delta_{n}^{\beta} \& \Delta_{k}^{\gamma}\right]^{*}=0$ in the quotient modulo the Fréchet ideal. From this, we get $\left[\Delta_{m}\right]^{*} \cap\left[\Delta_{n}\right]^{*}=$ $\left[\Delta_{k}\right]^{*}$ by Lemma $0.1(\mathrm{~b})$, which shows that $\lambda(\nu(m)) \cap \lambda(\nu(n))=\lambda(\nu(k))$.

Finally, we have

$$
\lambda(a \cap b)=\lambda(\nu(m) \cap \nu(n))=\lambda(\nu(k))=\lambda(\nu(m)) \cap \lambda(\nu(n))=\lambda(a) \cap \lambda(b),
$$

which means that $\lambda$ preserves the Boolean operation $\cap$.

(b) $\lambda$ preserves the operation - : Let $a$ be an arbitrary element of $\mathcal{B}$, and $m, n$ be such that $a=\nu(m), n=w(m)$. Then we have $-\nu(m)=\nu(n)$. Let $p=\max \{m, n\}+1$. Then, for any sequence $\varepsilon=\left\langle\varepsilon_{0}, \varepsilon_{1}, \ldots\right\rangle$, for which

$$
\varepsilon \in 2^{<\omega},|\varepsilon| \geqslant p \text {, and }\left(\varepsilon_{m}, \varepsilon_{n}\right) \in\{(0,0),(1,1)\},
$$

we have $\varepsilon \notin$ Cons by (4.9). There are only finitely many sequences $\varepsilon$ of the form (4.33) with $|\varepsilon|=p$, while all other sequences of the class (4.33) are their extensions. Then by (4.15) we obtain that there are only finitely many sequences $\varepsilon \in \operatorname{Hlt}(\mathcal{M})$ for which condition (4.33) holds. Also, there can be only finitely many sequences $\varepsilon \in \operatorname{Hlt}(\mathcal{M})$ satisfying $|\varepsilon|<p$. Therefore, for any $\alpha, \beta$ satisfying

$$
(\alpha, \beta) \in\{(0,0),(1,1)\},
$$


the sentence $\Delta_{m}^{\alpha} \& \Delta_{n}^{\beta}$ is true in only a finite number of models of the class (4.17). So, for all tuples $(\alpha, \beta)$ satisfying (4.34) we have $\left[\Delta_{m}^{\alpha} \& \Delta_{n}^{\beta}\right]^{*}=0$ in the quotient modulo the Fréchet ideal. From this, we get $-\left[\Delta_{m}\right]^{*}=\left[\Delta_{n}\right]^{*}$ by Lemma $0.1(\mathrm{c})$, which shows that $-\lambda(\nu(m))=\lambda(\nu(n))$.

Finally, we have

$$
\lambda(-a)=\lambda(-\nu(m))=\lambda(\nu(n))=-\lambda(\nu(m))=-\lambda(a),
$$

which means that $\lambda$ preserves the Boolean operation -.

(c) $\lambda$ preserves $\cup, 0$ and 1: We have by direct calculations: $\lambda(a \cup b)=$ $\lambda(-(-a \cap-b))=-(-\lambda(a) \cap-\lambda(b))=\lambda(a) \cup \lambda(b), \quad \lambda(0)=\lambda(0 \cap-0)=$ $\lambda(0) \cap-\lambda(0)=0$, and $\lambda(1)=\lambda(1 \cup-1)=\lambda(1) \cup-\lambda(1)=1$.

(d) $\lambda(a) \neq 0$ for $a \neq 0$. Let $a$ be an element of $\mathcal{B}$ such that $a \neq 0$. Choose $n$ such that $a=\nu(n)$. Since $\nu(n) \neq 0$, one can find a sequence $\varepsilon=\left\langle\varepsilon_{0}, \varepsilon_{1}, \ldots, \varepsilon_{n-1}\right\rangle \in 2^{<\omega}$ such that

$$
\nu(0)^{\varepsilon_{0}} \cap \nu(1)^{\varepsilon_{1}} \cap . . \cap \nu(n-1)^{\varepsilon_{n-1}} \cap \nu(n) \neq 0 .
$$

Thus, by definition (4.9), $\varepsilon \in$ Cons; moreover, $\varepsilon 1 \in$ Cons. Then, by properties (4.12) and (4.19), there exists an infinite sequence of zeros and ones $\varepsilon^{*}=\left\langle\varepsilon_{0}, \varepsilon_{1}, \varepsilon_{2}, \ldots\right\rangle \in$ Cons $^{*}$ whose initial subsequence of length $n+1$ is equal to $\varepsilon 1$. From this, it follows by (4.18) that the formula $\Delta_{n}$ is true in infinitely many finite models of $T$, for example, in all models of the form $\mathfrak{M}\left\langle\varepsilon_{0}, \varepsilon_{1}, \ldots, \varepsilon_{k}\right\rangle$, $k>n$. Thus $\left[\Delta_{n}\right]^{*} \neq 0$, establishing that $a \neq 0$ implies $\lambda(a) \neq 0$.

(e) $\lambda$ is "onto". This directly follows from Lemma 4.4 and the definition of $\lambda$.

From properties (a)-(e), it follows that the map

$$
\lambda: \mathcal{B} \rightarrow \mathcal{L}(\operatorname{ModFin}(T)) / \mathcal{F}
$$

is an isomorphism of Boolean algebras. The existence of general recursive functions $g_{1}(x)$ and $g_{2}(x)$ shown in Diagram (0.1) which represent the isomorphism $\lambda$ is obvious. Thus the mapping $\lambda$ is a constructive isomorphism between the numerated algebras $(\mathcal{B}, \nu)$ and $(\mathcal{L}(\operatorname{ModFin}(T)) / \mathcal{F}, \gamma / \mathcal{F})$.

So the constructive isomorphism (4.1) exists, and Theorem 1.5 is proven.

The scheme of the proof is completed.

Thus, to prove Theorem 1.5, we consider an arbitrary $\Sigma_{2}^{0}$ Boolean algebra $(\mathcal{B}, \nu)$ which satisfies the conditions $0 \neq 1$ and $(4.2)$, and for $(\mathcal{B}, \nu)$, we have to construct a Turing machine $\mathcal{M}$ satisfying the $P R G$ specification and to embed it in some finitely axiomatizable theory $T$ such that for the class of its finite models $\operatorname{ModFin}(T)$, the model-theoretic properties (4.17) and (4.25) are true.

We now make some more informal comments about the details of the construction. 
The reason for using extra zeros between the bit cells $\varepsilon_{i}$ is that they help prove statement (4.25). The special form of the set $\operatorname{Hlt}(\mathcal{M})$ forces the Lindenbaum algebra of the class of all finite models of the theory $T$ to satisfy (4.1). One can see that the set $\operatorname{Hlt}(\mathcal{M})$ formed by the machine is, in some sense, an approximation to the set Cons, defined by the numerated Boolean algebra $(\mathcal{B}, \nu)$. By (4.8), (4.13) and (4.14) both of them are trees and Cons $\subseteq \operatorname{Hlt}(\mathcal{M})$. Note that exact equality between these sets Cons $=\operatorname{Hlt}(\mathcal{M})$ is impossible in view of their different algorithmic complexity. However, the conditions (4.15) and (4.16) imply that the approximation of the set Cons by the set $\operatorname{Hlt}(\mathcal{M})$ is correct in some limiting sense, which is sufficient for proving the isomorphism (4.1), ignoring any finite joins of atoms in the Lindenbaum algebra considered.

The general description of the main goals of the construction is completed, and now we turn to the concrete details of the theory $T$.

\section{$5 \quad$ Programming technique}

A number cell with value $k$ is a sequence of $k+1$ ones on a tape which is bounded by blanks on both sides. A bit cell is a single tape cell which can be used in the program for the storage of Boolean information, represented by the symbols 0 or 1 .

Fig. 5.1 shows a flow chart of the algorithm, Fig. 5.2 and Fig 5.3 show all basic numeric and bit cells used during the computation of the Turing machine, and Fig. 5.4 represents the algorithm itself realized as a program of a Turing machine. Note that Fig. 5.3 has stages (a) $-(\mathrm{g})$, which are marked in both Figure 5.1 and Figure 5.4. They help us analyze the process of the algorithm, and also allow us to identify groups of instructions of the Turing machine which performs the operations in the flow chart.

The program consists of four main parts (which can be analyzed separately), namely: initial part, main cycle (e)-(b), local cycle with a subroutine (c)-(d), and final part. One can check that the program in Fig. 5.4 exactly corresponds to the flow-chart, and the extra information in Fig. 5.2 and Fig. 5.3 properly describes the algorithm.

We now make some more detailed comments.

To the left of the starting point, some complex Boolean information INPUT is located which consists of bit cells $\varepsilon_{0}, \varepsilon_{1}, \ldots$ and intervening sequences of zeros 


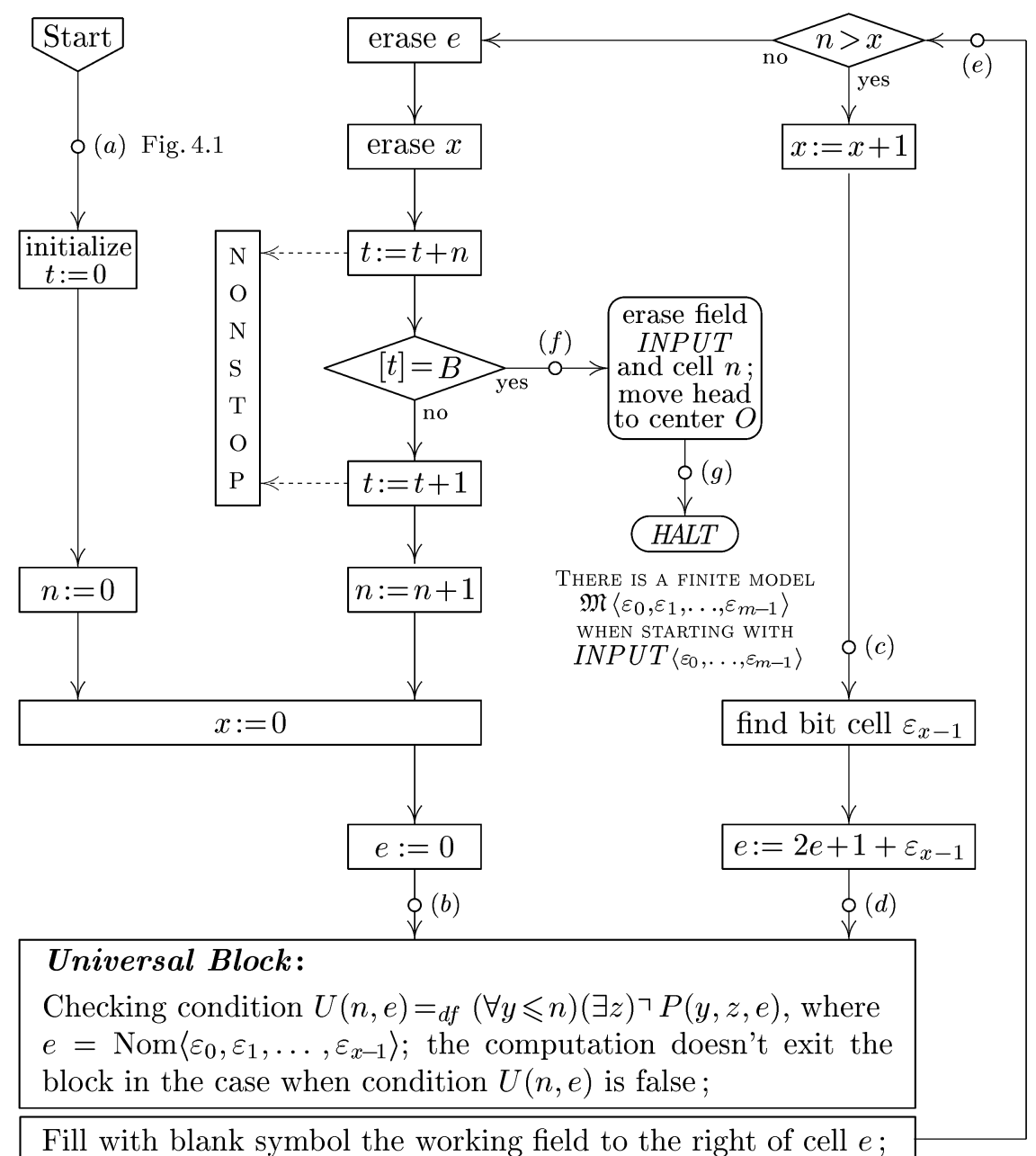

Fig. 5.1. Flow-chart of Turing machine $\mathcal{M}$

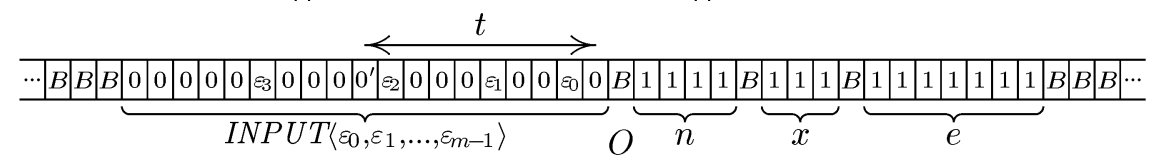

Fig. 5.2. Overall Scheme of fields and cells of a tape

\footnotetext{
$m$ - the number of bit cells $\varepsilon_{i}$ at INPUT (parameter weight of a model);

$t$ - current position of symbol $0^{\prime}$ in field INPUT (eg. $t=9$ in this figure);

$n$ - variable parameter for Main Cycle of computation (from 0 to $m$ );

$x$ - variable parameter for Local Cycle of computation (from 0 to $n$ );

$e$ - value of the tuple numeration function $\operatorname{Nom}\left\langle\varepsilon_{0}, \varepsilon_{1}, \ldots, \varepsilon_{x-1}\right\rangle$;
} 
(a)

q0 Start

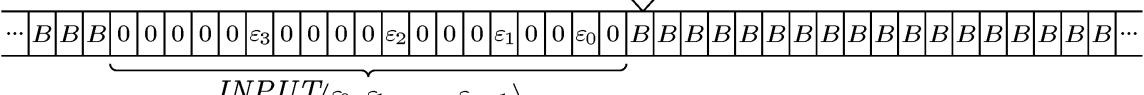
$\operatorname{INPUT}\left\langle\varepsilon_{0}, \varepsilon_{1}, \ldots, \varepsilon_{m-1}\right\rangle$

(b) An enter into the Universal Block $q_{75}$

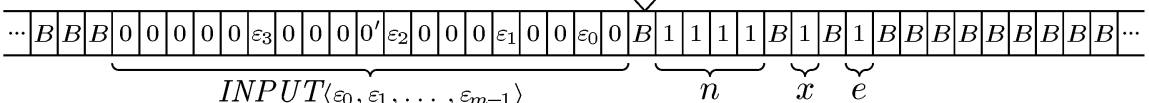

(c) An enter to compute by rule (2.2)

$q_{39}$

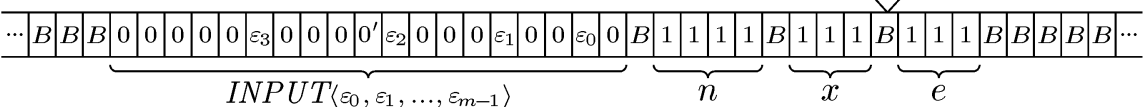

(d) An enter into the Universal Block $q_{75}$

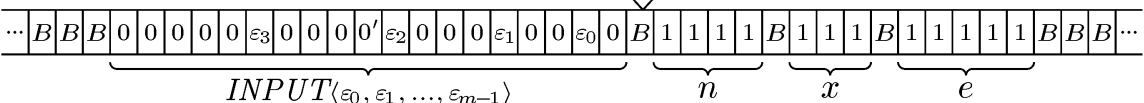

(e) The exit out of the Universal Block $q_{4}$

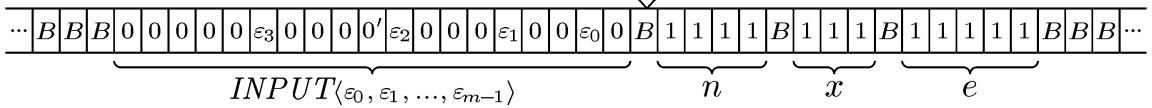

(f) $q_{23}$ End of the field INPUT detected

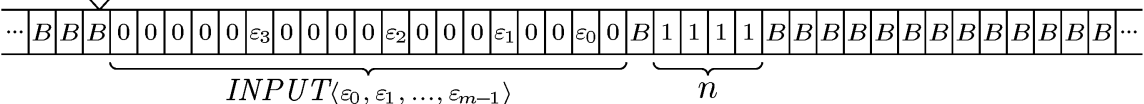

(g)

q74 Stop

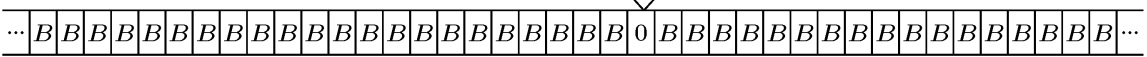

Fig. 5.3. Working states of Turing machine $\mathcal{M}$

of increasing length between them. There is a special numerical parameter $t$ acting in this field. The value of this parameter $t$ is represented by the position of a special symbol $0^{\prime}$ in the field INPUT. This symbol marks how far we have scanned the field INPUT up to the current stage of the algorithm. To the right side of the initial position of the head, we have the numerical cells $n, x$, and $e$. The cell $n$ is initialized at the beginning of the computation, and it remains active during the entire computation, while the cell $x$ is periodically erased, initialized and run over an increasing sequence of values. For each new value of $x$, the cell $e$ is computed to represent the value of $\operatorname{Nom}\left\langle\varepsilon_{0}, \varepsilon_{1}, \ldots, \varepsilon_{x-1}\right\rangle$.

Note that in the program there are four special commands controlling the information in the position of pointer $t$ in field INPUT. They are displayed in Fig. 5.4 by the use of arrows of the form $\mapsto$ (which are used only to show these special commands). These four commands ensure that the 


\begin{tabular}{|c|c|}
\hline $1 q_{14} \rightarrow B q_{14} L$ & $S T A R T$ \\
\hline$B q_{14} \rightarrow B q_{15} L$ & $(a)$ \\
\hline $1 q_{15} \rightarrow B q_{15} L$ & $B q_{0} \rightarrow B q_{1} L$ \\
\hline$B q_{15} \rightarrow B q_{16} L$ & $\begin{array}{l}0 q_{1} \rightarrow 0^{\prime} q_{2} R \\
B q_{2} \rightarrow B q_{3} R\end{array}$ \\
\hline $1 q_{16} \rightarrow 1 q_{17} L$ & $B q_{3} \rightarrow 1 q_{27} R$ \\
\hline $\begin{array}{l}1 q_{17} \rightarrow 1^{\prime} q_{18} L \\
B q_{17} \rightarrow B q_{22} L\end{array}$ & \\
\hline $0 q_{18} \rightarrow 0 q_{18} L$ & $(e)$ \\
\hline $1 q_{18} \rightarrow 1 q_{18} L$ & $B q_{4} \rightarrow B q_{5} R$ \\
\hline$B q_{18} \rightarrow B q_{18} L$ & $1 q_{5} \rightarrow 1^{\prime} q_{6} R$ \\
\hline $0^{\prime} q_{18} \rightarrow 0 q_{19} L$ & $1 q_{6} \rightarrow 1 q_{6} R$ \\
\hline $0 q_{19} \rightarrow 0^{\prime} q_{20} R$ & $B q_{6} \rightarrow B q_{7} R$ \\
\hline$B q_{19} \mapsto B q_{18} L$ & $1 q_{7} \rightarrow 1^{\prime} q_{8} L$ \\
\hline $1 q_{19} \mapsto 1 q_{18} L$ & \\
\hline $0 q_{20} \rightarrow 0 q_{20} R$ & $B q_{8} \rightarrow B q_{8} L$ \\
\hline $1 q_{20} \rightarrow 1 q_{20} R$ & $1 q_{8} \rightarrow 1 q_{8} L$ \\
\hline$B q_{20} \rightarrow B q_{20} R$ & $1^{\prime} q_{8} \rightarrow 1 q_{9} R$ \\
\hline $1^{\prime} q_{20} \rightarrow 1 q_{21} L$ & $1 q_{9} \rightarrow 1^{\prime} q_{10} R$ \\
\hline $1 q_{21} \rightarrow 1^{\prime} q_{18} L$ & $B q_{9} \rightarrow B q_{12} R$ \\
\hline$B q_{21} \rightarrow B q_{22} L$ & $\begin{array}{l}1 q_{10} \rightarrow 1 q_{10} R \\
B q_{10} \rightarrow B q_{10} R\end{array}$ \\
\hline $0 q_{22} \rightarrow 0 q_{22} L$ & $1^{\prime} q_{10} \rightarrow 1 q_{11} R$ \\
\hline $1 q_{22} \rightarrow 1 q_{22} L$ & $1 q_{11} \rightarrow 1^{\prime} q_{8} L$ \\
\hline $\begin{array}{l}0^{\prime} q_{22} \rightarrow 0 q_{23} L \\
0 q_{23} \rightarrow 0 q_{24} L\end{array}$ & $B q_{11} \rightarrow B q_{34} L$ \\
\hline $1 q_{23} \rightarrow 1 q_{24} L$ & $1 q_{12} \rightarrow 1 q_{12} R$ \\
\hline & $1^{\prime} q_{12} \rightarrow 1 q_{12} R$ \\
\hline$B q_{23} \rightarrow B q_{72} R$ & $B q_{12} \rightarrow B q_{13} R$ \\
\hline $0 q_{24} \rightarrow 0^{\prime} q_{25} R$ & $1 q_{13} \rightarrow 1 q_{13} R$ \\
\hline $1 q_{24} \mapsto 1 q_{23} R$ & $B q_{13} \rightarrow B q_{14} L$ \\
\hline $\begin{array}{l}B q_{24} \mapsto B q_{23} R \\
0 q_{25} \rightarrow 0 q_{25} R\end{array}$ & \\
\hline $1 q_{25} \rightarrow 1 q_{25} R$ & $1 q_{34} \rightarrow 1 q_{34} L$ \\
\hline$B q_{25} \rightarrow B q_{26} R$ & $B q_{34} \rightarrow B q_{34} L$ \\
\hline $1 q_{26} \rightarrow 1 q_{26} R$ & $1^{\prime} q_{34} \rightarrow 1 q_{35} R$ \\
\hline$B q_{26} \rightarrow 1 q_{27} R$ & $\begin{array}{l}1 q_{35} \rightarrow 1 q_{35} \Omega \\
B q_{35} \rightarrow B q_{36} R\end{array}$ \\
\hline$B q_{27} \rightarrow B q_{28} R$ & $1 q_{36} \rightarrow 1 q_{36} R$ \\
\hline$B q_{28} \rightarrow 1 q_{29} R$ & $B q_{36} \rightarrow 1 q_{37} R$ \\
\hline$B q_{29} \rightarrow B q_{30} R$ & $1 q_{37} \rightarrow B q_{38} R$ \\
\hline$B q_{30} \rightarrow 1 q_{31} L$ & $1 q_{38} \rightarrow 1 q_{38} R$ \\
\hline $\begin{array}{l}B q_{31} \rightarrow B q_{32} L \\
1 q_{32} \rightarrow 1 q_{33} L\end{array}$ & $B q_{38} \rightarrow 1 q_{39} L$ \\
\hline $\begin{array}{l}1 q_{32} \rightarrow 1 q_{33} L \\
B q_{33} \rightarrow B q_{75} L\end{array}$ & $1 q_{39} \rightarrow 1 q_{39} L$ \\
\hline $1 q_{75} \rightarrow 1 a_{75} L$ & \\
\hline
\end{tabular}

(b)
Final part of computation: $1 q_{72} \rightarrow B q_{72} R$ $0 q_{72} \rightarrow B q_{72} R$ $1 q_{73} \rightarrow B q_{73} R$ (c) $\begin{array}{ll}B q_{39} \rightarrow B q_{40} L & B q_{73} \rightarrow B q_{74} L \\ B q_{74} \rightarrow B q_{74} L\end{array}$ $1 q_{40} \rightarrow 1 q_{40} L \quad(g)$ $\begin{array}{ll}B q_{40} \rightarrow B q_{41} R & 0 q_{74} \rightarrow \text { STOP } \\ 1 q_{41} \rightarrow 1 q_{42} R & \end{array}$ $1 q_{42} \rightarrow 1^{\prime} q_{43} L$ $1 q_{43} \rightarrow 1^{\prime} q_{44} L$ $B q_{44} \rightarrow B q_{45} L$ $1 q_{45} \rightarrow 1 q_{45} L$ $B q_{45} \rightarrow B q_{46} L$ $0 q_{46} \rightarrow 0^{\prime} q_{47} R$

$0 q_{47} \rightarrow 0 q_{47} R$

$1 q_{47} \rightarrow 1 q_{47} R$

$B q_{47} \rightarrow B q_{47} R$

$1^{\prime} q_{47} \rightarrow 1 q_{48} R$

$1 q_{48} \rightarrow 1^{\prime} q_{55} L$

$1^{\prime} q_{48} \rightarrow 1^{\prime} q_{49} L$

$0 q_{49} \rightarrow 0 q_{49} L$

$1 q_{49} \rightarrow 1 q_{49} L$

$B q_{49} \rightarrow B q_{49} L$

$0^{\prime} q_{49} \rightarrow 0 q_{50} L$

$0 q_{50} \rightarrow 0^{\prime} q_{51} R$

$1 q_{50} \rightarrow 1^{\prime} q_{51} R$

$0 q_{51} \rightarrow 0 q_{51} R$

$1 q_{51} \rightarrow 1 q_{51} R$

$B q_{51} \rightarrow B q_{51} R$

$1^{\prime} q_{51} \rightarrow 1 q_{52} R$

$1 q_{52} \rightarrow 1^{\prime} q_{53} L$

$B q_{52} \rightarrow B q_{57} L$

$1 q_{53} \rightarrow 1 q_{53} L$

$B q_{53} \rightarrow B q_{54} R$

$1 q_{54} \rightarrow 1^{\prime} q_{55} L$

$0 q_{55} \rightarrow 0 q_{55} L$

$1 q_{55} \rightarrow 1 q_{55} L$

$B q_{55} \rightarrow B q_{55} L$

$0^{\prime} q_{55} \rightarrow 0 q_{56} L$

$1^{\prime} q_{55} \rightarrow 1 q_{56} L$

$0 q_{56} \rightarrow 0^{\prime} q_{47} R$
$0 q_{57} \rightarrow 0 q_{57} L$

$1 q_{57} \rightarrow 1 q_{57} L$

$B q_{57} \rightarrow B q_{57} L$

$0^{\prime} q_{57} \rightarrow 0 q_{58} R$

$1^{\prime} q_{57} \rightarrow 1 q_{63} R$

$0 q_{58} \rightarrow 0 q_{58} R$

$1 q_{58} \rightarrow 1 q_{58} R$

$B q_{58} \rightarrow B q_{59} R$

$1 q_{59} \rightarrow 1 q_{59} R$

$B q_{59} \rightarrow B q_{60} R$

$1 q_{60} \rightarrow 1 q_{60} R$

$B q_{60} \rightarrow B q_{61} R$

$1 q_{61} \rightarrow 1 q_{61} R$

$B q_{61} \rightarrow B q_{62} L$

$1 q_{62} \rightarrow 1^{\prime} q_{68} R$

$0 q_{63} \rightarrow 0 q_{63} R$

$1 q_{63} \rightarrow 1 q_{63} R$

$B q_{63} \rightarrow B q_{64} R$

$1 q_{64} \rightarrow 1 q_{64} R$

$B q_{64} \rightarrow B q_{65} R$

$1 q_{65} \rightarrow 1 q_{65} R$

$B q_{65} \rightarrow B q_{66} R$

$1 q_{66} \rightarrow 1 q_{66} R$

$B q_{66} \rightarrow 1 q_{67} L$

$1 q_{67} \rightarrow 1^{\prime} q_{68} R$

$1 q_{68} \rightarrow 1 q_{68} R$

$B q_{68} \rightarrow 1 q_{69} L$

$1 q_{69} \rightarrow 1 q_{69} L$

$1^{\prime} q_{69} \rightarrow 1 q_{70} L$

$1 q_{70} \rightarrow 1^{\prime} q_{68} R$

$B q_{70} \rightarrow B q_{71} L$ $1 q_{71} \rightarrow 1 q_{71} L$

$B q_{71} \rightarrow B q_{75} L$ $1 q_{75} \rightarrow 1 q_{75} L$ $(d)$

$B q_{75} \rightarrow \ldots$

Universal block: Checking condition $\left.U(n, e)=_{d f}(\forall y \leqslant n)(\exists z)\right\urcorner P(y, z, e)$ where $e=\operatorname{Nom}\left\langle\varepsilon_{0}, \ldots, \varepsilon_{x-1}\right\rangle$; If all quantifiers $\exists$ are successfully realized, computation is finished and exits the block in state $B q_{4}$ as shown in Fig. $5.3(\mathrm{e})$;

Fig. 5.4. The set of instructions of Turing machine $\mathcal{M}$ 
computation nonstops in the case when the left-hand side of the implication in (4.5) is true.

One can verify that every call of the subroutine (c)-(d) uses an initial segment of the field INPUT, which is located to the right of the current position of the pointer $t$, i.e., any computation of the subroutine uses a part of the bit information field which have already been checked in the computation.

During the computation, the symbol 0 in a tape cell can temporarily be replaced by the symbol $0^{\prime}$, and later, the previous symbol 0 will be restored in this cell. The same applies to the symbol $1^{\prime}$. Thus, the information in the field INPUT can be queried by the head of machine again and again; however, the information content of this field is not changed during the computation. As for cells $n, x$ and $e$, we perform both cell operations and cell-aided operations over them. A cell operation changes its value. A cell-aided operation does not change its value, but is performed to change some other cell, or to compare values of two cells.

The operations, represented in the flow chart, are organized in the form of back-and-forth movements of the machine head, using the symbols $0^{\prime}$ and $1^{\prime}$. In a sequence of back-and-forth movements of the head, the same routine is executed again and again while the amplitude of head movements is gradually increased (or decreased). For this reason, we call such computations quasiperiodic computations.

In addition to the quasi-periodic computations, there are entries into the so-called universal block, in which the following condition is checked for the current tape stage,

$$
U(n, e)=(\forall y \leqslant n)(\exists z)\urcorner P(y, z, e),
$$

where $n$ is the base cell of global cycle, while the current value of the cell $e$ represents the number of the sequence $\operatorname{Nom}\left\langle\varepsilon_{0}, \varepsilon_{1}, \ldots, \varepsilon_{x-1}\right\rangle$. So, condition (5.1) depends just on the parameters $n$ and $e$. The words "to check the condition $U(n, e)$ " mean that if the condition is false, the computation inside $U(n, e)$ never finishes its work (and so the computation as a whole does not stop). Otherwise, if the condition turns to be true, the computation finishes its work in a finite number of steps and then passes to further quasi-periodic computations according to the flow-chart.

The computations in the universal block can, of course, be programmed because the predicate $P(y, z, t)$ is recursive. But, for our construction, we have to use some particular form of the program of the universal block according to the flow-chart shown in Fig 5.5. It provides a quasi-monotonicity property of the computation that will be discussed in Section 10.

We now give some explanations of the details. 
One can see in Fig 5.5 that at the entry into the universal block, a subprogram called "Polynomial quasi-periodic subroutine" computes the value $c^{2}(n, e)$ (where $c(x, y)$ is the Cantor pairing function), and writes it in a special working cell $K E Y$ to the right of cell $e$. Then other parts of the universal block start to work, which we will discuss later. We call the subroutine of the computation $c^{2}(n, e)$ the polynomial part of the computation in the universal block. It is assumed that this subroutine is programmed as (quite complex) quasi-periodic computation, whose properties were discussed earlier.

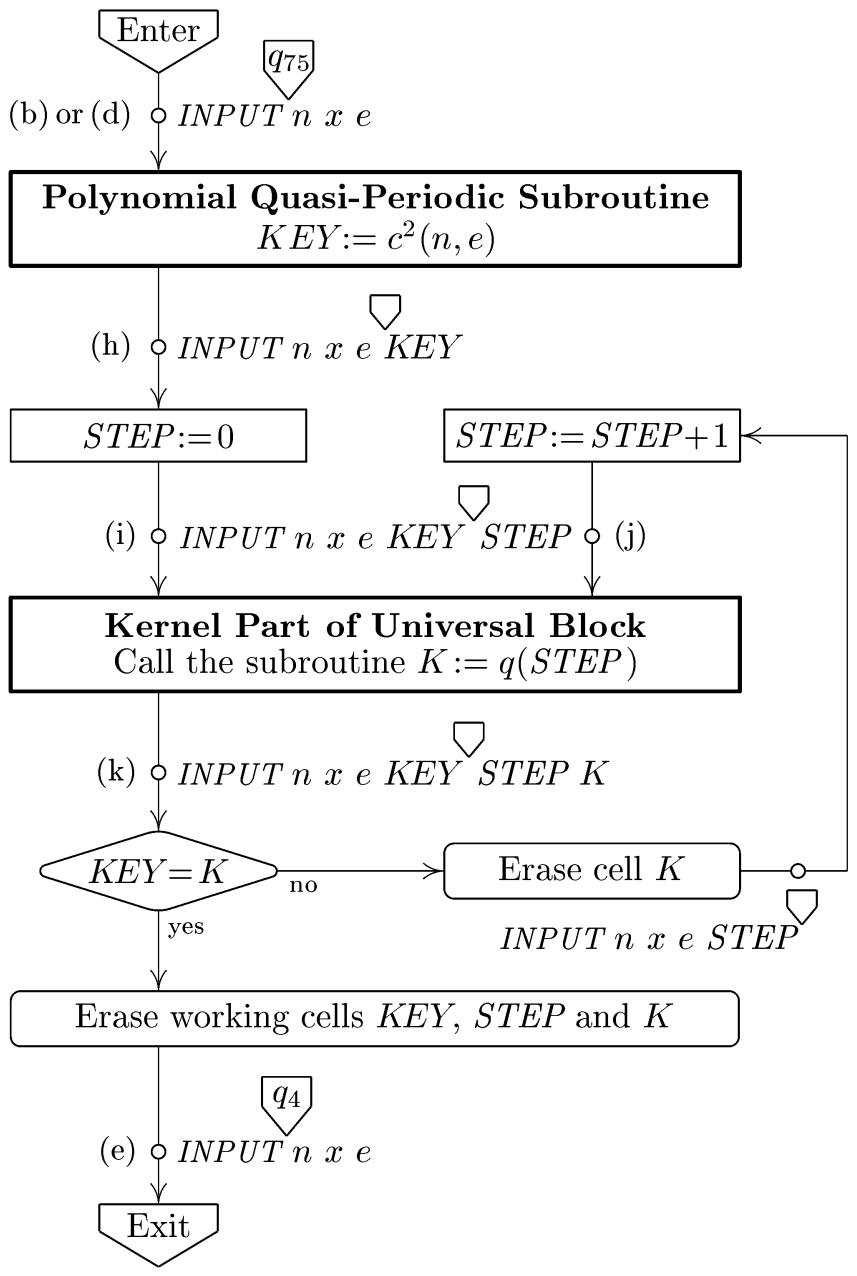

Fig. 5.5. Internal Structure of the Universal Block 
Consider the following new relation

$$
\begin{aligned}
U^{\prime}(n, e) \Leftrightarrow & U(n, e) \text { is true and a call } U(n, e) \text { can occur in } \\
& \text { a computation in a model of the class (4.17). }
\end{aligned}
$$

Obviously, $U^{\prime}(n, e) \Rightarrow U(n, e)$ for all $n, e \in \mathbb{N}$. Moreover, we have the following direct representation for the relation $U^{\prime}(n, e)$ :

$$
\begin{aligned}
& U^{\prime}(n, e) \Leftrightarrow \\
& e=\operatorname{Nom}\left\langle\varepsilon_{0}, \ldots, \varepsilon_{x-1}\right\rangle \&(x \leqslant n) \&(\forall j \leqslant x) U\left(n, \operatorname{Nom}\left\langle\varepsilon_{0}, \ldots, \varepsilon_{j-1}\right\rangle\right) .
\end{aligned}
$$

Note two technical facts.

Lemma 5.1. For all $n, n_{1}, e \in \mathbb{N}$, if $n>n_{1}$ and $U^{\prime}(n, e)$ is true, then $U^{\prime}\left(n_{1}, e\right)$ is true.

Proof. Immediately from (5.1) and (5.2).

Lemma 5.2. Let $\varepsilon \in$ Cons be given, and $e=\operatorname{Nom}\langle\varepsilon\rangle$. Then $U^{\prime}(n, e)$ is true for all $n \in \mathbb{N}$.

Proof. Directly from (4.10), (5.1) and (5.2).

Consider the following auxiliary sequence of integers:

$$
V=\{k \cdot(k+1) \mid k>0\} .
$$

Note that no elements in $V$ are pure squares because $n^{2}<n \cdot(n+1)<(n+1)^{2}$ for all $n>0$.

By definitions (5.1) and (5.2), the set

$$
W=\left\{(n, e) \mid U^{\prime}(n, e)\right\}
$$

is recursively enumerable. By (4.11) and (4.14), we have $(0,0) \in W$. Furthermore, by (4.11) we have \langle\rangle$\in$ Cons, and therefore by Lemma 5.2 , we have $(n, 0)$ for all $n \in \mathbb{N}$. In particular, the set $W$ is infinite. Let $w_{0}(x)$ and $w_{1}(x)$ be two general recursive functions such that $w_{0}(0)=w_{1}(0)=0$ and

$$
W=\left\{\left(w_{0}(j), w_{1}(j)\right) \mid j \in \mathbb{N}\right\}
$$

is an effective enumeration of the set $W$ without repetitions.

Consider a general recursive function $q(x)$ such that

$$
\{q(j) \mid j \in \mathbb{N}\}=\left\{c^{2}(n, e) \mid(n, e) \in W\right\} \cup V^{\prime}
$$

is an enumeration of the set $c^{2}(W) \cup V^{\prime}$ for some subset $V^{\prime} \subseteq V$ (possibly with repetitions), where the function $q(x)$ is constructed by the method described 
below and is such that the following properties hold:

$$
\begin{aligned}
& (\forall x)[q(x) \leqslant x], \\
& (\forall y)(\exists z)(\forall x \geqslant z)[q(x) \geqslant y] .
\end{aligned}
$$

The function $q(x)$ is defined in steps $s=0,1,2, \ldots$ as follows.

Step $s=0$. We set $q(0)=0$.

Assume step $s$ of the construction is completed and $q(x)$ is defined on the interval $\left[0, k_{s}\right]$.

Step $s+1$. Let $(n, e)$ be the $(s+1)$-th pair in the enumeration (5.5) of the set $W$, i.e., $(n, e)=\left(w_{0}(s+1), w_{1}(s+1)\right)$. Let $e=\operatorname{Nom}\left\langle\varepsilon_{0}, \varepsilon_{1}, \ldots, \varepsilon_{x-1}\right\rangle$ with $x \geq 1$. Obviously, by (5.2), we have $n>0$. Consider the following sequence of numbers:

$$
\begin{aligned}
& e_{0}=\operatorname{Nom}\langle\rangle, \\
& e_{1}=\operatorname{Nom}\left\langle\varepsilon_{0}\right\rangle, \\
& e_{2}=\operatorname{Nom}\left\langle\varepsilon_{0}, \varepsilon_{1}\right\rangle, \\
& \ldots \\
& e_{x-1}=\operatorname{Nom}\left\langle\varepsilon_{0}, \varepsilon_{1}, \ldots, \varepsilon_{x-1}\right\rangle .
\end{aligned}
$$

We want to define the values of $q(x)$ for $x>k_{s}$ so as to cover all the elements of the following sequence in the order that they appear.

$$
\begin{aligned}
& c^{-}\left(n, e_{0}\right), c^{+}\left(n, e_{0}\right), c^{2}\left(n, e_{0}\right), c^{-}\left(n, e_{1}\right), c^{+}\left(n, e_{1}\right), c^{2}\left(n, e_{1}\right), \ldots \\
& \ldots, c^{-}\left(n, e_{x-1}\right), c^{+}\left(n, e_{x-1}\right), c^{2}\left(n, e_{x-1}\right) .
\end{aligned}
$$

Here, we use functions

$$
\begin{aligned}
& c^{-}(n, e)=[c(n, e)-1] \cdot c(n, e), \\
& c^{+}(n, e)=c(n, e) \cdot[c(n, e)+1], \\
& c^{2}(n, e)=c(n, e) \cdot c(n, e) .
\end{aligned}
$$

Because of the restriction (5.7), we may not be able to insert each element from this sequence immediately as a value of $q(x)$. Therefore, suppose that $q(x)$ is already defined for all $x<k$, and we are trying to define $q(k)$ to include the next value $c$ from the sequence (5.9) (for example, $c=c^{2}\left(n, e_{1}\right)$ ). If $c \leqslant k$, we simply set

$$
q(k)=c .
$$

Otherwise, if $c>k$, we set

$$
\begin{aligned}
& (\forall x \in[k, c-1])[q(x)=(\max b \leqslant x)(\exists y<x)[q(y)=b]], \\
& \text { and } \\
& q(c)=c .
\end{aligned}
$$


This completes the construction of $q(x)$.

Notice that we set $q(k)=c$ if $c$ is not too large. Otherwise, if $c>k$, we add some additional values of $q(x)$ for $x$ in interval $[k, c-1]$ to avoid contradicting (5.7), and finally we set $q(c)=c$. As a result, we have added $c$ to the values of the function $q$ such that (5.7) remains satisfied. At the same time, the entire construction of $q(x)$ will satisfy condition (5.8) because the enumeration (5.5) does not have any repetitions, and we choose as additional values of $q(x)$ in the interval $[k, c-1]$ of $(5.10)$ elements $b$ as large as possible which occurred earlier. So, conditions (5.7) and (5.8) are both satisfied by the construction of $q$.

Note that the elements $c^{2}\left(n, e_{0}\right), c^{2}\left(n, e_{1}\right), \ldots, c^{2}\left(n, e_{x-1}\right)$ of (5.9) must belong to the set $c^{2}(W)$ by Lemma 5.1 since we have $(n, e)=\left(n, e_{x-1}\right) \in W$. All other elements in (5.9) are included in the set $V$ since we have $2 \leqslant c\left(n, e_{0}\right)<$ $c\left(n, e_{1}\right)<\ldots$ in our case with $n>0$.

The idea of the program $U(n, x)$ shown in Fig. 5.5 is quite obvious. After the cell $K E Y$ is computed as discussed above, the program initializes and then runs a cycle over a numeric cell $S T E P$ for $S T E P=0,1,2, \ldots$. At each stage $S T E P$ of the cycle, we call a special subroutine to compute $q(x)$ at the argument $x=S T E P$, and the result is written into a special cell $K$. This part of the universal block is called the kernel part of the universal block. Since $q(x)$ is a general recursive function, any call of the kernel part finishes its work in a finite number of steps and yields some result. Then a special subroutine starts, which checks if

$$
K E Y=K
$$

holds. If (5.11) is true, we exit the universal block (previously clearing all used working fields), otherwise, the same computation is executed for the next value of the cycle cell STEP, etc.

The following fact holds:

Lemma 5.3. Let the universal block be programmed as in the flow-chart shown in Fig. 5.5. If $U(n, e)$ is called during the computation of the machine $\mathcal{M}$ with parameters $n$ and $e$ in a model of the class (4.17), then it finishes its work if and only if condition (5.1) is true for given values of $n$ and $e$.

Proof. First suppose that $U(n, e)$ is true. Then this pair $(n, e)$ is included in the set (5.4)-(5.5) and therefore $c^{2}(n, e)$ is included in the set (5.6). Thus, for an appropriate value of cell STEP, equation (5.11) is true, which causes the exit out of the universal block. Now, suppose that $U(n, e)$ is false and therefore $U^{\prime}(n, e)$ is false as well. This means that the pair $(n, e)$ does not belong to the set (5.5), and therefore, the number $c^{2}(n, e)$ cannot belong to the set (5.6) by property (2.5). So, the computation by the scheme given in Fig. 5.5 never finishes its work because the truth of (5.11) fails at every instance of the STEPcycle. 
Thus, the routine we have in Fig 5.5 satisfies condition (5.1).

Lemma 5.4. For any $n$ and $e$, the computation $U(n, e)$ loops through its internal STEP cycle at least $n$ times.

Proof. Immediate from the flow-chart shown in Fig 5.5, using the inequalities (2.6) and (5.7).

The computations in the universal block can be programmed because the function $q(x)$ is recursive. For our construction, it is assumed that all the cell and cell-aided operations used in Fig. 5.5 over cells $n, x, e, K E Y, S T E P$ and $K$ are programmed as quasi-periodic computations. This part of the program of the universal block looks simple enough, and therefore its details (i.e., Turing machine instructions) are omitted here. As for the subprogram in the kernel of the universal block, its particular form is irrelevant for our construction. It is important for us only that this part can be programmed.

The description of the details of the universal block is finished.

There are three general requirements on the program for the universal block:

a computation in the universal block should act on the right side

of the tape, and it should not pass its head farther than

the left bound of the cell $n$;

the kernel computation in the universal block should not pass its

head farther than the left bound of the cell STEP;

there should be a subroutine at the exit of the universal block which

clears the working field and restores the values of $n, x$ and $e$ that they

had at the entry of the block (see the form of exit stage in Fig. $5.3(e)$ ).

So the universal block must work on the right side of the tape; moreover, all input parameters needed for its work are presented with current cells $n, x$ and $e$, which are accessible to the block by (5.12). When the computation $U(n, x)$ is finished, the program should clear its working field just before the exit of the block.

Now, we consider what the program computes as a whole when the input information is correct, i.e., it has the form of $\operatorname{INPUT}\left\langle\varepsilon_{0}, \ldots, \varepsilon_{m-1}\right\rangle$ for a sequence $\left\langle\varepsilon_{0}, \ldots, \varepsilon_{m-1}\right\rangle \in \operatorname{Hlt}(\mathcal{M})$.

According to the flow chart shown in Fig 5.1, at its start, the machine initializes the position of the pointer $t$ and the value of the cell $n$ and then some cycle with base cell $n$ is started which we call a global cycle. In each instance of this global cycle (except for the 0th instance), the value of $n$ is increased by 1 while $n \leqslant$ weight $(I N P U T)$. For each new value of $n$, we arrange for a special local cycle which uses the cell $x$ with values from 0 to $n$ and which makes entries into 
the universal block. The cell $e$ is initialized when the value of $x$ is initialized, and for each new value of $x$, we set $e$ to be the Gödel number of the tuple $\left\langle\varepsilon_{0}, \varepsilon_{1}, \ldots, \varepsilon_{x-1}\right\rangle$ as calculated by the inductive rule (2.2). As a result of the execution of the cycle with $n$ and the cycles with $x$, we will have checked the sequence of conditions

$$
\begin{aligned}
& U(n, e), \quad 0 \leqslant n \leqslant m, 0 \leqslant x \leqslant n, e=\operatorname{Nom}\left\langle\varepsilon_{0}, \ldots, \varepsilon_{x-1}\right\rangle, \\
& \text { where } m=\operatorname{weight}(I N P U T) .
\end{aligned}
$$

When the pointer $t$ reaches a blank symbol (where a bit cell $\varepsilon_{i}$ should be), the main cycle is finished, and the final part of computation is performed. This part erases the field INPUT and the cell $n$, and finally stops in the state shown in Fig. 5.3 (g). So one can see that if at least one of the conditions (5.15) turns out to be false, the computation can never reach a halt state; otherwise, if all of them are true, the computation must reach a halt state.

Now, we prove a few lemmas concerning the algorithm.

Lemma 5.5. Let the machine $\mathcal{M}$ start with a tape situation $\mathcal{S} \in$ SpecST different from the form INPUT $\left\langle\varepsilon_{0}, \varepsilon_{1}, \ldots, \varepsilon_{m-1}\right\rangle$ with finite $m$. Then $\mathfrak{M}$ will not reach a halt state in this computation.

Proof. The machine $\mathcal{M}$ cannot reach a halting state in such an $\mathcal{S}$-computation because it checks the input information. If the input turns out to be wrong in a cell inside a separating field of 0's, a special command marked with $\mapsto$ will apply, resulting in an infinite cycle.

Lemma 5.6. Let the machine $\mathcal{M}$ start with $\mathcal{S}=\operatorname{INPUT}\left\langle\varepsilon_{0}, \ldots, \varepsilon_{m-1}\right\rangle$. Then $\mathcal{M}$ reaches a halt state in the $\mathcal{S}$-computation if and only if the following condition holds: $(\forall n \leqslant m)(\forall x \leqslant n)(\forall y \leqslant n)(\exists z)\urcorner P\left(y, z, \operatorname{Nom}\left\langle\varepsilon_{0}, \ldots, \varepsilon_{x-1}\right\rangle\right)$.

Proof. Since the input information is correct in this case, the special commands marked with $\mapsto$ never apply. Therefore, the $\mathcal{S}$-computation will reach a halt state if and only if all of the conditions (5.15) are true. This is exactly equivalent to the truth of the expression in Lemma 5.6.

Lemma 5.7 The machine $\mathcal{M}$ meets all requirements of the specification $P R G$.

Proof. One can see that the flow-chart of the machine $\mathcal{M}$ given in Fig. 5.1 makes an adequate representation of the specification $P R G$. Moreover, requirements (4.5) and (4.6) are guaranteed by Lemma 5.5 and Lemma 5.6.

So, with the Turing machine $\mathcal{M}$ constructed above, all properties (4.5)(4.16), (4.18)-(4.23), including Lemma 4.1, are true because they are all deduced in Section 4 from the statements (4.2)-(4.6). As for (4.17), this property will be discussed later after the theory $T$ is completely described.

The gist of the program $\mathcal{M}$ consists of properly arranged quasi-periodic 
computations (with very simple tape configurations) preparing cells $n, x, e$, $K E Y, S T E P$, and $K$ as needed, and entries to the kernel subroutine of the universal block. As a result, it will be possible to completely classify all types of local areas in models of the theory $T$. This will ensure the necessary modeltheoretic properties of the resulting theory.

A more detailed analysis of the operation of the algorithm and its effect on the properties of the theory will be considered later, after the exact formal representation of the theory $T$ has been given in the following section.

\section{Axiomatic and base properties of models}

Let $\mathcal{M}$ be the Turing machine constructed in the previous section, and let $d$ and $e$ be the corresponding parameters denoting the number of tape symbols and the number of states (here, $d=5$ ). We describe a finitely axiomatizable theory $T=F(\mathcal{B}, \nu)$, which, as will be shown later, has all the properties required by Theorem 1.5. The signature of the theory $T$ is

$$
\sigma_{0}=\left\{D^{2}, H^{2}, S^{2}, N^{2}, O, J, B, B L, B R, C T, C L, C R, A_{0}, \ldots, A_{d-1}, Q, Q_{0}, \ldots, Q_{e-1}\right\} .
$$

Here, the superscripts denote the arities of the predicate symbols and the symbols without superscripts are unary predicate symbols. Note that $\sigma_{0}$ includes all the predicates in (3.3).

The axioms of $T$ are the following sentences, grouped according to their meaning in the theory.

Axioms of the frame base: $H$ is an equivalence relation giving the horizontal rows, $D$ links each row to the row above:

$$
\begin{aligned}
& 1^{\circ} .(\forall x) H(x, x) \\
& 2^{\circ} .(\forall x)(\forall y)[H(x, y) \rightarrow H(y, x)] \\
& 3^{\circ} .(\forall x)(\forall y)(\forall z)[H(x, y) \& H(y, z) \rightarrow H(x, z)] \\
& 4^{\circ} .(\forall x)(\forall y)(\forall u)(\forall v)[H(x, u) \& H(y, v) \rightarrow(D(x, y) \leftrightarrow D(u, v))] \\
& 5^{\circ} .(\forall x)(\forall y)(\forall u)(\forall v)[D(x, y) \& D(u, v) \rightarrow(H(x, u) \leftrightarrow H(y, v))] \\
& \left.6^{\circ} .(\forall x)[\urcorner D(x, x)\right]
\end{aligned}
$$

Axioms of endpoints: $C T$ gives the top corner of the triangle, and $J$ the bottom row:

$$
\begin{aligned}
& \left.7^{\circ} .(\forall x)[C T(x) \leftrightarrow\urcorner(\exists y) D(x, y)\right] \\
& 8^{\circ} .(\forall x)(\forall y)[C T(x) \& C T(y) \rightarrow(x=y)] \\
& \left.9^{\circ} .(\forall x)[J(x) \leftrightarrow\urcorner(\exists y) D(y, x)\right]
\end{aligned}
$$


10․ $(\forall x)(\forall y)[J(x) \& J(y) \rightarrow H(x, y)]$

Axioms of the central point: $O$ gives the center of the bottom row:

$11^{\circ} .(\exists x)[J(x) \& O(x)]$

$12^{\circ} .(\forall x)(\forall y)[O(x) \& O(y) \rightarrow(x=y)]$

Axioms of the skeleton: $S$ and $N$ link a point to its neighbor to the right or above, respectively:

$$
\begin{aligned}
& \left.13^{\circ} .(\forall x)[\urcorner S(x, x)\right] \\
& 14^{\circ} .(\forall x)(\forall y)(\forall u)(\forall v)[S(x, y) \& S(u, v) \rightarrow((x=u) \leftrightarrow(y=v))] \\
& 15^{\circ} .(\forall x)(\forall y)(\forall u)(\forall v)[N(x, y) \& N(u, v) \rightarrow((x=u) \leftrightarrow(y=v))] \\
& 16^{\circ} .(\forall x)(\forall y)(\forall u)(\forall v)[N(x, y) \& N(u, v) \rightarrow(S(x, u) \leftrightarrow S(y, v))] \\
& 17^{\circ} .(\forall x)(\forall y)[S(x, y) \rightarrow H(x, y)] \\
& 18^{\circ} .(\forall x)(\forall y)[N(x, y) \rightarrow D(x, y)]
\end{aligned}
$$

Axioms of the boundaries: $B$ gives both upper boundaries of the triangle, with $B L$ and $B R$ forming the left and right diagonal boundaries of the triangle:

$$
\begin{aligned}
& \left.19^{\circ} .(\forall x)[B(x) \leftrightarrow\urcorner(\exists y) N(x, y)\right] \\
& 20^{\circ} .(\forall x)[B(x) \leftrightarrow B L(x) \vee B R(x)] \\
& \left.21^{\circ} .(\forall x)[B L(x) \leftrightarrow\urcorner(\exists y) S(y, x)\right] \\
& \left.22^{\circ} .(\forall x)[B R(x) \leftrightarrow\urcorner(\exists y) S(x, y)\right] \\
& 23^{\circ} .(\forall x)(\forall y)(\forall z)[S(x, y) \& N(y, z) \rightarrow(B L(x) \leftrightarrow B L(z))] \\
& 24^{\circ} .(\forall x)(\forall y)(\forall z)[S(x, y) \& N(x, z) \rightarrow(B R(y) \leftrightarrow B R(z))] \\
& \left.25^{\circ} .(\forall x)(\forall y)[B L(x) \& B R(y) \rightarrow\urcorner S(x, y)\right] \\
& 26^{\circ} .(\forall x)(\exists y)[H(x, y) \& B L(y)] \\
& 27^{\circ} .(\forall x)(\exists y)[H(x, y) \& B R(y)] \\
& 28^{\circ} .(\forall x)(\forall y)[H(x, y) \& B L(x) \& B L(y) \rightarrow(x=y)] \\
& 29^{\circ} .(\forall x)(\forall y)[H(x, y) \& B R(x) \& B R(y) \rightarrow(x=y)] \\
& \left.30^{\circ} .(\forall x)[J(x) \leftrightarrow\urcorner(\exists y) N(y, x)\right]
\end{aligned}
$$

Axioms of the corners and boundaries: We now relate corners and boundaries:

$$
\begin{aligned}
& 31^{\circ} .(\forall x)[C T(x) \leftrightarrow B L(x) \& B R(x)] \\
& 32^{\circ} .(\forall x)[C L(x) \leftrightarrow B L(x) \& J(x)] \\
& 33^{\circ} .(\forall x)[C R(x) \leftrightarrow B R(x) \& J(x)]
\end{aligned}
$$

Axioms of the possible forms of information: $A_{i}$ and $Q_{j}$ denote that 
a point bears symbol $a_{i}$ or is in state $q_{j}$, respectively; and $Q$ denotes that a point is scanned by the head (i.e., is in at least one state):

$$
\begin{aligned}
& 34^{\circ} .(\forall x)\left[A_{0}(x) \vee A_{1}(x) \vee \ldots \vee A_{d-1}(x)\right] \\
& \left.\left.35^{\circ} .(\forall x)[\urcorner A_{i}(x) \vee\right\urcorner A_{j}(x)\right], 0 \leqslant i<j<d \\
& 36^{\circ} .(\forall x)\left[Q(x) \leftrightarrow Q_{0}(x) \vee Q_{1}(x) \vee \ldots \vee Q_{e-1}(x)\right] \\
& \left.\left.37^{\circ} .(\forall x)[\urcorner Q_{i}(x) \vee\right\urcorner Q_{j}(x)\right], 0 \leqslant i<j<e
\end{aligned}
$$

Axioms of the starting position: We start in the bottom row in state $q_{0}$ and place restrictions on the symbols $a_{i}$ that can appear in the bottom row:

$$
\begin{aligned}
& 38^{\circ} .(\forall x)\left[O(x) \rightarrow Q_{0}(x)\right] \\
& 39^{\circ} .(\forall x)\left[J(x) \rightarrow A_{0}(x) \vee A_{1}(x) \vee A_{2}(x)\right] \\
& 40^{\circ} .(\forall x)(\forall y)\left[S(x, y) \& O(y) \rightarrow A_{0}(x) \& A_{2}(y)\right] \\
& \left.41^{\circ} .(\forall x)(\forall y)\left[S(x, y) \& J(y) \& A_{2}(y) \&\right\urcorner O(y) \rightarrow A_{2}(x)\right] \\
& 42^{\circ} .(\forall x)\left[C L(x) \rightarrow A_{2}(x)\right] \\
& 43^{\circ} .(\forall x)\left[C R(x) \rightarrow A_{2}(x)\right]
\end{aligned}
$$

Head location axiom: Each row contains a unique point being scanned by the head, which is not on a diagonal boundary:

$$
\begin{aligned}
& 44^{\circ} .(\forall x)(\forall y)[H(x, y) \& Q(x) \& Q(y) \rightarrow(x=y)] \\
& \left.45^{\circ} .(\forall x)[Q(x) \rightarrow\urcorner B(x)\right]
\end{aligned}
$$

Axiom of preserving a tape symbol: Only a point that is scanned can bear a different symbol in the row above:

$$
\left.46^{\circ} .(\forall x)(\forall y)[\urcorner Q(x) \& A_{i}(x) \& N(x, y) \rightarrow A_{i}(y)\right], 0 \leqslant i<d
$$

Axioms of the Turing machine instructions: Each non-bottom row is determined from the row below by a Turing machine instruction:

$47^{\circ} .(\forall u)\left[A_{i}(u) \& Q_{j}(u) \rightarrow(\exists x)(\exists y) N(u, y) \& S(x, y) \& A_{s}(y) \& Q_{t}(x)\right]$, for all commands $\langle i, j, s, t, L\rangle \in \mathcal{M}$

$48^{\circ} .(\forall u)\left[A_{i}(u) \& Q_{j}(u) \rightarrow(\exists x)(\exists y) N(u, x) \& S(x, y) \& A_{s}(x) \& Q_{t}(y)\right]$, for all commands $\langle i, j, s, t, R\rangle \in \mathcal{M}$

Axiom of the halting configuration: We halt when no Turing machine instruction applies:

$49^{\circ} .(\forall x)\left[A_{i}(x) \& Q_{j}(x) \rightarrow(\exists y)(N(x, y) \& C T(y))\right]$, for all $i, j$ such that $\langle i, j$, stop $\rangle \in \mathcal{M}$

\section{Corollaries}

$$
\mathrm{C} 50^{\circ} .(\forall x)(\forall y)(\forall u)(\forall v)[S(x, y) \& S(u, v) \rightarrow(N(x, u) \leftrightarrow N(y, v))]
$$




$$
\begin{aligned}
& \left.\left.\mathrm{C} 51^{\circ} .(\forall x)(\forall y)[S(x, y) \rightarrow\urcorner N(x, y) \&\right\urcorner N(y, x)\right] \\
& \left.\mathrm{C} 52^{\circ} .(\forall x)[\urcorner N(x, x)\right] \\
& \mathrm{C} 53^{\circ} .(\forall x)[(\exists y) N(x, y) \leftrightarrow(\exists z) S(z, x)] \\
& \mathrm{C} 54^{\circ} .(\forall x)[(\exists y) N(x, y) \leftrightarrow(\exists z) S(x, z)] \\
& \left.\mathrm{C} 55^{\circ} .(\forall x)[C T(x) \rightarrow\urcorner Q(x)\right] \\
& \mathrm{C} 56^{\circ} .(\forall x)(\forall y)[C T(x) \& N(y, x) \rightarrow Q(y)] \\
& \mathrm{C} 57^{\circ} .(\forall u)(\forall x y z)[Q(u) \& N(y, u) \& S(x, y) \& S(y, z) \rightarrow Q(x) \vee Q(z)] \\
& \left.\mathrm{C} 58^{\circ} .(\forall x)[B(x) \&\urcorner C T(x) \rightarrow A_{2}(x)\right] \\
& \mathrm{C} 59^{\circ} .(\exists x) C T(x) \\
& \mathrm{C} 60^{\circ} .(\exists x) C L(x) \\
& \mathrm{C} 61^{\circ} .(\exists x) C R(x) \\
& \mathrm{C} 62^{\circ} .(\forall x)(\forall y)[C L(x) \& C L(y) \rightarrow(x=y)] \\
& \mathrm{C} 63^{\circ} .(\forall x)(\forall y)[C R(x) \& C R(y) \rightarrow(x=y)] \\
& \left.\left.\mathrm{C} 64^{\circ} .(\forall x)[\urcorner C T(x) \vee\right\urcorner C L(x)\right] \\
& \left.\left.\mathrm{C} 65^{\circ} .(\forall x)[\urcorner C T(x) \vee\right\urcorner C R(x)\right] \\
& \left.\left.\mathrm{C} 66^{\circ} .(\forall x)[\urcorner C L(x) \vee\right\urcorner C R(x)\right] \\
& \mathrm{C} 67^{\circ} .(\forall x)(\forall y)[H(x, y) \rightarrow(J(x) \leftrightarrow J(y))] \\
& \mathrm{C} 68^{\circ} .(\forall x)(\forall y)[S(x, y) \rightarrow(J(x) \leftrightarrow J(y))] \\
& \mathrm{C} 69^{\circ} .(\forall x)[O(x) \leftrightarrow J(x) \& Q(x)] \\
& \mathrm{C} 70^{\circ} .(\forall x)[O(x) \rightarrow \neg B(x)]
\end{aligned}
$$

It is possible to consider the corollaries as additional axioms which help to better explain the theory. However, all of them can be deduced (though not always obviously) from the axioms, taking into account that we only consider finite models.

As defined above, let $\operatorname{INPUT}\left\langle\varepsilon_{0}, \varepsilon_{1}, \ldots, \varepsilon_{m-1}\right\rangle, \varepsilon_{i} \in\{0,1\}$, denote the initial tape configuration of $\mathcal{M}$ as in Fig. 4.1, where the bit cells $\varepsilon_{i}$ on the tape are from the sequence $\left\langle\varepsilon_{0}, \varepsilon_{1}, \ldots, \varepsilon_{m-1}\right\rangle$. We say that a finite model $\mathfrak{M}$ of the theory $T$ represents a starting state of type $\operatorname{INPUT}\left\langle\varepsilon_{0}, \varepsilon_{1}, \ldots, \varepsilon_{m-1}\right\rangle$ if its $J$-class represents some interval from the infinite tape of the form $\operatorname{INPUT}\left\langle\varepsilon_{0}, \varepsilon_{1}, \ldots, \varepsilon_{m-1}\right\rangle$. In this interval, the field

$$
\operatorname{INPUT}\left\langle\varepsilon_{0}, \varepsilon_{1}, \ldots, \varepsilon_{m-1}\right\rangle
$$

must be included in its entirety; moreover, it should be surrounded by at least one blank cell on both the left and the right. 
Some properties of models of the theory $T$ constructed from $\mathcal{M}$ are described by the following statement.

Lemma 6.1. Let $\varepsilon=\left\langle\varepsilon_{0}, \varepsilon_{1}, \ldots, \varepsilon_{m-1}\right\rangle, \varepsilon_{i} \in\{0,1\}, i<m$, be a sequence such that starting from the tape INPUT $\left\langle\varepsilon_{0}, \varepsilon_{1}, \ldots, \varepsilon_{m-1}\right\rangle$, the machine $\mathcal{M}$ enters a halt-state. Then the theory $T$ has a finite model $\mathfrak{M}$ in the form of a triangle shown in Fig. 3.5, whose class $J$ represents the field INPUT $\left\langle\varepsilon_{0}, \varepsilon_{1}, \ldots, \varepsilon_{m-1}\right\rangle$.

Proof. Consider the computation unfolded in time on the Turing machine $\mathcal{M}$ which starts from a tape of the form $\mathcal{S}=\operatorname{INPUT}\left\langle\varepsilon_{0}, \varepsilon_{1}, \ldots, \varepsilon_{m-1}\right\rangle$. It follows from our hypotheses that there is a halting point $h$ in this computation. Let $t$ be the $N$-successor of the point $h$; we clip the model $\operatorname{Compu}(\mathcal{M}, \mathcal{S})$ relative to this point $t$ in the form of a triangle as shown in Fig. 3.5. In this way, we obtain a finite model $\mathfrak{M}$. All predicates (3.3) of the signature of the model $\operatorname{Compu}\left(\mathcal{M}, \operatorname{INPUT}\left\langle\varepsilon_{0}, \varepsilon_{1}, \ldots, \varepsilon_{m-1}\right\rangle\right)$ are already defined in $\mathfrak{M}$ by rule (3.4). The other predicates of the signature $\sigma_{0}$ are defined in $\mathfrak{M}$ as follows:

$$
\begin{aligned}
H(x, y) & \Leftrightarrow x, y \text { are in the same row, } \\
D(x, y) & \Leftrightarrow \text { row }[y]_{H} \text { is the successor of row }[x]_{H}, \\
B L(x) & \Leftrightarrow x \text { lies on the left upper boundary of the triangle, } \\
B R(x) & \Leftrightarrow x \text { lies on the right upper boundary of the triangle, } \\
B(x) & \Leftrightarrow B L(x) \vee B R(x), \\
C T(x) & \Leftrightarrow x \text { is the top corner of the triangle, } \\
C L(x) & \Leftrightarrow x \text { is the left lower corner of the triangle, } \\
C R(x) & \Leftrightarrow x \text { is the right lower corner of the triangle. }
\end{aligned}
$$

One can easily check that all the axioms of the theory $T$ are true in our model $\mathfrak{M}$. Some difficulty is connected with checking the truth of Axiom $42^{\circ}$. For this purpose, notice that the machine can halt only after the head reaches a blank on the left boundary of the field INPUT. This guarantees the truth of Axiom $42^{\circ}$ in our model $\mathfrak{M}$.

Now our goal is to prove that this theory cannot have finite models other than those indicated in Lemma 6.1. For this purpose, we prove a sequence of properties of arbitrary finite models of the theory $T$.

Lemma 6.2. Let $\mathfrak{M}$ be a finite model of the theory $T$. Then its set $J(\mathfrak{M})$ represents a single $S$-chain segment, bounded on the left by the element $C L$, and on the right by the element $C R$.

Proof. Since the model $\mathfrak{M}$ is finite, its class $J(\mathfrak{M})$ is also finite. By Axioms $9^{\circ}$ and $10^{\circ}$, the class $J$ represents a unique $H$-class. By Axioms $21^{\circ}$, $22^{\circ}, 26^{\circ}$ and $27^{\circ}$ (existence of $S$-endpoints), Axioms $32^{\circ}$ and $33^{\circ}$ (connection), and Axioms $28^{\circ}$ and $29^{\circ}$ (restriction), we have that the class $J$ must consist of a unique $S$-chain segment with endpoints $C L$ and $C R$, and a finite number of 
$S$-cycles. The statement of Lemma 6.2 will be established if we can show that $S$-cycles in the class $J$ are impossible.

So, for the sake of a contradiction, assume there is an $S$-cycle $C$ in $J$. This cycle will have length $k \geqslant 2$ by virtue of Axiom $13^{\circ}$. By virtue of Axioms $21^{\circ}$ and $22^{\circ}$, there can be neither $B L$-elements nor $B R$-elements in $C$. Then, by virtue of Axioms $19^{\circ}$ and $20^{\circ}$, each element $a$ of $C$ must have an $N$-successor. By virtue of Axiom $16^{\circ}$, the set $C^{\prime}$ of the $N$-successors of elements of $C$ will again yield an $S$-cycle of the same length $k$. Since all elements in $C^{\prime}$ have $S$-successors and $S$-predecessors, by virtue of Axioms $21^{\circ}$ and $22^{\circ}$, there can be neither $B L$ elements nor $B R$-elements in $C^{\prime}$. Thus, the $N$-successors of the elements of $C^{\prime}$ will again yield an $S$-cycle $C^{\prime \prime}$ of the same length $k$, and this process continues. By our construction, if $C \subseteq J$, then by Axiom $30^{\circ}$, no elements of $C$ have $N$ predecessors, and thus the sequence of cycles $C, C^{\prime}, C^{\prime \prime} \ldots$ yields an infinite set of distinct elements, contradicting the finiteness of the model $\mathfrak{M}$. Thus, for the class $J(\mathfrak{M})$, there can be only one $S$-chain segment. The endpoints of this segment will satisfy the predicates $C L$ and $C R$ by virtue of Axioms $21^{\circ}, 22^{\circ}$, $32^{\circ}, 33^{\circ}$.

Lemma 6.3. Let $\mathfrak{M}$ be a finite model of the theory $T$. Then $\mathfrak{M}$ includes in its structure the triangle $\Delta(\mathfrak{M})$ of the form shown in Fig. 3.4, containing points $O, C L, C R$, and $C T$.

Proof. By Lemma 6.2, the class $J(\mathfrak{M})$ represents a finite $S$-chain segment, bounded on both sides by elements $C L$ and $C R$, and by Axioms $32^{\circ}$ and $33^{\circ}$, these endpoints also satisfy the predicates $B L$ and $B R$. Thus, in a given model $\mathfrak{M}$ there is an $S$-chain segment of the form

$$
\begin{aligned}
& B L\left(a_{0}\right), a_{0} S a_{1} S a_{2} S \ldots S a_{n-1} S a_{n}, B R\left(a_{n}\right), \\
& J\left(a_{i}\right), 0 \leqslant i \leqslant n .
\end{aligned}
$$

In the class $J$ of the model $\mathfrak{M}$ which includes only elements (6.1) by Lemma 6.2 , there is an element $O$, which, by Axioms $38^{\circ}$ and $36^{\circ}$, satisfies $Q(x)$, and by Axioms $45^{\circ}$ and $20^{\circ}, O$ cannot be the left or right endpoint of the chain (6.1). Thus, we obtain $n \geqslant 2$.

By Axioms $19^{\circ}$ and $20^{\circ}$, all elements of the chain (6.1), except for its two endpoint elements, must have $N$-successors, which, by Axiom $16^{\circ}$, again will form an $S$-chain. Thus, there are elements $b_{1}, \ldots, b_{n-1}$ such that

$$
\begin{aligned}
& B L\left(b_{1}\right), b_{1} S b_{2} S \ldots S b_{n-1}, B R\left(b_{n-1}\right), \\
& N\left(a_{i}, b_{i}\right), 1 \leqslant i \leqslant n-1,
\end{aligned}
$$

holds, where $B L\left(b_{1}\right)$ and $B R\left(b_{n-1}\right)$ are guaranteed by Axioms $23^{\circ}$ and $24^{\circ}$. If $b_{1}=b_{n-1}$, the required triangle $\Delta(\mathfrak{M})$ is completed. If $b_{1} \neq b_{n-1}$, then the case $S\left(b_{1}, b_{n-1}\right)$ is eliminated by Axiom $25^{\circ}$, and consequently in this situation we 
have $n \geqslant 4$. So in this case, the elements $b_{i}, 2 \leqslant i \leqslant n-2$, have $N$-successors $c_{i}$, which by Axiom $16^{\circ}$ again will yield an $S$-chain. Thus, for the new elements $c_{j}$ we are in a situation similar to (6.2), but with the length of the chain reduced by 2 . If $c_{2}=c_{n-2}$, then the triangle $\Delta(\mathfrak{M})$ is completed; otherwise, the process continues. Since the chain (6.1) is finite and the lengths of the successive chains decrease, this process will terminate, and the element, obtained at the last step of the process (which is the unique element in this $S$-chain) will be the top of the triangle, that is, it will satisfy $C T(x)$ by Axiom $31^{\circ}$.

In the triangle thus obtained, the left corner satisfies the predicate $C L$, the right corner satisfies $C R$, the upper corner satisfies $C T$, the bottom row satisfies $J$, the left-hand boundary satisfies $B L$, and the right-hand boundary satisfies $B R$. The dotted line for the machine head is determined by the predicate $Q(x)$, starting from the point $O$ of the class $J$, and by Axioms $47^{\circ}-49^{\circ}$, it can only end at the halting point of the machine $\mathcal{M}$, which is necessarily the $N$-predecessor of the upper corner $C T$ of the triangle $\Delta(\mathfrak{M})$.

Thus, as a result, in a given finite model $\mathfrak{M}$ of the theory $T$, a triangle $\Delta$ of the form shown in Fig. 3.4 can be found.

Lemma 6.4. Let $\mathfrak{M}$ be a finite model of the theory $T$. Then $\mathfrak{M}$ consists only of a single triangle $\Delta(\mathfrak{M})$ of the form shown in Fig. 3.4.

Proof. We remark first that the triangle $\Delta(\mathfrak{M})$, from the point of view of the successor function $D$ on $H$-classes, represents a $D$-chain segment of the form

$$
J\left(d_{0}\right),\left[d_{0}\right]_{H} D\left[d_{1}\right]_{H} D\left[d_{2}\right]_{H} D \ldots D\left[d_{n-1}\right]_{H} D\left[d_{n}\right]_{H}, C T\left(d_{n}\right),
$$

where the endpoint elements $d_{0}$ and $d_{n}$ are defined by Axioms $7^{\circ}$ and $9^{\circ}$. Now we consider an arbitrary element $a \in \mathfrak{M}$ and the $H$-class $[a]_{H}$ generated by $a$.

We will first show that the given class $[a]_{H}$ coincides with an $H$-class of the chain (6.3). By Axioms $26^{\circ}$ and $27^{\circ}$, the $H$-class $[a]_{H}$ contains elements $b$ and $c$ such that $B L(b)$ and $B R(c)$ hold. By Axioms $21^{\circ}$ and $22^{\circ}$, the element $b$ has no $S$-predecessor, and $c$ has no $S$-successor. By Axioms $28^{\circ}$ and $29^{\circ}$, elements of type $B L$ and $B R$ are unique in each $H$-class, therefore, all other elements in $[a]_{H}$ have $S$-predecessors and $S$-successors. From this, we obtain that the elements $b$ and $c$ must be connected by a finite $S$-chain of the form

$$
B L\left(e_{0}\right), b=e_{0} S e_{1} S e_{2} S \ldots S e_{n-1} S e_{n}=c, B R\left(e_{n}\right),
$$

since otherwise, the class $[a]_{H}$, and therefore the whole model $\mathfrak{M}$, would contain an infinite set of distinct elements, contradicting the finiteness of the model. Now it is necessary to perform standard manipulations with the chain (6.4). If $n=0$, then $b=c$, and consequently the element $b$ will satisfy $C T(x)$ by Axiom $31^{\circ}$; therefore, the $H$-class $[a]_{H}$ will coincide with $\left[d_{n}\right]_{H}$ by the uniqueness of the element $C T$. Now we consider the case $n>0$. The case $n=1$ is impossible by 
Axiom $25^{\circ}$, therefore we have $n \geqslant 2$. We consider the $N$-successors $e_{1}^{\prime}, \ldots, e_{n-1}^{\prime}$ of the elements $e_{1}, \ldots, e_{n-1}$, which exist by Axioms $19^{\circ}$ and $20^{\circ}$. By Axiom $16^{\circ}$, we have that these elements again form an $S$-chain of the form

$$
B L\left(e_{1}^{\prime}\right), e_{1}^{\prime} S e_{2}^{\prime} S \ldots S e_{n-1}^{\prime}, B R\left(e_{n-1}^{\prime}\right),
$$

where the truth of the predicates $B L$ and $B R$ at the endpoints $e_{1}^{\prime}$ and $e_{n-1}^{\prime}$ is guaranteed by Axioms $23^{\circ}$ and $24^{\circ}$. On the one hand, we have obtained a chain (6.5) of length 2 less than the length of the chain (6.4), and on the other hand, in view of Axiom $18^{\circ}$, the elements of the chain (6.5) are contained in an $H$-class, which is the $D$-successor of the class $[a]_{H}$, where our initial chain $(6.4)$ was formed. Repeating this process, we will eventually reach a chain consisting of a single element, which by Axiom $31^{\circ}$ will satisfy the predicate $C T$. Since such an element is unique by Axiom $8^{\circ}$, by virtue of (6.3), it must coincide with $d_{n}$. Thus, the class $[a]_{H}$ is at a finite distance (with respect to the successor function $D$ on $H$-chains) from the class $\left[d_{n}\right]_{H}$ in chain (6.3), therefore, the class $[a]_{H}$ must coincide with one of the $H$-classes of the chain (6.3).

Next, we show that $a \in \Delta(\mathfrak{M})$. For this, we consider the sequence of $N$ predecessors of the element $a$ of the form

$$
N\left(a_{1}, a\right), N\left(a_{2}, a_{1}\right), \ldots, N\left(a_{k}, a_{k-1}\right), \ldots
$$

Since, by Axiom $18^{\circ}$, passing to an $N$-predecessor means passing to the $D$ preceding $H$-class in the chain $(6.3)$, the class $\left[d_{0}\right]_{H}$ will eventually be reached in finitely many steps. By Axiom $9^{\circ}$, it does not have a $D$-predecessor, and consequently the corresponding element $a_{k} \in\left[d_{0}\right]_{H}$ in the chain (6.6) will not have an $N$-predecessor. It remains to note that $J\left(a_{k}\right)$ must hold, and consequently, by Lemma 6.2 , the element $a_{k}$ must be located on the $S$-chain segment representing the bottom row of the triangle $\Delta(\mathfrak{M})$. By the construction, the element $a$ is connected with $a_{k}$ by an $N$-chain, and $\Delta(\mathfrak{M})$ is closed with respect to $N$-successors. So, we have $a \in \Delta(\mathfrak{M})$ as required.

Lemma 6.5. Let $\mathfrak{M}$ be a finite model of the theory $T$. Then its class $J$ represents some starting situation $\mathcal{S} \in$ SpecST whose information field is included in it entirely; moreover, it is surrounded by at least one blank cell on both the left and the right.

PROOF. This property is immediately guaranteed by axioms $38^{\circ}-43^{\circ}$.

Lemma 6.6. Let $\mathfrak{M}$ be a finite model of the theory $T$. Then its class $J$ represents some starting situation $\operatorname{INPUT}\left\langle\varepsilon_{0}, \varepsilon_{1}, \ldots, \varepsilon_{m-1}\right\rangle$ with $m$ finite such that $\mathcal{M}$ has a halting point starting from $\operatorname{INPUT}\left\langle\varepsilon_{0}, \varepsilon_{1}, \ldots, \varepsilon_{m-1}\right\rangle$.

Proof. Since $\mathfrak{M}$ is a model of $T$, its class $J$ should represent a starting situation $\mathcal{S} \in \operatorname{SpecST}$ by Lemma 6.5. If $\mathcal{S}$ had a form different from the form $\operatorname{INPUT}\left\langle\varepsilon_{0}, \varepsilon_{1}, \ldots, \varepsilon_{m-1}\right\rangle$ for a finite sequence $\varepsilon=\left\langle\varepsilon_{0}, \varepsilon_{1}, \ldots, \varepsilon_{m-1}\right\rangle$, 
then $\mathcal{M}$ could not have a halting point by requirement (4.5), which contradicts the finiteness of the model since its top corner $C T(x)$ is directly connected to a halting state by Axioms $47^{\circ}-49^{\circ}$. Finally we obtain that the class $J$ of $\mathfrak{M}$ must represent a starting situation $\operatorname{INPUT}\left\langle\varepsilon_{0}, \varepsilon_{1}, \ldots, \varepsilon_{m-1}\right\rangle$ such that $\left\langle\varepsilon_{0}, \varepsilon_{1}, \ldots, \varepsilon_{m-1}\right\rangle \in \operatorname{Hlt}(\mathcal{M})$.

Lemma 6.7. Let $\varepsilon=\left\langle\varepsilon_{0}, \varepsilon_{1}, \ldots, \varepsilon_{m-1}\right\rangle$ be a finite sequence of zeros and ones such that $\mathcal{M}$ halts when starting from INPUT $\left\langle\varepsilon_{0}, \varepsilon_{1}, \ldots, \varepsilon_{m-1}\right\rangle$. Then the model $\mathfrak{M}$ of the theory $T$ whose class $J$ represents this starting situation $\operatorname{INPUT}\left\langle\varepsilon_{0}, \varepsilon_{1}, \ldots, \varepsilon_{m-1}\right\rangle$ is determined uniquely up to isomorphism.

Proof. The computation of $\mathcal{M}$ is uniquely determined by its program (which is fixed) and by its starting situation. This shows that the triangle clipping $\Delta$ from the computation $\operatorname{Compu}\left(\mathcal{M}, \operatorname{INPUT}\left\langle\varepsilon_{0}, \varepsilon_{1}, \ldots, \varepsilon_{m-1}\right\rangle\right)$ is defined uniquely, and by Lemmas 6.1 and 6.4, this triangle must occur in the finite model $\mathfrak{M}$ of the theory $T$; moreover, this model must be of the form $\mathfrak{M}=\Delta(\mathfrak{M})$.

We let $\mathfrak{M}\left\langle\varepsilon_{0}, \varepsilon_{1}, \ldots, \varepsilon_{m-1}\right\rangle, \varepsilon_{i} \in\{0,1\}$, denote the finite model of $T$ representing the starting situation INPUT $\left\langle\varepsilon_{0}, \varepsilon_{1}, \ldots, \varepsilon_{m-1}\right\rangle$. By the lemmas above, if this model exists, it is determined uniquely up to isomorphism by a set of input parameters $\varepsilon=\left\langle\varepsilon_{0}, \varepsilon_{1}, \ldots, \varepsilon_{m-1}\right\rangle$; moreover, this model exists if and only if the computation unfolded in time

$$
\operatorname{Compu}\left(\mathcal{M}, \operatorname{INPUT}\left\langle\varepsilon_{0}, \varepsilon_{1}, \ldots, \varepsilon_{m-1}\right\rangle\right)
$$

has a halting point. We call $m$ the weight of $\mathfrak{M}=\mathfrak{M}\left\langle\varepsilon_{0}, \varepsilon_{1}, \ldots, \varepsilon_{m-1}\right\rangle$, and denote it by weight $(\mathfrak{M})$.

In summary, we obtain the following description of the class of all finite models of the theory $T$, establishing our requirement (4.17):

Lemma 6.8. $\operatorname{ModFin}(T)=\operatorname{Mod}(T) \cap M_{\text {fin }}=\{\mathfrak{M}\langle\varepsilon\rangle \mid \varepsilon \in \operatorname{Hlt}(\mathcal{M})\}$.

Proof. Immediate by the above Lemmas 6.1-6.7.

\section{Geometric properties of models}

According to our construction, the models of the theory $T$ look like a flat net, generated by the successor relations $S$ and $N$. Also, in the model, there exists the successor function $D$ on the $H$-classes. Thus we can define in a natural way a distance function inside models of the theory, considering them as metric spaces.

Using the relations $S$ and $N$ given in a model $\mathfrak{M}$, we define (first-order definable) binary relations $N_{i}(x, y), S_{j}(x, y), S_{i} N_{j}(x, y)$, and $D_{i}(x, y)$, for arbitrary 
integer indices $i, j \in \mathbb{Z}$, as follows:

$$
\begin{aligned}
& S_{0}(x, y) \leftrightarrow(x=y), \\
& S_{1}(x, y) \leftrightarrow S(x, y), \\
& S_{-1}(x, y) \leftrightarrow S(y, x), \\
& S_{k}(x, y) \leftrightarrow\left(\exists z_{1} \ldots z_{k-1}\right)\left[S\left(x, z_{1}\right) \& S\left(z_{1}, z_{2}\right) \& \ldots \& S\left(z_{k-1}, y\right)\right], \quad k>1, \\
& S_{-k}(x, y) \leftrightarrow\left(\exists z_{1} \ldots z_{k-1}\right)\left[S\left(y, z_{1}\right) \& S\left(z_{1}, z_{2}\right) \& \ldots \& S\left(z_{k-1}, x\right)\right], \quad k>1, \\
& N_{0}(x, y) \leftrightarrow(x=y), \\
& N_{1}(x, y) \leftrightarrow N(x, y), \\
& N_{-1}(x, y) \leftrightarrow N(y, x), \\
& N_{k}(x, y) \leftrightarrow\left(\exists z_{1} \ldots z_{k-1}\right)\left[N\left(x, z_{1}\right) \& N\left(z_{1}, z_{2}\right) \& \ldots \& N\left(z_{k-1}, y\right)\right], k>1, \\
& N_{-k}(x, y) \leftrightarrow\left(\exists z_{1} \ldots z_{k-1}\right)\left[N\left(y, z_{1}\right) \& N\left(z_{1}, z_{2}\right) \& \ldots \& N\left(z_{k-1}, x\right)\right], k>1, \\
& S_{i} N_{j}(x, y) \leftrightarrow(\exists z)\left[S_{i}(x, z) \& N_{j}(z, y)\right] \vee(\exists w)\left[N_{j}(x, w) \& S_{i}(w, y)\right], \\
& D_{0}(x, y) \leftrightarrow H(x, y), \\
& D_{1}(x, y) \leftrightarrow D(x, y), \\
& D_{-1}(x, y) \leftrightarrow D(y, x), \\
& D_{k}(x, y) \leftrightarrow\left(\exists z_{1} \ldots z_{k-1}\right)\left[D\left(x, z_{1}\right) \& D\left(z_{1}, z_{2}\right) \& \ldots \& D\left(z_{k-1}, y\right)\right], k>1, \\
& D_{-k}(x, y) \leftrightarrow\left(\exists z_{1} \ldots z_{k-1}\right)\left[D\left(y, z_{1}\right) \& D\left(z_{1}, z_{2}\right) \& \ldots \& D\left(z_{k-1}, x\right)\right], k>1 .
\end{aligned}
$$

If $S_{k}(x, y)$ is true, we say that the element $y$ is the $k$-th $S$-successor of the element $x$, and the element $x$ is the $k$-th $S$-predecessor of the element $y$. The term $k$-th $N$-successor with $k \in \mathbb{Z}$ for the relation $N(x, y)$ is defined similarly.

Let $\mathfrak{M}$ be an arbitrary model of the theory $T$. If $a, b \in \mathfrak{M}$ and $D_{k}(a, b)$ holds for some $k \in \mathbb{Z}$, then we say that the distance inside the $D$-chain from $a$ to $b$ is equal to $|k|$, and denote this by

$$
\rho^{*}(a, b)=|k| .
$$

This function satisfies the following requirements:

$$
\begin{aligned}
& \rho^{*}(a, b) \geqslant 0, \\
& \rho^{*}(a, b)=0 \Leftrightarrow H(a, b), \\
& \rho^{*}(a, b) \leqslant \rho^{*}(a, c)+\rho^{*}(c, b) .
\end{aligned}
$$

A finite model $\mathfrak{M}$ together with the function $\rho^{*}$ forms a metric space on its $H$-classes (not on its elements). We will therefore call $\rho^{*}$ a quasi-distance.

Let $\mathfrak{M}$ be an arbitrary model of the theory $T$. If $a, b \in \mathfrak{M}$, and we have $S_{i} N_{j}(a, b), i, j \in \mathbb{Z}$, one can define a distance function between these two elements by the rule

$$
\rho(a, b)=|i|+|j| .
$$


In each finite model $\mathfrak{M}$ of the theory $T$, this distance $\rho(x, y)$ satisfies the following natural conditions for any $a, b, c$ :

$$
\begin{aligned}
& \rho(a, b) \geqslant 0, \\
& \rho(a, b)=0 \Leftrightarrow a=b, \\
& \rho(a, b) \leqslant \rho(a, c)+\rho(c, b) .
\end{aligned}
$$

Thus, a finite model $\mathfrak{M}$ together with the distance function $\rho$ gives a metric space. The relation

$$
\rho^{*}(a, b) \leqslant \rho(a, b)
$$

between the distance and the quasi-distance functions can be verified immediately.

The distance between two sets $X, Y \subseteq \mathfrak{M}$ is defined by

$$
\rho(X, Y)=\min \{\rho(a, b) \mid a \in X, b \in Y\}
$$

In the case of a one-element set $X$, we write $\rho(a, Y)$ instead of $\rho(\{a\}, Y)$.

Define the concept of a sphere of radius $r \geqslant 0$ with center $a \in \mathfrak{M}$ by

$$
\operatorname{Sp}(a, r)=\{x \in \mathfrak{M} \mid \rho(a, x) \leqslant r\} .
$$

One can show that $|\operatorname{Sp}(a, r)| \leqslant 2 r(r+1)+1$.

For $a \in \mathfrak{M}$ and $r \in \mathbb{N}$, we denote by $\tau(a, r)$ the isomorphism type of the model $\mathrm{Sp}^{*}(a, r)=\mathfrak{M} \uparrow \operatorname{Sp}(a, r)$. Thus, $\tau(a, r)=\tau(b, r)$ means that the spheres $\mathrm{Sp}^{*}(a, r)$ and $\mathrm{Sp}^{*}(b, r)$ considered together with the structure inherited from the model $\mathfrak{M}$ are isomorphic. It is obvious that if $\operatorname{Sp}^{*}(a, r)$ and $\operatorname{Sp}^{*}(b, r)$ are isomorphic, then the isomorphism $\mu$ between these submodels of $\mathfrak{M}$ is unique, and we have $\mu(a)=b$.

We call a set $X \subseteq \mathfrak{M}$ connected if for any elements $a, b \in X$, there is finite chain $a=c_{0}, c_{1}, \ldots, c_{n}=b$ such that $c_{i} \in X$, and $\rho\left(c_{i}, c_{i+1}\right)=1$ for all $i<n$. An elementary example of a connected set is a sphere of radius $r$ with center $a$.

For $X \subseteq \mathfrak{M}$, a set $Y \subseteq X$ is called a connected component of $X$ if $Y$ is a maximal connected subset in $X$. It is obvious that any set $X$ can be uniquely represented as the union of its connected components $X=Y_{1} \cup Y_{2} \cup \ldots \cup Y_{k}$; moreover, $\rho\left(Y_{i}, Y_{j}\right)>1$ for all $i, j$ with $i \neq j$, and the above decomposition is unique for a given $X$.

Lemma 7.1. Let $\mathfrak{M}$ and $\mathfrak{N}$ be models of the theory $T, X \subseteq \mathfrak{M}, Y \subseteq \mathfrak{N}$, and let $X=X_{1} \cup X_{2} \cup \ldots \cup X_{n}, Y=Y_{1} \cup Y_{2} \cup \ldots \cup Y_{n}$ be decompositions of these sets into their connected components (the number of connected components being the same for $X$ and $Y$ ). Also assume we are given bijective mappings $f_{i}: X_{i} \rightarrow Y_{i}, i=1,2, \ldots, n$. Then if the mappings $f_{i}$ are isomorphisms, and 
their union $f=f_{1} \cup f_{2} \cup \ldots \cup f_{k}$ preserves both $H(x, y)$ and $D(x, y)$, then $f$ is an isomorphism between $\mathfrak{M}\lceil X$ and $\mathfrak{N} \uparrow Y$.

Proof. In this situation, $f$ preserves all unary predicates of the signature $\sigma$ as well as the predicates $S$ and $N$ on the connected components. It remains to note that both binary predicates $S$ and $N$ fail on pairs of elements from different components, and consequently the map $f$ will be an isomorphism on the submodels of $\mathfrak{M}$ and $\mathfrak{N}$ given above.

Lemma 7.2. [Property of spheres of radius powers of 3] Let $a$ and $b$ be any elements of a model $\mathfrak{M}$ of the theory $T$. Then for arbitrary $k \in \mathbb{N}$, we have

$$
\operatorname{Sp}\left(a, 3^{k}\right) \cap \operatorname{Sp}\left(b, 3^{k}\right) \neq \varnothing \Leftrightarrow \operatorname{Sp}\left(a, 3^{k}\right) \subseteq \operatorname{Sp}\left(b, 3^{k+1}\right) .
$$

Proof. By direct calculation of the distances, using the triangle inequality for the distance function $\rho(x, y)$.
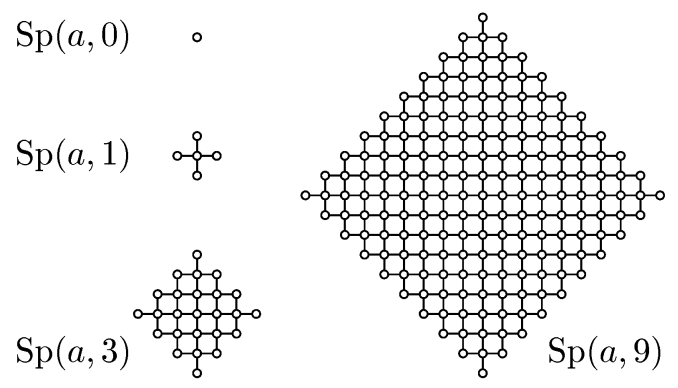

Fig. 7.1. Examples of spheres of exponent 3

The form of the spheres of radius powers of 3 for elements which are far from the boundaries of a model is shown in Fig. 7.1. The spheres of such radii will play a special role in the proof of our basic combinatorial statement.

Consider some simple but useful properties of the distance functions for a model $\mathfrak{M}$ of the theory $T$. We are interested in the distance to the left side $B L$ and the right side $B R$ of the triangle of this model. We say that two elements $a$ and $b$ are on the same horizontal line if we have $S_{n}(a, b)$ for some $n \in \mathbb{Z}$. Also we say that $a$ and $b$ are on the same vertical line, if we have $N_{n}(a, b)$ for some $n \in \mathbb{Z}$.

Lemma 7.3. Let $a, b \in \mathfrak{M}$ be on the same horizontal line; moreover, let $a$ be located to left of $b$. Then the following relations must hold:

$$
\begin{aligned}
& \rho(b, B L)=\rho(a, B L)+\rho(a, b), \\
& \rho(a, B R)=\rho(b, B R)+\rho(a, b) .
\end{aligned}
$$

Proof. Obvious. 
Lemma 7.4. Let $a, b \in \mathfrak{M}$ be on the same vertical line, and let $b$ be located above $a$. Then the following relations must hold:

$$
\begin{aligned}
& \rho(a, B L)=\rho(b, B L)+\rho(a, b), \\
& \rho(a, B R)=\rho(b, B R)+\rho(a, b) .
\end{aligned}
$$

Proof. Obvious.

Recall that the $Q$-line is the line traced by the motion of the head during the Turing machine computation.

Lemma 7.5. Let $a \in \mathfrak{M}$ be on the $Q$-line. Then, for arbitrary $n \in \mathbb{N}$, the following statements hold:

(a) if, after point a in $\mathfrak{M}$ (including a itself), there are $n$ (or more) points at which the machine head moves left, then $\rho(a, B L) \geqslant 2 n+1$.

(b) if, after point a in $\mathfrak{M}$ (including a itself), there are $n$ (or more) points at which the machine head moves right, then $\rho(a, B R) \geqslant 2 n+1$.

Proof. Obvious.

\section{General form of a model}

Now we pass to a more detailed investigation of the structure of the models of the theory $T$. We are mainly interested in the finite models of the theory $T$, which, as proved above, look like $\mathfrak{M}\langle\varepsilon\rangle$ for $\varepsilon \in \operatorname{Hlt}(\mathcal{M})$. Fig. 8.1, shows both the general motion of the head of the Turing machine $\mathcal{M}$ in a finite model (without the details of tape symbols and conditions), and also the computation areas inside the universal block.

According to the flow chart, a computation of the machine $\mathcal{M}$, represented in the model $\mathfrak{M}\langle\varepsilon\rangle$, proceeds as follows. In the initial phase, in the interval from (a) to the first entry into the universal block at point (d), it performs initialization, which installs the pointer $t$ at the beginning of the field INPUT, 


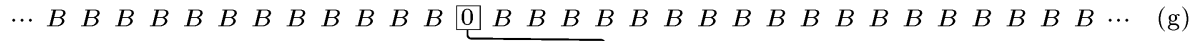

$\cdots B \quad B \quad B \begin{array}{llllllllllllllllllllllllllllll}B & 0 & 0 & 0 & 1 & 0 & 0 & 1 & 0 & B & 1 & 1 & 1 & B & B & B & B & B & B & B & B & B & B & B & B & B & B & B & \cdots & \text { (f) }\end{array}$ $\stackrel{\rightleftharpoons}{\rightleftharpoons}$

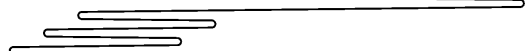

$\begin{array}{llllllllllllllllllllllllllllllllllll}\cdots & B & B & B & B & 0 & 0 & 0^{\prime} & 1 & 0 & 0 & 1 & 0 & B & 1 & 1 & 1 & B & 1 & 1 & 1 & B & 1 & 1 & 1 & 1 & 1 & 1 & 1 & B & B & B & \cdots & (\mathrm{e})\end{array}$ $U(2,2)$

$\begin{array}{lllllllllllllllllllllllllllllllllll}\cdots & B & B & B & B & 0 & 0 & 0^{\prime} & 1 & 0 & 0 & 1 & 0 & B & 1 & 1 & 1 & B & 1 & 1 & 1 & B & 1 & 1 & 1 & 1 & 1 & 1 & 1 & B & B & B & \cdots & \text { (d) }\end{array}$

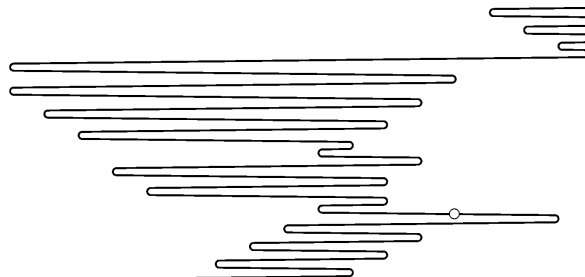

$\begin{array}{lllllllllllllllllllllllllllllllll}\cdots & B & B & B & B & 0 & 0 & 0^{\prime} & 1 & 0 & 0 & 1 & 0 & B & 1 & 1 & 1 & B & 1 & 1 & B & 1 & 1 & 1 & B & B & B & B & B & B & B & B & \cdots\end{array}$ $U(2,1)$

$\begin{array}{lllllllllllllllllllllllllllllllllll}\cdots & B & B & B & B & 0 & 0 & 0^{\prime} & 1 & 0 & 0 & 1 & 0 & B & 1 & 1 & 1 & B & 1 & 1 & B & 1 & 1 & 1 & B & B & B & B & B & B & B & B & \cdots & (\mathrm{d})\end{array}$

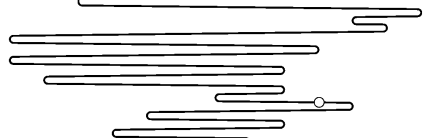

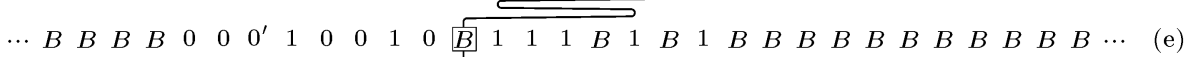
$U(2,0)$

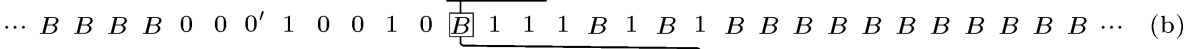

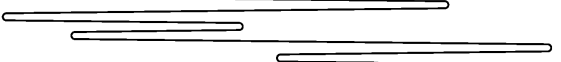

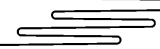

$\begin{array}{llllllllllllllllllllllllllllllllll}\cdots & B & B & B & B & 0 & 0 & 0 & 1 & 0 & 0^{\prime} & 1 & 0 & B & 1 & 1 & B & 1 & 1 & B & 1 & 1 & 1 & B & B & B & B & B & B & B & B & B & \cdots & \text { (e) }\end{array}$ $U(1,1)$

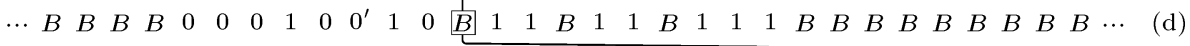

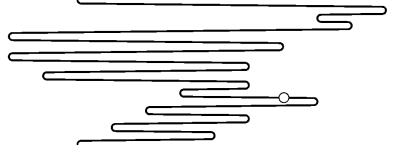

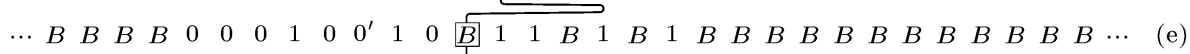
$U(1,0)$

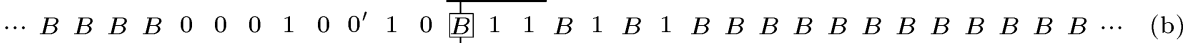

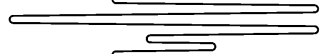

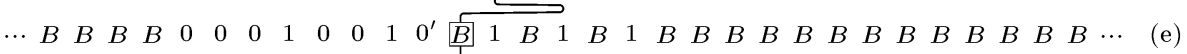
$U(0,0)$

$\begin{array}{lllllllllllllllllllllllllllllllllll}\cdots & B & B & B & B & 0 & 0 & 0 & 1 & 0 & 0 & 1 & 0^{\prime} & B & 1 & B & 1 & B & 1 & B & B & B & B & B & B & B & B & B & B & B & B & B & \cdots & \text { (b) }\end{array}$

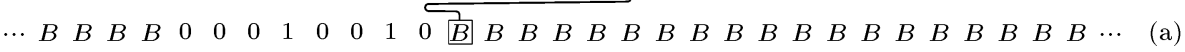

Fig. 8.1. Trace of Turing machine $\mathcal{M}$ with $\operatorname{INPUT}\langle 1,1\rangle$ 


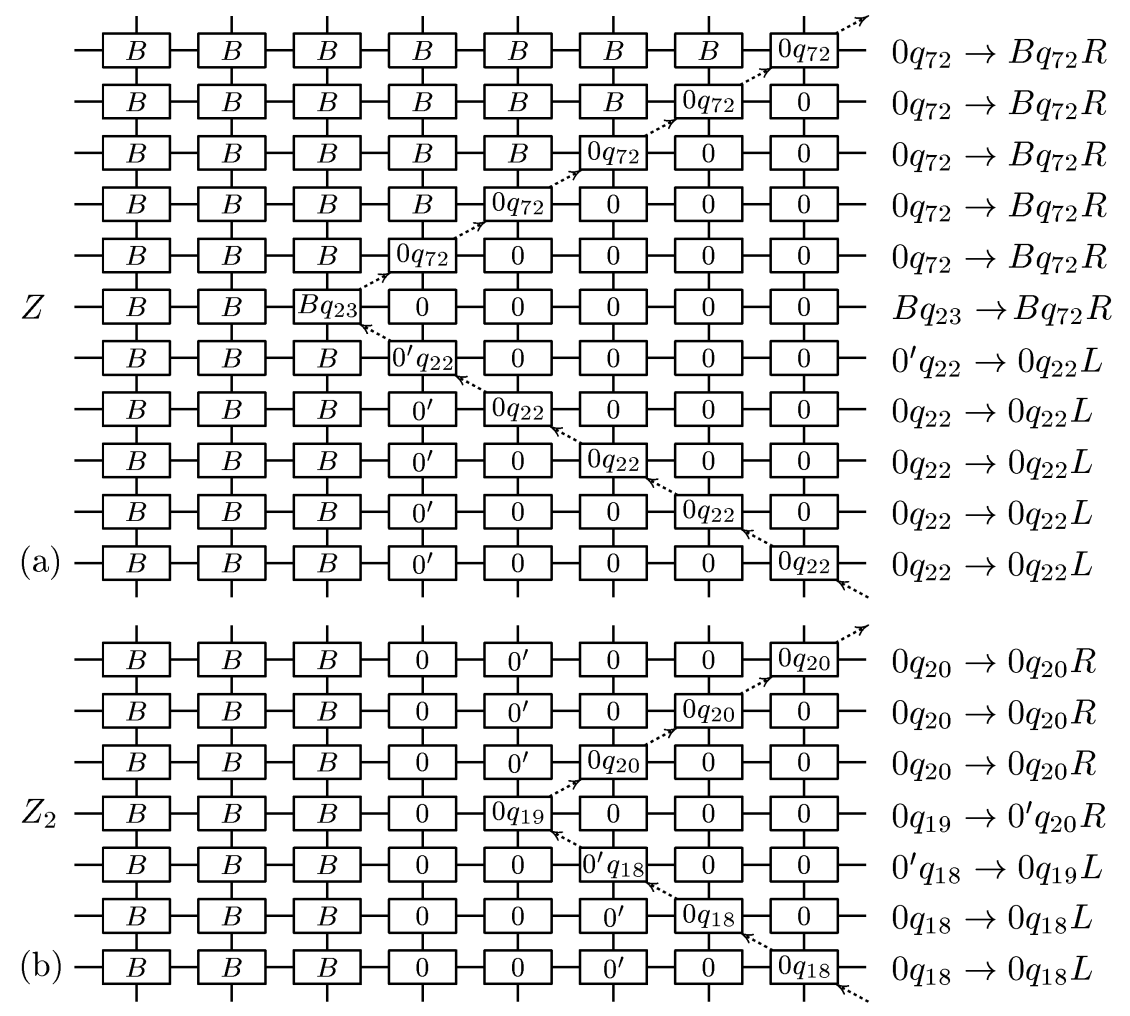

Fig. 8.2. Examples of fragments of the computation in a model

and also forms numerical cells $n, x$ and $e$ with their initial zero values. Then a global cycle begins during which we enter into the universal block for computations of the form $U(n, x)$. Note that Figure 8.1 shows stages (c) indicating the points where the program enters into the subroutine (c)-(d) before entering the universal block; they are marked just as circles on the $Q$-line. One can check that during the computation, from point (e) to point (c), the value of cell $x$ is increased by 1 , while the cell $e$ stays of the same value but is shifted by one cell to the right. According to the algorithm, in the model $\mathfrak{M}$ of the theory $T$ with weight $(\mathfrak{M})=m$, during the execution of the global cycle, the following series of calls of the universal block occurs

$$
\begin{aligned}
& U\left(0, e_{0}\right), U\left(1, e_{0}\right), U\left(1, e_{1}\right), \ldots, U\left(m, e_{0}\right), U\left(m, e_{1}\right), \ldots, U\left(m, e_{m}\right), \\
& \text { where } e_{x}=\operatorname{Nom}\left\langle\varepsilon_{0}, \varepsilon_{1}, \ldots, \varepsilon_{x-1}\right\rangle,
\end{aligned}
$$

exactly in this order (see Fig. 8.1), where we use the abbreviation (5.1).

When, at the next increase of the pointer $t$, a blank indicating the bound of the field INPUT is reached, control is passed to the completion procedure (i.e., 
the final part of the computation), which erases all tape information and finally enters the halt situation in the same cell at which the machine started. Thus the triangle of computation in a finite model $\mathfrak{M}$ of the theory $T$ is a symmetric figure relative to the vertical line through the starting point of the machine (i.e., the triangle is isosceles). Two examples of models at some particular points of their computations are depicted in Fig. 8.2. They can be used to demonstrate the concept of quasi-periodic computations as considered in Section 5.

Lemma 8.1. Let $m \in \mathbb{N}$ be given, and $\varepsilon \in \operatorname{Hlt}(\mathcal{M})$ be such that $|\varepsilon| \geqslant m$. Then all calls of the universal block of the form

$$
U(n, e), \quad n \leqslant m, e=\operatorname{Nom}\left\langle\varepsilon_{0}, \varepsilon_{1}, \ldots, \varepsilon_{x-1}\right\rangle, \quad 0 \leqslant x \leqslant n,
$$

are made in the model $\mathfrak{M}$.

PRoOF. Immediately from the properties of the algorithm $\mathcal{M}$ considered earlier in (5.12) and (8.1).

Consider the structure of the models at the beginning of a computation in a neighborhood of the starting point $O$.

Lemma 8.2. Let $\varepsilon, \varepsilon^{\prime} \in 2^{<\omega}, \varepsilon \prec \varepsilon^{\prime}$, and fix the models $\mathfrak{M}\langle\varepsilon\rangle$ and $\mathfrak{M}\left\langle\varepsilon^{\prime}\right\rangle$. Consider in the model $\mathfrak{M}\langle\varepsilon\rangle$ the area $\mathfrak{T}=\operatorname{Trpz}(\mathfrak{M}\langle\varepsilon\rangle)$, a region in the form of a trapezoid which is bounded below by the line J, from above by the horizontal line immediately preceding the moment of detection of the end of the field INPUT $\langle\varepsilon\rangle$ by the command $B q_{23} \rightarrow B q_{72} R$, on the left by the last zero cell in the field INPUT $\langle\varepsilon\rangle$, and on the right by the boundary BR of the triangle of the model $\mathfrak{M}\langle\varepsilon\rangle$, except for this line itself. Then there is an isomorphic embedding $\mu$ : $\mathfrak{M}\langle\varepsilon\rangle \uparrow \mathfrak{T} \rightarrow \mathfrak{M}\left\langle\varepsilon^{\prime}\right\rangle$.

Proof. The statement follows from the fact that the computations, beginning with the start situations $\operatorname{INPUT}\langle\varepsilon\rangle$ and $\operatorname{INPUT}\left\langle\varepsilon^{\prime}\right\rangle$ for $\varepsilon \prec \varepsilon^{\prime}$, proceed completely identically while the information read from the field INPUT is identical. The only tricky part of the proof consists in showing that, for the embedding $\mu$, the right side of the trapezoid $\mathfrak{T}$ will not be outside the right boundary of the model $\mathfrak{M}\left\langle\varepsilon^{\prime}\right\rangle$. This follows by comparing the distances from the left upper corner $a$ of the trapezoid $\mathfrak{T}$ (located on the $Q$-line, according to the construction) to the right boundary $B R$ of our model. The distance $r$ in $\mathfrak{M}\langle\varepsilon\rangle$ from $a$ to the boundary $B R$ equals

$$
2(|\operatorname{INPUT}\langle\varepsilon\rangle|+m+3)+1, \quad \text { where } m=\text { weight } \mathfrak{M}\langle\varepsilon\rangle,
$$

since erasing the area INPUT and the cell $n$ in the final part of the computations (the value of cell $n$ is equal to weight $\mathfrak{M}\langle\varepsilon\rangle$ at this stage) requires exactly this number of steps to the right (without the factor 2), and after that, the head only moves to the left before reaching the halt-state. On the other hand, in $\mathfrak{M}\left\langle\varepsilon^{\prime}\right\rangle$, the distance $r^{\prime}$, i.e., the distance from the element $\mu(a)$ to the right 
boundary $B R$, exceeds $r$ because, by Lemma 7.5 (b), in the model $\mathfrak{M}\left\langle\varepsilon^{\prime}\right\rangle$, when the computation passes into its final part, the fields INPUT and $n$ will be of larger lengths than they were in $\mathfrak{M}\langle\varepsilon\rangle$, and the head must move to the right past all of them.

The following technical fact holds:

Lemma 8.3. Let $\mathfrak{M}$ be an arbitrary finite model of the theory $T$. Then, in $\mathfrak{M}$, we have $\left.(\forall x)[B(x) \&\urcorner C T(x) \rightarrow A_{2}(x)\right]$.

Proof. In other words, Lemma 8.3 states that the tape symbols on the two boundaries of the triangle must be blanks. This holds because in the triangular clipping in Fig. 3.5, there can only be blanks on both sides of the point where the head halts since, by the specification of the algorithm, when the machine halts, the tape contains only blanks, except for the stopping point itself, which is marked with zero (see Fig. $5.3(\mathrm{~g})$ ).

\section{Description of the regions of a model}

The main purpose of the next three sections is to prove Statement (4.25). For this, we will first study and classify types of areas of models of the theory $T$, then classify "finite" 1-types realized in these models, and finally establish that there is an Ehrenfeucht property for these models which yields (4.25).

First of all, we fix some integer value

$$
s \geqslant 1
$$

(the number of steps in the Ehrenfeucht method), and we also fix some infinite sequence of zeros and ones

$$
\varepsilon^{*}=\left\langle\varepsilon_{0}, \varepsilon_{1}, \ldots, \varepsilon_{i}, \ldots\right\rangle \in \mathrm{Cons}^{*}, \quad \varepsilon_{i} \in\{0,1\}, i \in \omega .
$$

Recall that we can use any of properties (4.2)-(4.23) because they were established in Sections 5-8. Our goal is to find, for the given $s$ and $\varepsilon^{*}$, an integer bound $w^{*}=w^{*}\left(s, \varepsilon^{*}\right)$ such that

$$
\mathfrak{M}\left\langle\varepsilon^{\prime}\right\rangle \equiv_{s} \mathfrak{M}\left\langle\varepsilon^{\prime \prime}\right\rangle \text {, for all } \varepsilon^{\prime}, \varepsilon^{\prime \prime} \in \operatorname{Hlt}(\mathcal{M}) \text { with } \varepsilon^{\prime}, \varepsilon^{\prime \prime} \succcurlyeq \varepsilon^{*} \mid w^{*} \text {. }
$$

This will give us exactly the property (4.25) we need to prove.

We consider the class of all models of the following form, for a fixed $w>0$ :

$$
\mathfrak{M}\langle\varepsilon\rangle, \quad \varepsilon^{*} \mid w \preccurlyeq \varepsilon, \quad \varepsilon \in \operatorname{Hlt}(\mathfrak{M}) .
$$

The class of models (9.4) is infinite because all models of the form $\mathfrak{M}\left\langle\varepsilon^{*} \mid n\right\rangle$, $n \geqslant w$ exist by virtue of (4.18). We will enlarge the value of $w$ so that all the 
models of this class become more and more similar, until we reach some finite value of $w$ for which they are all $\equiv_{s}$-equivalent. As a result, we obtain the desired property (9.3).

All the work in the next three sections depends on the values of $s$ and $\varepsilon^{*}$ chosen in (9.1) and (9.2). For the sake of brevity, we often suppress these parameters, assuming them to be given by context.

Starting with $s$ from above, we define the following auxiliary parameters

$$
\begin{aligned}
& s^{+}=2 \cdot 3^{s-1}, \\
& s^{*}=s \cdot 2 \cdot 3^{s-1}=s \cdot s^{+}+1 .
\end{aligned}
$$

These parameters will play an important role in the later combinatorial computations. The parameter $s^{+}$describes the maximum possible space covered by a sphere of radius $3^{s-1}$ on a single $H$-class. Notice that $s^{+} \geqslant 2$ and $s^{*} \geqslant 3$ by (9.1).

The basic property of $s^{*}$ is as follows.

Lemma 9.1 [ Lemma on $s^{*}$-saturation] Fix $s$ and let $s^{*}$ be defined from $s$ by (9.5). Consider a finite model $\mathfrak{M}$ of the theory $T$. Then we have the following properties:

(a) If $a \in \mathfrak{M}, Z \subseteq[a]_{H},|Z| \geqslant s^{*}$, then for any $s$ elements $a_{1}, a_{2}, \ldots, a_{s}$ of the model $\mathfrak{M}$, we have

$$
Z \nsubseteq \operatorname{Sp}\left(a_{1}, 3^{s-1}\right) \cup \operatorname{Sp}\left(a_{2}, 3^{s-1}\right) \cup \ldots \cup \operatorname{Sp}\left(a_{s}, 3^{s-1}\right) .
$$

(b) If $Z \subseteq \mathfrak{M},(\forall x, y \in Z)[(x \neq y) \rightarrow\urcorner H(x, y)],|Z| \geqslant s^{*}$, then for any $s$ elements $a_{1}, a_{2}, \ldots, a_{s}$ of the model $\mathfrak{M}$, there is an element $b \in Z$ such that $\rho^{*}\left(a_{i}, b\right)>3^{s-1}$ for all $i \in\{1,2, \ldots, s\}$.

Proof. (a) Each of the sets $\operatorname{Sp}\left(a_{i}, 3^{s-1}\right)$ can contain no more than $s^{+}$ elements from $Z$. Since there are only $s$ such sets, they can cover no more than $s \cdot s^{+}=s^{*}-1$ elements of $Z$. But $Z$ contains $s^{*}$ or more elements, yielding (a) as desired.

(b) The proof of this statement is similar, with $H$-classes and the $D$-successor function defined on them, instead of separate elements.

To investigate elementary properties of models of the theory $T$, we need a general description of various areas of an arbitrary model $\mathfrak{M}$ of this theory, which is schematically represented in Fig. 9.1. The vertical and horizontal lines denote $N$-chains and $S$-chains, respectively, and all diagonal lines are assumed to lie at a $45^{\circ}$ degree angle with respect to the grid structure of the model.

By $B L$ and $B R$ we denote the left and right boundaries of the triangle $\Delta(\mathfrak{M})$. The letters $L$ and $R$ denote the left and right corners of the triangle, while $H$ 
denotes the halting-point of the computation represented in the model. By our design, the point $H$ is located in a neighborhood of the top $C T$ of the triangle $\Delta(\mathfrak{M})$. By $O$ we denote the start point of the Turing machine, which lies on the base $J$ of the triangle. The algorithm ensures that the cell scanned by the machine when the machine halts coincides with the cell scanned when the machine starts; therefore, the line $\mathrm{OH}$ is vertical, and it is an axis of symmetry for the whole triangle.

By (f), a horizontal line is denoted representing the point at which the machine detects the end of the field INPUT and passes to the final part of the computation. The notation (f) is also used in the flow chart and in the program of the machine $\mathcal{M}$. The position of the head on the line (f) is denoted by $Z$, while the point of intersection of the line (f) with the line $\mathrm{OH}$ is denoted by $P$. The vertical line $Z S$ is a boundary of the field INPUT. To the left of it, including the line $Z S$ itself, a field of only blanks is located. The line $Z E-E H$ represents the motion of the head in the final part of the computation. The point $C$ is the perpendicular projection of $E$ onto the line (f), the line $C D$ is the diagonal line, passing through the point $C$ parallel to the right boundary $B R$ of the triangle $\Delta(\mathfrak{M})$.

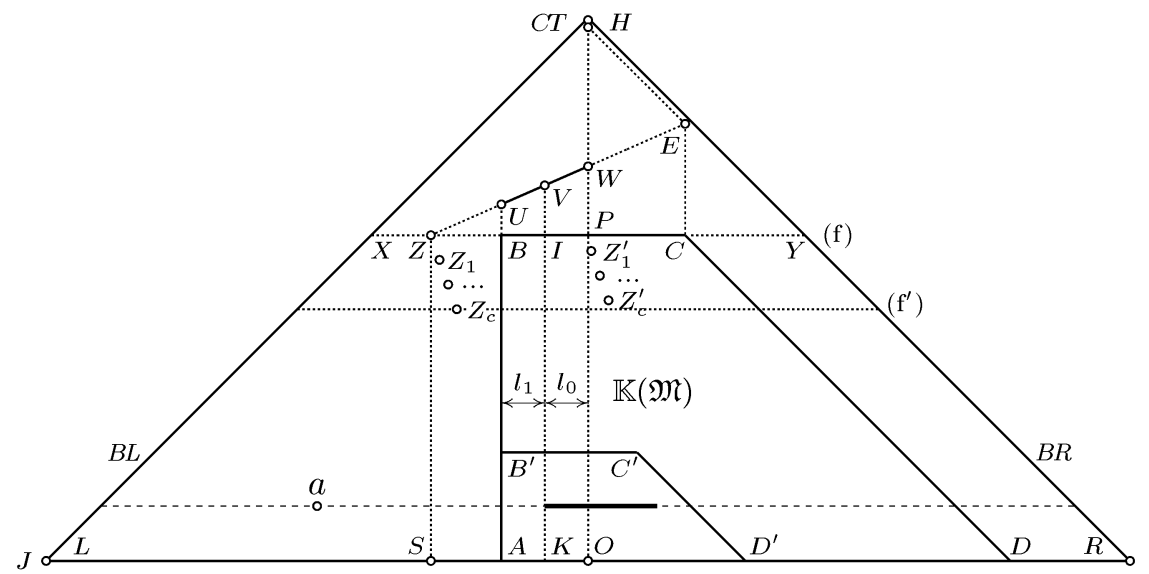

Fig. 9.1. Special regions and points of a model

By $Z_{i}, 1 \leqslant i \leqslant s^{+}$, we denote the sequence of points of a model which are in state $0 q_{19}$ as the Turing machine head approaches the left end of the field INPUT (and moves the symbol $0^{\prime}$ ) during the quasi-periodic computation, while by $Z_{i}^{\prime}, 1 \leqslant i \leqslant s^{+}$, we denote the points in state $1^{\prime} q_{20}$ located on the right sides of the same quasi-periodical computation. More precisely, the point $Z_{i}$ is the point in state $0 q_{19}$, at a distance $i$ from the field of blanks at the left, while $Z_{i}^{\prime}$ is the point in state $1^{\prime} q_{20}$, at a distance $i$ to the right from the line $O H$. The point $Z$ will be denoted by $Z_{0}$. For example, in Fig. 8.2, the form of the model in the neighborhood of the points $Z$ and $Z_{2}$ is shown. The location of the points $Z_{i}$ 
in the model can be seen in Fig. 9.1 and the positions of the points $Z_{i}^{\prime}$ are also shown.

We denote by $\left(\mathrm{f}^{\prime}\right)$ the horizontal line in a model passing through the point $Z_{c}$ with $c=s^{+}$, which is schematically shown in Fig. 9.1.

The area OPZS represents the field INPUT in the model $\mathfrak{M}$ from the start of the algorithm until condition (f) is reached. Inside the limits of this rectangle, we have the information $\varepsilon_{i}$ as vertical strips (actually, the strips $\varepsilon_{i}$ are in a larger region $O W Z S$ ), and in some parts of this area, we also have lines denoting the movement of the head as it moves the pointer $t$ and the marker $0^{\prime}$ as in the subroutine (c)-(d) (see Section 5). Thus, we see that the area OPZS has a rather simple form.

Consider the following integer-valued function

$$
E(k)=\frac{1}{2}(k+1)(k+4), k \in \mathbb{N},
$$

which was already applied in Section 4 . For $k<m$, the vertical strip containing the $\varepsilon_{k}$ cell is located at a distance $E(k)$ to the left of the line $O P$.

Recall the function $f(x)$ from Lemma 4.1 which satisfied conditions (4.20) through (4.23). We introduce the following three integer-valued parameters:

$$
\begin{aligned}
& l_{0}=E\left(s^{+}-2\right), \quad l_{1}=E(p)-l_{0}, \\
& w_{0}=\max \left\{p+1,1.5 \cdot s^{*}+s^{+}, 4 s^{+}\right\}, \\
& \text {where } \\
& p=(\mu t)\left[f(t) \geqslant s^{*}+s^{+}+6 \text { and } E(t)>l_{0}\right] .
\end{aligned}
$$

Property (4.21) of the function $f$ shows that the parameter $p$ in (9.8) is well defined. Obviously, we have $l_{0}>0$ and $l_{1}>0$. Moreover, the values of $l_{0}$ and $l_{1}$ depend just on the parameter $s$, but they do not depend on the choice of $\varepsilon^{*}$ in (9.2).

By $K I$, we denote the vertical line at a distance $l_{0}$ to the left of the line $O P$, while by $A B$, we denote the vertical line at a distance $l_{0}+l_{1}$ to the left of the line $O P$, where the values of the parameters $l_{0}$ and $l_{1}$ are given in (9.6). The line $A B$ is considered as undefined if it is outside of the rectangle $O P Z S$ or coincides with the line $S Z$.

Consider the following class of models

$$
\mathfrak{M}\langle\varepsilon\rangle, \quad \varepsilon^{*} \mid w_{0} \preccurlyeq \varepsilon, \quad \varepsilon \in \operatorname{Hlt}(\mathcal{M}) .
$$

Lemma 9.2. Both the line $A B$ and the rectangle $O P B A$ are defined in any model of the class (9.9).

Proof. By (9.6), the line $A B$ passes at a distance $l_{0}+l_{1}=E(p)$ of the line $O P$, while we have weight $(\mathfrak{M}) \geqslant w_{0}>p$ for all models of the class (9.9). 
Thus, the scheme presented in Fig. 9.1 is applicable to any model $\mathfrak{M}$ of the class (9.9).

Lemma 9.3. In any model $\mathfrak{M}$ of the class (9.9), the lines $K I$ and $A B$ pass along some strips $\varepsilon_{u}$ and $\varepsilon_{v}$.

Proof. By (9.6), we have $l_{0}=E\left(s^{+}-2\right)$ with $s^{+} \geqslant 2$, and $l_{0}+l_{1}=E(p)$. So, $l_{0}$ and $l_{1}+l_{0}$ are in the range of $E$. This gives the necessary property by the definition of the function $E(x)$.

Lemma 9.4. In the rectangle OPIK (including its left bound), there are $s^{+}-1$ strips of $\varepsilon_{i}$ cells.

Proof. By inspection.

Lemma 9.5. The distance between any two neighboring strips $\varepsilon_{i+1}$ and $\varepsilon_{i}$ in the rectangle KIZS is more than $s^{+}$.

Proof. By the choice of the parameter $l_{0}$ in (9.6), all neighboring pairs of strips located at a distance $\leqslant s^{+}$from each other are covered by the rectangle $O P I K$. This gives the desired property.

Lemma 9.6. In the rectangle KIBA there are more than $s^{*}+6$ strips $\varepsilon_{i}$ having the value $\varepsilon_{i}=0$, and more than $s^{*}+6$ strips $\varepsilon_{j}$ having the value $\varepsilon_{j}=1$.

Proof. We have $l_{0}+l_{1}=E(p)$ by the choice of parameters in (9.6), while by virtue of properties (4.22) and (4.23), the value of $p$ in (9.8) provides that in the rectangle $O P B A$ there are at least $f(p)=s^{*}+s^{+}+6$ strips $\varepsilon_{i}$ with value $\varepsilon_{i}=0$ and at least $f(p)$ strips $\varepsilon_{j}$ with value $\varepsilon_{j}=1$. But by Lemma 9.4, the rectangle OPIK together with its bounds covers just $s^{+}-1$ strips (of any kind). This gives the statements of Lemma 9.6.

Lemma 9.7. In any model $\mathfrak{M}$ of the class (9.9), the area LXZS of the model consists of only blanks.

Proof. Since, by the specification of the algorithm, the head of the Turing machine does not move to the left of the boundary of the field INPUT, except for the point $Z$ only, no other $Q$-points are present here. Therefore, the blanks present at the start of the computation will all remain blanks due to Axiom $46^{\circ}$.

Lemma 9.8. In any model $\mathfrak{M}$ of the class (9.9), the area DCER consists of only blanks.

Proof. At the stage (f), the head is at the point $Z$, so there cannot be other $Q$-points, except for $E$, in the area $D C E R$. Therefore all symbols here are preserved by Axiom $46^{\circ}$. Then, by Lemma 8.3, in the area DCER there cannot be symbols other than blanks.

Lemma 9.9. In any model $\mathfrak{M}$ of the class (9.9), the area $Z E C$ represents the information shown in Fig. $5.3(\mathrm{f})$, which is erased as the head moves along 
the line $Z E$.

Proof. The area $Z E C$ is generated by the situation $B q_{23}$ at the point $Z$ where we switch to the final part of computation.

Lemma 9.10. In any model $\mathfrak{M}$ of the class (9.9), the area XZEH has the following form: $W H$ is a line marked by symbols $0, E H$ is the line of head movement in a field of blanks (at a distance 1 from the boundary BR), all remaining points of area XZEH are blanks.

Proof. The area $X Y H$ is generated by the situation $B q_{23}$ due to the command $B q_{23} \rightarrow B q_{72} R$, and consequent passage to the final part of computation. This computation determines the areas specified in the statement of Lemma 9.10 .

Lemma 9.11. In any model $\mathfrak{M}$ of the class $(9.9)$ with weight $(\mathfrak{M})=m$, we have $\rho(E, H)=2 m+4$, and $\rho^{*}(E, H)=m+2$.

Proof. Both the distance and quasidistance can be easily counted by the scheme in Fig. 8.1, where the last head movement directly corresponds to the points $E$ and $H$ as well as to the cell $n$ which at this stage has the value weight $(\mathfrak{M})$. One can use some details in Fig 3.5 as well.

Lemma 9.12. In any model $\mathfrak{M}$ of the class (9.9) with weight $(\mathfrak{M})=m$, we have

$$
\rho(Z, X)=\rho(Z E, B L)=\rho(E, H)+1=2 m+5>s^{*}+s^{+} .
$$

Proof. One can see that in a model, the two lines $B L$ and $Z E$ are in fact parallel to each other. This gives all the equalities by virtue of Lemma 9.11. The last inequality is provided by (9.7) and (9.9).

Lemma 9.13. In any model $\mathfrak{M}$ of the class (9.9), we have

(a) $\rho^{*}(W, E)>s^{*}+s^{+}$,

(b) $\rho^{*}(E, H)>s^{*}+0.5 \cdot s^{+}+0.5 \cdot\left(s^{*}+s^{+}\right)$.

Proof. By (9.7) and Lemma 9.11, for a model $\mathfrak{M}$ of the class (9.9) with weight $(\mathfrak{M})=m$, we have $\rho^{*}(W, E)=\rho^{*}(E, H)=m+2>w_{0} \geqslant 1.5 s^{*}+s^{+}$. This gives the necessary inequalities.

Lemma 9.14. In any model $\mathfrak{M}$ of the class (9.9), we have

(a) $\rho(b, B L)>s^{*}+s^{+}$, for all $b \in Z S$.

(b) $\rho(c, B R)>s^{*}+s^{+}$, for all $c \in C D$.

Proof. Part (a) is a consequence of Lemma 9.12 and Lemma 7.4. As for (b), we have $\rho(C, Y)=\rho(C, E)+1>\rho^{*}(W, E)=\rho^{*}(E, H)=m+2>s^{*}+s^{+}$, where $m=$ weight $(\mathfrak{M})$ and the last inequality is provided by (9.7) and (9.9).

Lemma 9.15. Let $c=s^{+}=2 \cdot 3^{s-1}$. In any model $\mathfrak{M}$ of the class (9.9), all special points $Z_{1}, Z_{2}, \ldots, Z_{c}$ exist and are located in the region ABZS, while the 
points $Z_{1}^{\prime}, Z_{2}^{\prime}, \ldots, Z_{c}^{\prime}$ exist and are located in the region $O P C D$ of the model $\mathfrak{M}$.

Proof. Let $\mathfrak{M}$ be a model of the class (9.9). Since weight $(\mathfrak{M}) \geqslant w_{0}>s^{+}+1$ by (9.7), we see that the distance between the line $Z S$ and the strip $\varepsilon_{i}$ in the rectangle $O P Z S$ which is the closest to $Z S$ is more than $s^{+}+1$. Since the points $Z_{i}$ and $Z_{i}^{\prime}$ are the bounding points of a quasi-periodic computation (see Fig. 8.1), this means that the horizontal components of distances between $Z_{i}$ and $Z_{i+1}$ as well as between $Z_{i}^{\prime}$ and $Z_{i+1}^{\prime}$ are equal to 1 . This immediately gives the property stated in Lemma 9.15 .

Lemma 9.16. For any $i \in\left\{s^{+}, \ldots, 2,1\right\}$, the movement of the head from $Z_{i}$ to $Z_{i}^{\prime}$ and the movement of the head from $Z_{i}^{\prime}$ to $Z_{i-1}$ in the rectangle KIZS pass through at least $s^{*}+2$ strips $\varepsilon_{i}$ with the value $\varepsilon_{i}=0$, and through at least $s^{*}+2$ strips $\varepsilon_{j}$ with the value $\varepsilon_{j}=1$.

Proof. This follows directly from Lemma 9.6.

For a model $\mathfrak{M}$ of the class $(9.9)$, we denote by $\mathbb{K}(\mathfrak{M})$ the submodel of $\mathfrak{M}$ restricted to the area $S Z C D$, excluding its boundary lines $S Z$ and $Z C$. We call $\mathbb{K}(\mathfrak{M})$ the kernel of the model $\mathfrak{M}$. According to our construction, the kernel of the model can contain some complicated fragments (taking into account the arbitrary program of the kernel part of the universal block); however, the parts surrounding the kernel are rather simple as described in Lemmas 9.2-9.10. This fact provides the technical tools for showing that Statement (4.25) holds.

We study some properties of the kernel.

Lemma 9.17. Let $\mathfrak{M}\left\langle\varepsilon^{\prime}\right\rangle$ and $\mathfrak{M}\left\langle\varepsilon^{\prime \prime}\right\rangle$ be two models of the theory $T$ such that $\varepsilon^{\prime \prime} \succcurlyeq \varepsilon^{\prime} \succcurlyeq \varepsilon^{*} \mid w_{0}$. Then there is an isomorphic embedding $\mu: \mathbb{K}\left(\mathfrak{M}\left\langle\varepsilon^{\prime}\right\rangle\right) \rightarrow$ $\mathbb{K}\left(\mathfrak{M}\left\langle\varepsilon^{\prime \prime}\right\rangle\right)$, and this embedding is unique.

Proof. This lemma follows from Lemma 8.2 about monotonicity, which gives an isomorphic embedding for a larger area than is required. The uniqueness of the embedding follows directly from the uniqueness of the point $O$, and the rigidity of the frame, formed by the two successor relations $S$ and $N$.

Now we consider in more detail the relative position of $\mathbb{K}\left(\mathfrak{M}\left\langle\varepsilon^{\prime \prime}\right\rangle\right)$ and the image of $\mathbb{K}\left(\mathfrak{M}\left\langle\varepsilon^{\prime}\right\rangle\right)$. Let the triangle in Fig. 9.1 represent $\mathfrak{M}\left\langle\varepsilon^{\prime \prime}\right\rangle$. In the following lemma and in later sections, we will refer to the trapezoid $A B^{\prime} C^{\prime} D^{\prime}$ as representing the image of $\mathbb{K}\left(\mathfrak{M}\left\langle\varepsilon^{\prime}\right\rangle\right)$ without reference to $l_{0}$ or $l_{1}$. By this we mean to ignore the labels of $l_{0}$ and $l_{1}$ in Fig. 9.1 and view $A B^{\prime} C^{\prime} D^{\prime}$ as an arbitrary trapezoid inside the model which happens to be the image of $\mathbb{K}\left(\mathfrak{M}\left\langle\varepsilon^{\prime}\right\rangle\right)$.

Lemma 9.18. In any model $\mathfrak{M}$ of the class (9.9), all calls of the universal block are located in its kernel $\mathbb{K}(\mathfrak{M})$ below stage $\left(\mathrm{f}^{\prime}\right)$.

Proof. The point $Z$ is the beginning of the final part of the computation. Therefore, all calls of the universal block are located below stage (f). Furthermore, the part (f')-(f) represents a number of head movements during the last 
operation $t:=t+n$, which detects the end of field INPUT. So, all calls of the universal block should be in the kernel below stage (f').

Lemma 9.19. Whenever the universal block $U(n, e)$ is called in a model from the class (9.9) with input parameter $0 \leqslant x \leqslant 4 s^{+}$, then we have $e=$ $\operatorname{Nom}\left\langle\varepsilon_{0}, \varepsilon_{1}, \ldots, \varepsilon_{x-1}\right\rangle$, where all bit cells $\varepsilon_{i}$ used here are taken from the sequence $(9.2)$.

Proof. The value of the parameter $w_{0}$ is large enough by (9.7) (namely, $\left.w_{0} \geqslant 4 s^{+}\right)$, to ensure the truth of Lemma 9.19.

\section{Combinatorics of local and horizontal types}

In this section, we investigate the possible types of local areas of models of the theory $T$. We remark that whenever we write $\mathfrak{M}\left\langle\varepsilon^{\prime}\right\rangle$ or $\mathfrak{M}\left\langle\varepsilon^{\prime \prime}\right\rangle$ here or in the following, we tacitly assume that these finite models exist (i.e., that the corresponding Turing machine computations halt).

We let $\tau(a, r), a \in \mathfrak{M}$, denote the type of an element $a$ within of a sphere of radius $r$ with center $a$. We call this type local. For $\tau(a, r)$, it is possible to construct a formula $\theta(x)$ in one free variable $x$ which represents the atomic diagram of the $r$-neighborhood of this element $a$. Thus, in $\mathfrak{M}$, the formula $\theta(a)$ is true, and for any element $b$ from this or any other model, $\theta(b)$ is true if and only if $\tau(a, r)=\tau(b, r)$ holds. In this case, we say that the formula $\theta(x)$ represents the type $\tau(a, r)$. By $T Y(r)$ we denote the set of all formulas of the form $\theta(x)$, representing all possible local types of radius $r$ for different models of the theory $T$. It is obvious that the set $T Y(r)$ is finite for any $r \in \mathbb{N}$.

Note that the type of a given radius uniquely determines the existence of all elements in the neighborhood:

Lemma 10.1. Let $i, j \in \mathbb{Z}$ and $r \in \mathbb{N}$ be given such that $|i|+|j| \leqslant r$. Let $a \in \mathfrak{M}\left\langle\varepsilon^{\prime}\right\rangle$ and $b \in \mathfrak{M}\left\langle\varepsilon^{\prime \prime}\right\rangle$ for $\varepsilon^{\prime}, \varepsilon^{\prime \prime} \in 2^{<\omega}$. Then it follows from $\tau(a, r)=\tau(b, r)$ that $(\exists x) S_{i} N_{j}(a, x) \Leftrightarrow(\exists y) S_{i} N_{j}(b, y)$.

PROOF. Immediate by the definition of local type.

It is easy to see that the type of a given radius determines the type for any smaller radius.

Lemma 10.2. Fix $r^{\prime}, r \in \mathbb{N}$ such that $r^{\prime} \leqslant r$. Then for any two elements $a \in \mathfrak{M}\left\langle\varepsilon^{\prime}\right\rangle$ and $b \in \mathfrak{M}\left\langle\varepsilon^{\prime \prime}\right\rangle$ for $\varepsilon^{\prime}, \varepsilon^{\prime \prime} \in 2^{<\omega}, \tau(a, r)=\tau(b, r)$ implies $\tau\left(a, r^{\prime}\right)=$ $\tau\left(b, r^{\prime}\right)$.

ProOF. By inspection.

A local type of a given radius uniquely determines the type of a smaller radius not only of the same element, but also for some elements of its sphere. 
Lemma 10.3. Fix $i, j \in \mathbb{Z}$ and $k \in \mathbb{N}$ such that $|i|+|j| \leqslant 2 \cdot 3^{k-2}$. Also fix arbitrary elements $a, a^{\prime} \in \mathfrak{M}\left\langle\varepsilon^{\prime}\right\rangle$ and $b, b^{\prime} \in \mathfrak{M}\left\langle\varepsilon^{\prime \prime}\right\rangle$ for $\varepsilon^{\prime}, \varepsilon^{\prime \prime} \in 2^{<\omega}$ such that $S_{i} N_{j}\left(a, a^{\prime}\right)$ and $S_{i} N_{j}\left(b, b^{\prime}\right)$ hold. Then $\tau\left(a, 3^{k-1}\right)=\tau\left(b, 3^{k-1}\right)$ implies $\tau\left(a^{\prime}, 3^{k-2}\right)=\tau\left(b^{\prime}, 3^{k-2}\right)$.

Proof. Immediate by Lemma 7.2, given that the type declarations of $a$ and $b$ contain the complete type declarations of $a^{\prime}$ and $b^{\prime}$ since the spheres of $a$ and $b$ completely cover the spheres of $a^{\prime}$ and $b^{\prime}$, respectively.

Now we study types of $H$-classes of models of the theory $T$.

Let $r \in \mathbb{N}, t \in \mathbb{N}$, and fix an arbitrary function $h: T Y(r) \rightarrow \mathbb{N}$. We construct the following set of formulas in one free variable $x$ :

$$
\begin{aligned}
\Sigma(x)= & \left\{\left(\exists^{k} y\right)(H(x, y) \& \theta(y)) \mid \theta \in T Y(r), h(\theta)=k<t\right\} \cup \\
& \left\{\left(\exists^{\geqslant t} y\right)(H(x, y) \& \theta(y)) \mid \theta \in T Y(r), h(\theta) \geqslant t\right\} .
\end{aligned}
$$

We call the conjunction $\varphi(x)$ of all formulas in $\Sigma(x)$ the formula representing the horizontal type of radius $r$ and weight $t$, or simply $(r, t)$-type. We denote by $\operatorname{TYH}(r, t)$ the set of all possible formulas $\varphi(x)$ constructed for different functions $h$ for fixed $r$ and $t$. For any element $a$ of a model $\mathfrak{M} \in \operatorname{ModFin}(T)$, there is some formula $\varphi(x) \in \operatorname{TYH}(r, t)$ such that $\varphi(a)$ is true. This formula $\varphi(x)$ is called the horizontal type of the element $a$ in the model $\mathfrak{M}$. We write $\tau H(a, r, t)=$ $\tau H(b, r, t)$ if there is a single formula $\varphi(x) \in T Y H(r, t)$ such that both statements $\varphi(a)$ and $\varphi(b)$ are true. Here, $a$ and $b$ may be elements of the same or distinct models of the theory $T$.

A type $\varphi \in \operatorname{TYH}(r, t)$ for $t \in \mathbb{N}$ is called a bounded horizontal type or simply a horizontal type. Such a type $\varphi(x)$ counts the number of points realizing local types in an $H$-class up to the bound $t$ or, if greater than or equal to $t$, it includes the statement that "there exist $\geqslant t$ many such points". Notice that we could also look at total horizontal types which count the exact number of points in the $H$-class realizing each local type $\theta(x) \in T Y(r)$. However, these total types are too categorical for our purposes, so we restrict our attention to bounded horizontal types.

To utilize the Ehrenfeucht back-and-forth method for some fixed number of steps $s$, we need to select the number $t$ sufficiently large (but finite) so that the types TYH $(r, t)$ contain enough information for the back-and-forth method to work. Therefore, we shall consider only bounded horizontal types from now on.

We now study some properties of horizontal types.

Lemma 10.4. For any $r, t \in \mathbb{N}$, the set of types $\operatorname{TYH}(r, t)$ is finite.

Proof. Obvious.

Lemma 10.5. Let $a, b$ be arbitrary elements of a model $\mathfrak{M}$ of the theory $T$. For any $r, t$, if $H(a, b)$ then $\tau H(a, r, t)=\tau H(b, r, t)$. 
Proof. Immediate by definition.

Lemma 10.5 shows that horizontal types actually characterize $H$-classes instead of individual elements. Therefore, when speaking of the number of realizations of a horizontal type, we will always mean the number of $H$-classes on which elements of this type are realized.

A horizontal type of a given radius uniquely determines the existence of the $H$-classes in its neighborhood:

Lemma 10.6. Let $a \in \mathfrak{M}\left\langle\varepsilon^{\prime}\right\rangle$ and $b \in \mathfrak{M}\left\langle\varepsilon^{\prime \prime}\right\rangle$ for $\varepsilon^{\prime}, \varepsilon^{\prime \prime} \in 2^{<\omega}$. Also, let $t>0, i \in \mathbb{Z}$, and $r \in \mathbb{N}$ be such that $|i| \leqslant r$. Then $\tau H(a, r, t)=\tau H(b, r, t)$ implies $(\exists x) D_{i}(a, x) \Leftrightarrow(\exists y) D_{i}(b, y)$.

Proof. Immediate by Lemma 10.1 .

The following simple statement shows that a horizontal type with given parameters comprises the complete description of a unique type with arbitrary smaller parameters.

Lemma 10.7. Let numbers $r^{\prime}, r, t^{\prime}, t \in \mathbb{N}$ be given such that $r^{\prime} \leqslant r$ and $t^{\prime} \leqslant t$. Then for any elements $a \in \mathfrak{M}\left\langle\varepsilon^{\prime}\right\rangle$ and $b \in \mathfrak{M}\left\langle\varepsilon^{\prime \prime}\right\rangle$ with $\varepsilon^{\prime}, \varepsilon^{\prime \prime} \in 2^{<\omega}$, $\tau H(a, r, t)=\tau H(b, r, t)$ implies $\tau H\left(a, r^{\prime}, t^{\prime}\right)=\tau H\left(b, r^{\prime}, t^{\prime}\right)$.

Proof. By direct inspection.

For our purposes, the following two statements are important, namely, that a horizontal type with given parameters uniquely determines a type with smaller parameters not only for this $H$-class, but also for $H$-classes in its neighborhood. We have to distinguish two cases here: how a horizontal type of an element determines the horizontal types of the $H$-classes above it and how it determines those below it.

The following statement is true for all models of the theory $T$ without exception.

Lemma 10.8. Let $k, m \in \mathbb{N}$ be given such that $2 \leqslant k \leqslant s, 0 \leqslant m \leqslant 2 \cdot 3^{k-2}$. Fix arbitrary elements $a, a^{\prime} \in \mathfrak{M}\left\langle\varepsilon^{\prime}\right\rangle$ and $b, b^{\prime} \in \mathfrak{M}\left\langle\varepsilon^{\prime \prime}\right\rangle$ for $\varepsilon^{\prime}, \varepsilon^{\prime \prime} \in 2^{<\omega}$ such that $D_{m}\left(a, a^{\prime}\right)$ and $D_{m}\left(b, b^{\prime}\right)$ holds. Then $\tau H\left(a, 3^{k-1}, s^{*}\right)=\tau H\left(b, 3^{k-1}, s^{*}\right)$ implies $\tau H\left(a^{\prime}, 3^{k-2}, s^{*}\right)=\tau H\left(b^{\prime}, 3^{k-2}, s^{*}\right)$.

Proof. Immediate by Lemma 7.2, given that the type declarations of the elements $a^{\prime}$ and $b^{\prime}$ are completely contained in the type declarations of the elements $a$ and $b$ since the spheres of the elements in the classes $[a]_{H}$ and $[b]_{H}$ completely cover all spheres in the classes $\left[a^{\prime}\right]_{H}$ and $\left[b^{\prime}\right]_{H}$ because of the restricted $D$-distance.

The following statement holds only for the models of the class (9.9), moreover, it applies only with some exceptions.

Lemma 10.9. Let $k, m \in \mathbb{N}$ be given such that $2 \leqslant k \leqslant s, 0<m \leqslant 2 \cdot 3^{k-2}$. 
Fix arbitrary elements $a, a^{\prime} \in \mathfrak{M}\left\langle\varepsilon^{\prime}\right\rangle$ and $b, b^{\prime} \in \mathfrak{M}\left\langle\varepsilon^{\prime \prime}\right\rangle$ for $\varepsilon^{\prime}, \varepsilon^{\prime \prime} \succcurlyeq \varepsilon^{*}\left\lceil w_{0}\right.$ such that $D_{m}\left(a^{\prime}, a\right)$ and $D_{m}\left(b^{\prime}, b\right)$ holds. Then, if $m \notin\left[3^{k-2}+1,2 \cdot 3^{k-2}\right]$; or $m \in$ $\left[3^{k-2}+1,2 \cdot 3^{k-2}\right]$ and one of two following conditions (10.1) or (10.2) holds:

$$
\begin{aligned}
& \left.\left.\left(\forall t \in\left[0.5\left(3^{k-1}+3\right), m+0.5\left(3^{k-2}+1\right)\right]\right)[\urcorner D_{t}(E, a) \&\right\urcorner D_{t}(E, b)\right], \\
& \left(\exists t \in\left[0.5\left(3^{k-1}+3\right), m+0.5\left(3^{k-2}+1\right)\right]\right)\left[D_{t}(E, a) \& D_{t}(E, b)\right],
\end{aligned}
$$

then $\tau H\left(a, 3^{k-1}, s^{*}\right)=\tau H\left(b, 3^{k-1}, s^{*}\right)$ implies $\tau H\left(a^{\prime}, 3^{k-2}, s^{*}\right)=\tau H\left(b^{\prime}, 3^{k-2}, s^{*}\right)$.

PRoOF. As in the previous lemma, the type declarations of $a$ and $b$ contain complete descriptions of the horizontal types of $a^{\prime}$ and $b^{\prime}$, except for the local types in certain small areas near the boundaries $B L$ and $B R$ of the models considered. These areas arise because as we move in the negative $D$-direction, the triangle becomes wider. The spheres, as shown in Fig. 7.1, cannot make up for this change in width in the required number of steps. It follows from our description of these models, see Fig. 9.1 and Lemmas 9.7, 9.8, 9.10 and 9.14 , that the areas near the side boundaries consist mostly of large stretches of continuous blanks. Therefore, in large areas of the models, the types of $a^{\prime}$ and $b^{\prime}$ simply cannot be different. The only exception is the case when the elements $a$ and $b$ are located at certain distances above the point $E$. One can check that for $m \leqslant 3^{k-2}$, any $3^{k-2}$-sphere of an element of $\left[a^{\prime}\right]_{H}$ or $\left[b^{\prime}\right]_{H}$ is completely contained in the $3^{k-1}$-sphere of an element of $[a]_{H}$ or $[b]_{H}$, respectively, so the implication in Lemma 10.9 holds without restrictions. For the case $3^{k-2}<m \leqslant$ $2 \cdot 3^{k-2}$, the conditions (10.1) and (10.2) ensure that either $a$ and $b$ are sufficiently $D$-far away from the point $E$, or that they both have the same $D$-distance to $E$.

We now begin characterizing the local and horizontal types realized in models of the theory $T$. We will consider from now on only local types of classes $T Y\left(3^{k-1}\right)$ and horizontal types of classes $T Y H\left(3^{k-1}, s^{*}\right)$ with $k \leqslant s$, where the parameter $s$ was fixed in (9.1) while the parameter $s^{*}$ was introduced in (9.5). These restrictions will allow us to avoid complicated expressions later.

Now we introduce four important technical definitions.

Definition 1. A local type $\theta(x) \in T Y\left(3^{k-1}\right)$ is called limited in horizontal type $\varphi(x) \in \operatorname{TYH}\left(3^{k-1}, s^{*}\right)$ if there is a number $r<s^{*}$ such that in any finite model $\mathfrak{M}$ of theory $T$, the following formula is true:

$$
(\forall x)\left(\varphi(x) \rightarrow\left(\exists^{r} z\right)[H(x, z) \& \theta(z)]\right) .
$$

Definition 2. A local type $\theta(x) \in T Y\left(3^{k-1}\right)$ is called $s^{*}$-unlimited in horizontal type $\varphi(x) \in T Y H\left(3^{k-1}, s^{*}\right)$ if in any finite model $\mathfrak{M}$ of theory $T$, the following formula is true:

$$
(\forall x)\left(\varphi(x) \rightarrow\left(\exists^{\geqslant} s^{*} z\right)[H(x, z) \& \theta(z)]\right)
$$


Definition 3. Let $w \in \mathbb{N}$ be given. A horizontal type $\varphi(x) \in T Y H\left(3^{k-1}, s^{*}\right)$ is called limited in the class of models (9.4), if there is a number $r<s^{*}$ such that in any model $\mathfrak{M}$ of the class (9.4), the following formula is true:

$$
\left.\left(\exists x_{1} x_{2} \ldots x_{r}\right)\left(\bigwedge_{i \neq j}\right\urcorner H\left(x_{i}, x_{j}\right) \& \bigwedge_{i=1}^{r} \varphi\left(x_{i}\right) \&(\forall x)\left[\varphi(x) \rightarrow \bigvee_{i=1}^{r} H\left(x_{i}, x\right)\right]\right) .
$$

Definition 4. Let $w \in \mathbb{N}$ be given. A horizontal type $\varphi(x) \in T Y H\left(3^{k-1}, s^{*}\right)$ is called $s^{*}$-unlimited in the class of models (9.4), if in any model $\mathfrak{M}$ of the class (9.4), the following formula is true:

$$
\left.\left(\exists x_{1} x_{2} \ldots x_{s^{*}}\right)\left(\bigwedge_{i \neq j}\right\urcorner H\left(x_{i}, x_{j}\right) \& \bigwedge_{i=1}^{s^{*}} \varphi\left(x_{i}\right)\right) .
$$

Note that, by the definitions introduced earlier in this section, for any $k \in$ $\mathbb{N}$, each local type $\theta(x) \in T Y\left(3^{k-1}\right)$ is either limited or $s^{*}$-unlimited in any horizontal type $\varphi(x) \in \operatorname{TYH}\left(3^{k-1}, s^{*}\right)$.

The following properties hold concerning Definitions 3 and 4 .

Lemma 10.10. The following assertions hold:

(a) Let the type $\varphi(x) \in \operatorname{TYH}\left(3^{k-1}, s^{*}\right)$ be limited in the class of models $\mathfrak{M}\left\langle\varepsilon^{\prime}\right\rangle$, $\varepsilon^{\prime} \succcurlyeq \varepsilon^{*} \mid w$. Then the type $\varphi(x)$ is limited in the class of models $\mathfrak{M}\left\langle\varepsilon^{\prime}\right\rangle, \varepsilon^{\prime} \succcurlyeq \varepsilon^{*} \mid$ $w^{\prime}$, for any $w^{\prime}>w$.

(b) Let the type $\varphi(x) \in \operatorname{TYH}\left(3^{k-1}, s^{*}\right)$ be $s^{*}$-unlimited in the class of models $\mathfrak{M}\left\langle\varepsilon^{\prime}\right\rangle, \varepsilon^{\prime} \succcurlyeq \varepsilon^{*} \mid w$. Then the type $\varphi(x)$ is $s^{*}$-unlimited in the class of models $\mathfrak{M}\left\langle\varepsilon^{\prime}\right\rangle, \varepsilon^{\prime} \succcurlyeq \varepsilon^{*} \mid w^{\prime}$, for any $w^{\prime}>w$.

(c) Let $r<k$ and let $\varphi(x) \in \operatorname{TYH}\left(3^{k-1}, s^{*}\right)$ be $s^{*}$-unlimited in the class of models $\mathfrak{M}\left\langle\varepsilon^{\prime}\right\rangle, \varepsilon^{\prime} \succcurlyeq \varepsilon^{*} \mid w$. We denote by $\varphi^{\prime}(x) \in T Y H\left(3^{r-1}, s^{*}\right)$ the natural restriction of $\varphi(x)$ to the smaller radius. Then $\varphi^{\prime}(x)$ is $s^{*}$-unlimited in the same class of models $\mathfrak{M}\left\langle\varepsilon^{\prime}\right\rangle, \varepsilon^{\prime} \succcurlyeq \varepsilon^{*} \mid w$.

Proof. Immediate from the definitions.

Our main purpose now is to establish that in all models $\mathfrak{M}\left\langle\varepsilon^{\prime}\right\rangle$ with $\varepsilon^{\prime} \succcurlyeq$ $\varepsilon^{*} \mid w$ for $w$ sufficiently large, each horizontal type $\varphi(x) \in T Y H\left(3^{s-1}, s^{*}\right)$ is either limited or $s^{*}$-unlimited. With these properties, it will be possible to organize the Ehrenfeucht back-and-forth method on $s$ steps between any two models $\mathfrak{M}\left\langle\varepsilon^{\prime}\right\rangle$ and $\mathfrak{M}\left\langle\varepsilon^{\prime \prime}\right\rangle$ with $\varepsilon^{\prime}, \varepsilon^{\prime \prime} \succcurlyeq \varepsilon^{*} \mid w$.

We consider only models of the class (9.9) from now on. Consider the fol- 
lowing four families of $H$-classes in these models:

$$
\begin{aligned}
& \mathfrak{X}_{1}=\text { the set of } H \text {-classes in the interval from } W \text { to } C T, \\
& \mathfrak{X}_{2}=\text { the set of } H \text {-classes in the interval from }(f) \text { to } W, \\
& \mathfrak{X}_{3}=\text { the set of } H \text {-classes in the interval from }\left(f^{\prime}\right) \text { to }(f) \text {, } \\
& \mathfrak{X}_{4}=\text { the set of } H \text {-classes in the interval from } O \text { to }\left(f^{\prime}\right) .
\end{aligned}
$$

We assume that the boundary $H$-classes between these sets belong to the upper of the two adjacent sets.

Now we consider the following sets of types for models of the class (9.9) and any of its subclasses of the form (9.4) for $w_{0} \leqslant w$ :

$$
\begin{aligned}
& \mathfrak{T}_{k}(\mathfrak{M})=\text { the set of } \operatorname{TYH}\left(3^{s-1}, s^{*}\right) \text {-types realized in zone } \mathfrak{X}_{k} \text { of the model } \mathfrak{M}, \\
& \mathfrak{T}_{k}(w)=\bigcup\left\{\mathfrak{T}_{k}(\mathfrak{M}\langle\varepsilon\rangle)\left|\varepsilon^{*}\right| w \preccurlyeq \varepsilon, \varepsilon \in \operatorname{Hlt}(\mathcal{M})\right\}, \\
& k=1,2,3,4, \\
& \mathfrak{T}(w)=\mathfrak{T}_{1}(w) \cup \mathfrak{T}_{2}(w) \cup \mathfrak{T}_{3}(w) \cup \mathfrak{T}_{4}(w) .
\end{aligned}
$$

First, we establish that these four sets of types are pairwise disjoint for the class of models.

Lemma 10.11. Let $w_{0} \leqslant w^{\prime} \leqslant w^{\prime \prime}$. Then $\mathfrak{T}_{k}\left(w^{\prime}\right) \supseteq \mathfrak{T}_{k}\left(w^{\prime \prime}\right)$ for $k=1,2,3,4$, and $\mathfrak{T}\left(w^{\prime}\right) \supseteq \mathfrak{T}\left(w^{\prime \prime}\right)$.

ProOF. Obvious.

Lemma 10.12. The sets of horizontal types $\mathfrak{T}_{k}\left(w_{0}\right), k=1,2,3,4$, are pairwise disjoint.

Proof. First, to distinguish between the horizontal types in $\mathfrak{T}_{1}\left(w_{0}\right)$ and $\mathfrak{T}_{2}\left(w_{0}\right)$, notice that in the area $\mathfrak{X}_{1}$, the head only moves across blanks, while in the area $\mathfrak{X}_{2}$, the head moves across zeros and ones with an occasional isolated blank. Second, the types in both $\mathfrak{T}_{1}\left(w_{0}\right)$ and $\mathfrak{T}_{2}\left(w_{0}\right)$ differ from the types in $\mathfrak{T}_{3}\left(w_{0}\right)$ and $\mathfrak{T}_{4}\left(w_{0}\right)$ because of the state the machine is in. Finally, to distinguish the types in $\mathfrak{T}_{3}\left(w_{0}\right)$ and $\mathfrak{T}_{4}\left(w_{0}\right)$, notice that every $H$-class in $\mathfrak{T}_{3}\left(w_{0}\right)$ has a point whose local type of radius $3^{s-1}$ contains both a $0^{\prime}$ point and a blank located to the left of the input field (as an example, see Fig. 8.2(b)), while no $H$-class in $\mathfrak{T}_{4}\left(w_{0}\right)$ contains such local types.

We now study properties of horizontal types by separately considering the four classes $\mathfrak{T}_{n}(w)$ for $w \geqslant w_{0}$. Consider types occurring in the final part of the computation.

Lemma 10.13. Let the type $\varphi(x) \in T Y H\left(3^{s-1}, s^{*}\right)$ belong to $\mathfrak{T}_{1}\left(w_{0}\right)$. Then either $\varphi(x)$ is limited in the class of models (9.9), or $\varphi(x)$ is $s^{*}$-unlimited in this class of models. 
Proof. Let $\mathfrak{M}$ be a model of class (9.9). The types of the $H$-classes near the top $C T$, for which the number of realizations of a local type, with its neighborhood of all blanks, does not reach the bound $s^{*}$, are all limited in the class of models (9.9). These are the $H$-classes within $\rho^{*}$-distance $\leqslant 0.5 \cdot\left(s^{*}+s^{+}\right)$ from the top of $\Delta(\mathfrak{M})$. Also, the types of the $H$-classes located within $\rho^{*}$ distance no more than $0.5 s^{+}$from the points $W$ or $E$ are limited in the class of models (9.9). All other types of elements above $W$ are obviously $s^{*}$-unlimited in the class of models (9.9) by the description of these areas in Lemmas 9.8-9.10, together with the inequalities (a) and (b) of Lemma 9.13.

Lemma 10.14. Let the type $\varphi(x) \in T Y H\left(3^{s-1}, s^{*}\right)$ belong to $\mathfrak{T}_{2}\left(w_{0}\right)$. Then either $\varphi(x)$ is limited in the class of models (9.9), or $\varphi(x)$ is $s^{*}$-unlimited in this class of models.

Proof. For this subclass of types, we note that the segment $U W$ has the same length $2\left(l_{0}+l_{1}\right)$ in all models of the class (9.9), and thus, the set of $\left(3^{s-1}, s^{*}\right)$-types generated by the points in this segment, consisting of $\left(l_{0}+l_{1}+1\right)$ horizontal types, is exactly the same in all models of the class (9.9), so these types do not affect the limitedness or $s^{*}$-unlimitedness of any horizontal type in this area. As for the $H$-classes generated by the points in the segment $Z U$, the types within quasidistance $\leqslant 0.5 s^{+}$of $Z$ are all limited in the class of models (9.9), while all the other types are $s^{*}$-unlimited in the class of models (9.9) because of the choice of the parameters $l_{0}$ and $l_{1}$, and by Lemmas 9.7, 9.8, 9.15, 9.16, which describes this area.

Thus, both sets of types $\mathfrak{T}_{1}\left(w_{0}\right)$ and $\mathfrak{T}_{2}\left(w_{0}\right)$ consist only of types limited or $s^{*}$-unlimited in the class of models (9.9).

Among the types intersecting the kernel $S Z C D$, we first consider the types occurring above the line $\left(f^{\prime}\right)$.

Lemma 10.15. Let $\varphi(x) \in \operatorname{TYH}\left(3^{s-1}, s^{*}\right)$ belong to $\mathfrak{T}_{3}\left(w_{0}\right)$. Then either $\varphi(x)$ is limited in the class of models (9.9), or $\varphi(x)$ is $s^{*}$-unlimited in this class of models.

Proof. Remember that we denote the singular point $Z$ by $Z_{0}$ for simplicity (as mentioned above). It can be checked directly that the types of the $H$-classes of each point $Z_{i}, 0 \leqslant i \leqslant s^{+}$, and all of its $k$-th $D$-successors for

$$
k \in \mathbb{Z}, \quad 0<|k| \leqslant \min \left\{3^{s-1}, s^{+}-i\right\},
$$

are limited, since for the corresponding horizontal type from $T Y H\left(3^{s-1}, s^{*}\right)$, one of its local types will cover simultaneously both the blank to the left of the field $I N P U T$ and the singular point $Z_{i}$. In fact, all these horizontal types are realized by a unique $H$-class in any model of the class (9.9).

There are also limited horizontal types in the class $\mathfrak{T}_{3}$ near the singular points $Z_{i}^{\prime}, 1 \leqslant i \leqslant s^{+}$. These types are the types of the points $Z_{i}^{\prime}$ themselves, and 
the $H$-classes of their $k$-th $D$-successors for

$$
k \in \mathbb{Z}, \quad 0<|k| \leqslant i+l_{0},
$$

since for the corresponding type from $\operatorname{TYH}\left(3^{s-1}, s^{*}\right)$, one of its local types will cover simultaneously both the blank to the left of the field INPUT and the symbol $0^{\prime}$ near the left end of the field INPUT, while other local types will cover the singular point $Z_{i}^{\prime}$ or a head position together with the blank line $O P$, or a unique head position inside the rectangle $O P I K$ (that is, a head position together with two or more strips $\varepsilon_{i}$ having values $\left.\varepsilon_{i}=1\right)$. Note that the other $k$-th successors of $Z_{i}^{\prime}$ for $k$ in (10.4) will be $s^{*}$-unlimited horizontal types in models from the class (9.9).

Now we pass to the final part of the proof of Lemma 10.15. All $H$-classes near the singular points $Z_{i}$ and $Z_{i}^{\prime}$, determined by the restrictions (10.3) and (10.4) form a family of horizontal types which is exactly the same in any model of the class (9.9); therefore, these types do not affect the limitedness or $s^{*}$ unlimitedness of any horizontal type in this area on the class (9.9). As for the other types of the region $\mathfrak{X}_{3}$, realized in models of the class $(9.9)$, they are obviously $s^{*}$-unlimited, which immediately follows from Lemma 9.16 together with Lemma 9.5, which establish the general form of a model in this area. Thus, we have that each type in the family $\mathfrak{X}_{3}$ is either limited or $s^{*}$-unlimited in the class of models (9.9).

It remains to consider the most complicated case of the horizontal types intersecting the kernel $S Z C D$ below the line $\left(f^{\prime}\right)$. Because of the simplicity of the structure of the three areas we considered above, we could directly describe all types there and even evaluate the number of realizations of each type. Such an analysis for $\mathfrak{T}_{4}\left(w_{0}\right)$ is impossible because of the arbitrary computations in the kernel of the universal block. Our goal is to show that, nevertheless, even in this area, the types of the $H$-classes for models $\mathfrak{M}=\mathfrak{M}\left\langle\varepsilon^{\prime}\right\rangle$ with $\varepsilon^{\prime} \succcurlyeq \varepsilon^{*} \mid w$ for sufficiently large $w$ are either limited or $s^{*}$-unlimited. Since a direct description is impossible, we must rely on arguments of a more combinatorial character.

Let $\mathfrak{M}$ be one of the models satisfying (9.9). For $k \in \mathbb{N}$, we denote by $\mathfrak{M}[k]$ the $H$-class of $\mathfrak{M}$ which is the $k$-th $D$-successor of the lower boundary $J$ of $\Delta(\mathfrak{M})$ (that is, the $k$-th row of $\mathfrak{M}$ ). $\mathfrak{M}[k]$ is undefined if $k$ exceeds the height of $\mathfrak{M}$. For $k \in \mathbb{N}$, we let $\tau H\left(\mathfrak{M}[k], 3^{s-1}, s^{*}\right)$ denote the horizontal $\left(3^{s-1}, s^{*}\right)$ type of the $H$-class $\mathfrak{M}[k]$ if this class belongs to the area $\mathfrak{X}_{4}$ of $\mathfrak{M}$, i.e., if it is located below $\left(f^{\prime}\right)$ in this model $\mathfrak{M}$. Otherwise, the type $\tau H\left(\mathfrak{M}[k], 3^{s-1}, s^{*}\right)$ is considered undefined.

The following statement is the most important property of the $\left(3^{s-1}, s^{*}\right)$ types realized in the area $\mathfrak{X}_{4}$ of the models of the theory $T$. One could say that this property is the key ingredient in the construction of the theory $T$.

Lemma 10.16. Let $\mathfrak{M}\left\langle\varepsilon^{\prime}\right\rangle$ and $\mathfrak{M}\langle\varepsilon\rangle$ be two models of $T$. Let $\varepsilon \succcurlyeq \varepsilon^{\prime} \succcurlyeq \varepsilon^{*} \mid w_{0}$ 
be such that $\tau H\left(\mathfrak{M}\left\langle\varepsilon^{\prime}\right\rangle[k], 3^{s-1}, s^{*}\right)$ and $\tau H\left(\mathfrak{M}\langle\varepsilon\rangle[k], 3^{s-1}, s^{*}\right)$ are defined. Then we have

$$
\tau H\left(\mathfrak{M}\left\langle\varepsilon^{\prime}\right\rangle[k], 3^{s-1}, s^{*}\right)=\tau H\left(\mathfrak{M}\langle\varepsilon\rangle[k], 3^{s-1}, s^{*}\right)
$$

Proof. Let Fig. 9.1 represent the model $\mathfrak{M}\langle\varepsilon\rangle$. By Lemma 9.17, there is an isomorphic embedding $\mu$ of the kernel $\mathbb{K}\left(\mathfrak{M}\left\langle\varepsilon^{\prime}\right\rangle\right)$ into the kernel $\mathbb{K}(\mathfrak{M}\langle\varepsilon\rangle)$. Let the trapezoid $A B^{\prime} C^{\prime} D^{\prime}$ in Fig. 9.1 represent the part of the image of $\mathbb{K}\left(\mathfrak{M}\left\langle\varepsilon^{\prime}\right\rangle\right)$ under this embedding which lies inside $A B C D$. By our assumption on $k$, both $\mathfrak{M}\left\langle\varepsilon^{\prime}\right\rangle[k]$ and $\mathfrak{M}\langle\varepsilon\rangle[k]$ are located below the line $\left(f^{\prime}\right)$ in the corresponding models. We consider an element $a^{\prime} \in \mathfrak{M}\left\langle\varepsilon^{\prime}\right\rangle$ which represents the class $\mathfrak{M}\left\langle\varepsilon^{\prime}\right\rangle[k]$ in $\mathfrak{M}\left\langle\varepsilon^{\prime}\right\rangle$, and an element $a \in \mathfrak{M}\langle\varepsilon\rangle$ which represents the corresponding class $\mathfrak{M}\langle\varepsilon\rangle\lceil k\rceil$ (see Fig. 9.1).

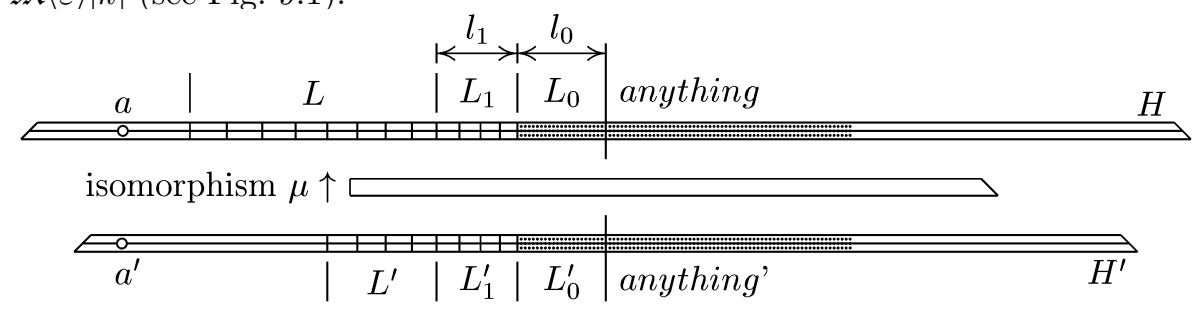

Fig. 10.1. Surroundings of two horizontal classes $\mathfrak{M}\langle\varepsilon\rangle[k]$ and $\mathfrak{M}\left\langle\varepsilon^{\prime}\right\rangle[k]$

In Fig. 10.1, a general picture of the classes $[a]_{H}$ and $\left[a^{\prime}\right]_{H}$ together with their neighborhoods is presented. The clippings of the corresponding areas from the models $\mathfrak{M}\left\langle\varepsilon^{\prime}\right\rangle$ and $\mathfrak{M}\langle\varepsilon\rangle$ as trapezoids of height $s^{+}$are depicted, denoted in the figure by $H$ and $H^{\prime}$. In these trapezoids, all the information necessary for the definition of the horizontal $\left(3^{s-1}, s^{*}\right)$-types of the elements $a$ and $a^{\prime}$ is presented. In the figure, an area is also shown where the isomorphism $\mu$ between $H$ and $H^{\prime}$ acts. Other properties of models in the area of the classes $H^{\prime}$ and $H$ are described in Lemmas 9.3, 9.5-9.8, 9.14.

The areas $L_{0}, L_{1}, L_{0}^{\prime}, L_{1}^{\prime}$ are areas of lengths $l_{0}$ and $l_{1}$, respectively, $L$ and $L^{\prime}$ are the parts of the field INPUT to the left of the areas $L_{1}+L_{0}$ and $L_{1}^{\prime}+L_{0}^{\prime}$, and by "anything", we denote the areas to the right of the central line $\mathrm{OH}$, respectively, in which the current computations are represented. It is necessary to note that the position of the machine head as well as the position $0^{\prime}$ of the pointer $t$ in the field INPUT lie within the domain of the isomorphism $\mu$; therefore, the machine head as well as the pointer $t$ in the area $H$ will be the $\mu$-images of their locations in $H^{\prime}$. Also note that the heights of the areas $H^{\prime}$ and $H$ are equal to $s^{+}$, that is, less than the distances between the adjoining lines $\varepsilon_{i}$ and $\varepsilon_{i-1}$ in the areas $L+L_{1}$ and $L^{\prime}+L_{1}^{\prime}$. Therefore, the line of the head (if it appears within the bounds of $L+L_{1}$ and $L^{\prime}+L_{1}^{\prime}$ ), can lie close to only one of lines $\varepsilon_{i}$ within the bounds of the areas $H$ and $H^{\prime}$.

So, we see that the intervals with arbitrary information $L_{0}^{\prime}+$ anything ${ }^{\prime}$ and $L_{0}+$ anything are isomorphic, the areas $L^{\prime}+L_{1}^{\prime}$ and $L+L_{1}$ are very simple (they 
may include a few special points where $\mu$ acts and more than $s^{*}$ simple isolated strips with information $\varepsilon_{i}=1$ ), while the left and the right end sides of the classes consist of blanks only, which have length more than $s^{*}+s^{+}$. Thus, we have the coincidence of the horizontal types $\tau H\left(a, 3^{s-1}, s^{*}\right)=\tau H\left(a^{\prime}, 3^{s-1}, s^{*}\right)$, and Lemma 10.16 is proved.

Lemma 10.17 For any models $\mathfrak{M}\langle\varepsilon\rangle$ and $\mathfrak{M}\left\langle\varepsilon^{\prime}\right\rangle$ with $\varepsilon^{\prime} \succcurlyeq \varepsilon \succcurlyeq \varepsilon^{*}\left\lceil w_{0}\right.$, we have $\mathfrak{T}_{4}(\mathfrak{M}\langle\varepsilon\rangle) \subseteq \mathfrak{T}_{4}\left(\mathfrak{M}\left\langle\varepsilon^{\prime}\right\rangle\right)$.

ProOF. This lemma follows directly from Lemma 10.16 since the vertical distance from $J$ up to $\left(f^{\prime}\right)$ in $\mathfrak{M}\langle\varepsilon\rangle$ increases as $|\varepsilon|$ increases.

Now, we turn to our main combinatorial statement:

Lemma 10.18. There is a number $w^{*} \geqslant w_{0}$ such that any type $\varphi(x) \in \mathfrak{T}_{4}\left(w^{*}\right)$ is either limited in the class of models

$$
\mathfrak{M}\langle\varepsilon\rangle, \quad \varepsilon^{*} \uparrow w^{*} \preccurlyeq \varepsilon, \quad \varepsilon \in \operatorname{Hlt}(\mathcal{M}),
$$

or $\varphi(x)$ is $s^{*}$-unlimited in this class of models.

PROOF. The proof of this statement is based on the special form of the program of the universal block presented in Fig. 5.5. In some cases, we consider the universal block without any references to its internal structure, in other cases, its internal structure is considered. Though the program of the universal block really uses only the parameters $n$ and $e$ (see Section 5), we will make references to it with three parameters of the form

$$
U(n, x, e),
$$

that will allow us not only to refer to the parameters used by it, but also to show the condition of the tape at the stage of the call of the block. There is the relation $e=\operatorname{Nom}\left\langle\varepsilon_{0}, \varepsilon_{1}, \ldots, \varepsilon_{x-1}\right\rangle$ among the parameters $x$ and $e$ at the stage when the universal block is called, where the values $\varepsilon_{i}$ are taken from the bit field INPUT.

Recall that we restrict ourselves only to models of the class (9.9).

According to the algorithm $\mathcal{M}$, at each entry into the universal block and then at the exit from it, the head of the machine passes the field of the cell $n$ by multiple executions of one of two following commands

$$
1 q_{75} \rightarrow 1 q_{75} L, \quad 1 q_{6} \rightarrow 1 q_{6} R
$$

The corresponding movements of the head can be seen in Fig. 8.1. When $n$ is small, the description of a horizontal $\left(3^{s-1}, s^{*}\right)$-type can encompass an area of the universal block and simultaneously some fragments of surrounding quasiperiodic computations, and it can even cover areas of two or more universal blocks. In that case, we say that the horizontal types of the call of the universal 
block are not separated from the types of surrounding quasi-periodic computations. But, if $n$ exceeds $s^{+}$, then $\left(3^{s-1}, s^{*}\right)$-types of $H$-classes intersecting the work area of the universal block do not depend on the fragments of the surrounding quasi-periodic computations since they are isolated from those by long enough lines of head movements by commands (10.6). In this latter case, we say that the horizontal types of the call of the universal block are separated from the types of the surrounding quasi-periodic computations. Also, it is necessary to note that the polynomial subroutine in the universal block is separated similarly from the farther part of the program by the head movement over the cell $K E Y$, when its value is large enough.

Some concept of separability can be defined also for the kernel of the universal block in relation to the quasi-periodic computations inside the universal block. One of the ideas of the flow-chart shown in Fig. 5.5 is that the working cell STEP separates the kernel from the surrounding computations. In Fig. 5.5, after erasing $K$, the algorithm adds one to STEP and then the head must move across the string of 1 bits representing STEP before entering the kernel of the universal block. Therefore, all $H$-classes located at a quasidistance $>3^{s-1}$ from the areas of calls to the kernel in the universal block are called the surrounding quasi-periodic computations. In the internal cycle for $S T E P=0,1,2, \ldots$, while $S T E P<s^{+}$, the type of the kernel in the universal block is not separated from the types of the surrounding quasi-periodic computations, while when $S T E P \geqslant s^{+}$, these types are separated from each other. Thus, at each call of the universal block, the computations of its kernel at first are not separated from the surroundings, while after fixed number of stages of the internal cycle with cell $S T E P$, they become separated from the types of the surrounding quasi-periodic computations.

Let $U(n, x, e)$ be a call of the universal block in a model $\mathfrak{M}$ of the class (9.9). By $\mathfrak{T} U(n, x, e)$, we denote the set of horizontal $\left(3^{s-1}, s^{*}\right)$-types realized in the area $U(n, x, e)$ of model $\mathfrak{M}$. It is assumed that all horizontal types of $H$-classes of the computation $U(n, x, e)$, and also of all $H$-classes at a quasidistance $\leqslant 3^{s-1}$ from it, are included in $\mathfrak{T} U(n, x, e)$. The types of $H$-classes at a quasidistance > $3^{s-1}$ from the areas of calls of the universal block are included in the surrounding quasi-periodic computations.

Now, we prove a general fact about calls of the universal block.

Claim A. Let $U(n, x, e)$ be a call of the universal block in a model $\mathfrak{M}$ of the class (9.9) such that $n \geqslant s^{+}$, i.e., the computation $U(n, x, e)$ is separated from the surrounding quasi-periodic computations. Then the set $\mathfrak{T} U(n, x, e)$ is completely defined by the values of its input parameters $n, x$, and $e$.

Proof. By our construction, a call of the universal block starts at stage (d) and the computation exits the block at stage (e) shown in Fig. 5.3. By (5.12), the computation does not shift its head farther than the left end of cell $n$, so 
it is completely located in the trapezoid $O P C D$ shown in Fig. 9.1. Moreover, by Lemma 9.14 both blank areas $S Z X L$ and $D C Y R$ on either side are large enough in models of the class (9.9). Therefore, the set of types $\mathfrak{T} U(n, x, e)$ will be the same in all models of the class (9.9) provided that the computation is separated from the surrounding quasi-periodic computations, which holds under our assumption $n \geqslant s^{+}$.

From now on, we use the function $q(x)$ as defined in (5.6). The next technical statement is important for us:

Claim B. There are integer parameters $t_{0}>s^{+}$and $n_{0}>4 s^{+}$satisfying the following properties:

(a) $\left(\forall s<t_{0}\right)\left[q(s)<n_{0}\right]$,

(b) $\left(\forall s \geqslant t_{0}\right)\left[q(s)>4 s^{+}\right]$,

(c) for each call of the universal block $U(n, x, e)$ with $n \geqslant n_{0}$ in a model of the class (9.9), its internal cycle by cell STEP takes $>t_{0}$ stages.

Proof. First, we can choose $t_{0}>s^{+}$satisfying (b) by the requirement (5.8). Then we choose $n_{0}=\max \left\{4 s^{+}+1, t_{0}+1, q(0), q(1), \ldots, q\left(t_{0}-1\right)\right\}$. As a result, we have (a), while (c) holds by Lemma 5.4 .

The parameters $n_{0}$ and $t_{0}$ chosen in Claim $\mathrm{B}$ will be used again in this section. The fact that $n_{0}>t_{0}$ will be used frequently without being explicitly stated.

Now, we describe the regions where special computations are separated (in some strong sense) from the quasi-periodic computations.

Claim C. Let, in a model of the class (9.9), a call of the universal block be given of the form $U(n, x, e)$ with $n \geqslant n_{0}$. Then

(a) the computation $U(n, x, e)$ is separated from the surrounding quasi-periodic computations outside the universal block,

(b) the polynomial computation in $U(n, x, e)$ is separated from the further part of the computation inside the universal block,

(c) the internal cycle with cell STEP makes $\geqslant t_{0}$ steps in the computation,

(d) after the stage of the computation when the cell STEP reaches the value of $t_{0}$, the calls of the kernel computation are separated from the surrounding quasi-periodic computations inside the universal block.

Proof. By Lemma 5.4 and inequalities (2.6) and (5.7), together with the properties of the parameters $n_{0}$ and $t_{0}$ stated in Claim B.

Now our main goal is to prove some properties of sets of types $\mathfrak{T} U(n, x, e)$ which are stronger than Claim A. Namely, we are going to prove that $\mathfrak{T} U(n, x, e)$ is uniquely determined just by $x$ for small $x$ and large enough $n$, and that this set is independent of the values of the parameters $n, x$ and $e$ when both $n$ and 
$x$ are large enough.

To this end, we study sets of types generated by a call of the universal block.

Let a call $U(n, x, e)$ of the universal block be given in a model $\mathfrak{M}$ of the class (9.9). Let $S T E P_{\downarrow}$ denote the length of the internal cycle by cell $S T E P$, i.e., when $S T E P=S T E P_{\downarrow}$, the exit out of the universal block occurs. Consider the following sets of horizontal $\left(3^{s-1}, s^{*}\right)$-types generated separately by special computations and quasi-periodic computations during the internal cycle (see Fig. 5.5):

$$
\begin{aligned}
& \mathfrak{T} U(n, x, e)^{p o l} \text { is the set of types in the interval from the entry into } \\
& \text { the universal block up to stage }(\mathrm{h}) \text { of the block, } \\
& \mathfrak{T} U(n, x, e)\left[0, t_{0}\right] \text { is the set of types in the interval from the stage }(\mathrm{h}) \\
& \text { of the universal block up to stage }(\mathrm{j}) \text { with } S T E P=t_{0},
\end{aligned}
$$

From now on in this section, by "types" we mean horizontal $\left(3^{s-1}, s^{*}\right)$-types. It must be noted that (except for Claim K below) in (10.7)-(10.11), we consider only the existence of the realized types, not the number of realizations of each type. This makes the remaining proofs in this section simpler.

Note that stage (h) itself is included in the set of types (10.7), and simultaneously, it is excluded from the set of types (10.8). As usual, we include in (10.9) the types of $H$-classes of the kernel computation itself as well as the types of the $H$-classes at a quasidistance $\leqslant 3^{s-1}$ of it. At the same time, we exclude from the sets of types (10.8), (10.10) and (10.11) those located at a quasidistance $\leqslant 3^{s-1}$ from the kernel computation.

The following gives a decomposition of the set of types generated by a call of the universal block into five parts:

$$
\begin{aligned}
\mathfrak{T} U(n, x, e)= & \mathfrak{T} U(n, x, e)^{p o l} \cup \mathfrak{T} U(n, x, e)\left[0, t_{0}\right] \\
& \cup \bigcup_{t_{0} \leqslant S T E P \leqslant S T E P_{\downarrow}} \mathfrak{T} U(n, x, e)^{\text {ker }}[S T E P] \\
& \cup \bigcup_{t_{0} \leqslant S T E P<S T E P_{\downarrow}} \mathfrak{T} U(n, x, e)^{q u a}[S T E P] \\
& \cup \mathfrak{T} U(n, x, e)^{q u a}\left[S T E P_{\downarrow}\right] .
\end{aligned}
$$

Note that this formula is applicable when $n \geqslant n_{0}$, where, by Claim C, the polynomial part is separated from the subsequent computation, and the call of the 
universal block is separated from the surrounding quasi-periodic computations after reaching $S T E P=t_{0}$; moreover, the range of the local cycle exceeds $t_{0}$, which makes all parts of the formula (10.12) defined.

Note $\left[4 s^{+}\right]$. From now on, we often use a parameter of the form $4 s^{+}$, which has the following meaning. Quasi-periodic operations over cells in the program of the universal block may use one or two working pointers $1^{\prime}$. Namely, addition operations may obviously be programmed with just one pointer $1^{\prime}$ in each cell, while the computation of $c(n, e)$ by the rule $\frac{1}{2}(n+e)(n+e+1)+n$ may use a temporary cell for $n+e$ and two pointers $1^{\prime}$ in the cell simultaneously. To compute the square of $c(n, e)$, we can simply duplicate the cell and then use just one pointer $1^{\prime}$ in each of these two cells. So, it is possible to have a situation when two $1^{\prime}$ and the head are on a cell at a stage in the computation. Then, if the value of the cell is less than $4 s^{+}$, a description of a horizontal type may include complete information about the exact value of the cell. Conversely, if the value of a cell is larger than $4 s^{+}$, independently of its real value, the description of a horizontal type will be the same because it cannot encompass the four parts of the cell as a whole. The same argument applies to the comparison operation over cells $K E Y$ and $K$. Different small values of the difference $d$ between the values of $K E Y$ and $K$ may generate different types, while in both cases $K E Y<K-4 s^{+}$ and $K E Y-4 s^{+}>K>4 s^{+}$, the comparison operation generates the same set of types in either of these two cases. So we can say that the value $4 s^{+}$plays the role of a real bound between small values of cells generating isolated types, and large values of cells generating a type independent of the exact content of a cell involved in the computation.

Consider the following sequence of cases for the value of the parameter $x$, and relations between working cells $K E Y$ and $K$ at an instance STEP of the internal cycle:

$$
\begin{aligned}
& \left(\mathrm{X}_{k}\right): x=k, k=0,1,2, \ldots, 4 s^{+}, \quad\left(\mathrm{X}_{\infty}\right): 4 s^{+}<x \leqslant n, \\
& (\mathrm{~L}): K E Y<K, \quad(\mathrm{E}): K E Y=K, \quad(\mathrm{G}): K E Y>K>4 s^{+} .
\end{aligned}
$$

We will use them in the analysis of the sets of realized types.

Now, we study some properties of sets of types presented in formula (10.12).

Claim D. Let $n \geqslant n_{0}$ be given. Then the set $\mathfrak{T} U(n, x, e)^{\text {pol }}$ is completely determined for each of the $4 s^{+}+2$ cases of the value of cell $x$ given in (10.13).

Proof. This statement is actually an important (though obvious) property of quasi-periodic computations, when a subroutine computes the value of a rational polynomial, having positive integer values for positive integer values of arguments. Moreover, by (2.6), the value of the polynomial is large for large values of its parameters. As for the call $U(n, x, e)$ with $n \geqslant n_{0}$, in the case when $x$ is fixed such that $0 \leqslant x \leqslant 4 s^{+}$, the set of types may depend on the parameter 
$e$ which can have small value. But in this case, by Lemma 9.19, the value of $e$ is uniquely defined by $x$ for the call $U(n, x, e)$ in a model of the class (9.9).

The following property is provided by using of $c^{2}(n, e)$ in the universal block:

Claim E. Let a call of the universal block $U(n, x, e)$ be given in a model of the class (9.9) such that $n \geqslant n_{0}$. Consider the value of the cell KEY in this call, and the value of the cell $K$ at a stage of the internal cycle with cell STEP. Then we have

(a) $K E Y>K+4 s^{+}$, if $K E Y>K$,

(b) $K E Y<K-4 s^{+}$, if $K E Y<K$,

(c) $K>4 s^{+}$, if $S T E P \geqslant t_{0}$.

Proof. Since $n \geqslant n_{0}>4 s^{+}$by virtue of Claim B, we have $K E Y=k^{2}$ with $k>4 s^{+}$by inequality (2.6) as well as by flow-chart shown in Fig 5.5. On the other hand, the value of $K$ may be of the form either $k_{1}^{2}$ or $k_{1}\left(k_{1}+1\right)$ for some $k_{1}$ by the definition of function $q(x)$. This immediately gives the properties (a) and (b). Part (c) is a consequence of Claim B (b).

Claim F. Let $n \geqslant n_{0}$ be given. Then the set $\mathfrak{T} U(n, x, e)\left[0, t_{0}\right]$ is completely determined for each of the $4 s^{+}+2$ cases of the value of cell $x$ given in (10.13).

Proof. Directly from the flow-chart given in Fig 5.5 and by Claim B establishing the conditions of the parameters $n_{0}$ and $t_{0}$. By part (a) of Claim B together with the inequality (2.6), we have $K E Y>K$ at all stages of the internal cycle from 0 to $t_{0}$. By Claim $\mathrm{E}$ we have $K E Y>K+4 s^{+}$at these steps. Therefore, this part of the whole computation is independent of $n$ and completely determined by the input parameters $x$ and $e$ which have an effect on the types of this part of a computation.

If one of the conditions $X_{k}, 0 \leqslant k \leqslant 4 s^{+}$is true, by Lemma 9.19, the value of $e$ is uniquely defined by $x$, so the set $\mathfrak{T} U(n, x, e)\left[0, t_{0}\right]$ is completely determined for this $k$. If $X_{\infty}$ is true, the value of $e$ becomes large enough by (2.3). So, this part of the computation will generate the same set of types as well.

Claim G. Let $n \geqslant n_{0}$ be given. Then the set $\mathfrak{T} U(n, x, e)^{k e r}[S T E P]$ with $t_{0} \leqslant S T E P \leqslant S T E P_{\downarrow}$ is completely determined by the value of the cell STEP, and it is independent of any other parameters.

Proof. Directly from the flow-chart given in Fig 5.5 taking into account that the requirement (5.13) on the kernel computation does not allow the program to move its head further than the left end of the cell STEP (which in fact is the only input parameter of the subroutine). Remember that, by Claim C, the kernel computation here is separated form the surrounding quasi-periodic computations inside the universal block.

Note. The statements of Claim G together with Lemma 5.4 represent the so-called quasi-monotonicity property of the kernel computations inside the 
universal block. It is directly provided by the algorithm in Fig. 5.5.

Claim H. Let $n \geqslant n_{0}$ be given. Then the set $\mathfrak{T} U(n, x, e)^{q u a}[S T E P]$ with $t_{0} \leqslant S T E P<S T E P_{\downarrow}$ is completely determined by which of the $4 s^{+}+2$ cases for the value of cell $x$ given in (10.13) holds, and which of the cases $(\mathrm{L})$ or $(\mathrm{G})$ of (10.14) holds at the instance STEP.

Proof. Immediately from the flow-chart given in Fig 5.5 and the properties of the parameters $t_{0}$ and $n_{0}$. Suppose that condition $(\mathrm{G})$ is true. By Claim E we have $K E Y-4 s^{+}>K>4 s^{+}$at the stage, therefore, this part of the whole computation is completely determined by the input parameters $x$ and $e$, whose values can influence on the types. If one of the conditions $\left(\mathrm{X}_{k}\right), 0 \leqslant k \leqslant 4 s^{+}$ is true, by Lemma 9.19, the value of $e$ is uniquely defined by $x$, so the set $\mathfrak{T} U(n, x, e)^{q u a}[S T E P]$ is completely determined for this $k$. If $\left(\mathrm{X}_{\infty}\right)$ is true, the value of $e$ becomes large enough by (2.3). So, this part of the computation will define the same set of types as well. Similar arguments can be applied to the case when $(\mathrm{L})$ is true.

Claim I. For any $x \in \mathbb{N}$, there are infinitely many integers $n>n_{0}$ such that in the call of the universal block of the form

$$
U(n, x, e), \quad e=\operatorname{Nom}\left\langle\varepsilon_{0}, \varepsilon_{1}, \ldots, \varepsilon_{x-1}\right\rangle,
$$

where bit cells $\varepsilon_{i}$ are taken from the sequence (9.2), each of cases $(\mathrm{L}),(\mathrm{E})$ and $(\mathrm{G})$ of (10.14) occurs at least one time in the computation $U(n, x, e)$ (at different stages of its internal cycle).

Proof. Since the bit cells $\varepsilon_{i}$ are taken from the sequence (9.2), and $\varepsilon^{*} \in$ Cons ${ }^{*}$, by (4.19), we have that $\left\langle\varepsilon_{0}, \varepsilon_{1}, \ldots, \varepsilon_{x-1}\right\rangle \in$ Cons. Then, by Lemma 5.2, the relation $U^{\prime}(n, e)$ is true for all $n \in \mathbb{N}$. By the construction of the function $q(x)$ described in Section 5 , for every $n \geqslant x$, a sequence of values

$$
\ldots, c^{-}(n, e), \ldots, c^{+}(n, e), \ldots, c^{2}(n, e),
$$

will be inserted as a row of values of the function $q(x)$. Moreover, a sequence (10.15) for given $e$ will be inserted for different values of $n$ infinitely many times as some values of function $q(x)$. For given $n \geqslant x$ and $e$, consider the least integer $k=k(n, e)$ such that $q(k)=c^{2}(n, e)$ (it is these minimal values which cause the exit out of the universal block). Then, obviously, for any fixed $e$, we have $k(n, e) \rightarrow \infty$ for $n \rightarrow \infty$. This immediately gives the statement of Claim I. Note an obvious fact, namely, that case (E) occurs at the last stage $S T E P_{\downarrow}$ of the internal cycle in the computation.

Claim J. Let $n \geqslant n_{0}$ be given. Then the set $\mathfrak{T} U(n, x, e)^{q u a}\left[S T E P_{\downarrow}\right]$ is completely determined for each of the $4 s^{+}+2$ cases of the value of the cell $x$ given in (10.13).

Proof. Directly from the flow-chart given in Fig 5.5, showing that the comparison operation (ending with the "yes" branch) as well as the further exit 
operations generate the same set of types because the input parameter $n$ is large, while $x$, and $e$ are either small and fixed, in case $\left(\mathrm{X}_{k}\right)$, or both of them are large enough, in case $\left(\mathrm{X}_{\infty}\right)$.

Now we prove the following four important technical statements.

The first one is:

Claim K. Let $s^{\prime}$ be the stage in a computation in a model $\mathfrak{M}$ of the class (9.9) when the cell $n$ reaches the value $n_{0}$. Then the family of $\left(3^{s-1}, s^{*}\right)$-types realized in $H$-classes at a quasidistance $\leqslant s^{\prime}$ from the starting class $J$ (counting the number of their realizations) is the same independently of what model $\mathfrak{M}$ was taken.

Proof. Immediate by Lemma 10.16, taking into account that the computations in the interval $\left[0, s^{\prime}\right]$ in all models of the class (9.9) are obviously the same because they use the same initial segment of information from $\varepsilon^{*}$, which in fact is included in $\varepsilon^{*} \uparrow w_{0}$.

The second one:

Claim L0. There is an integer value $w>w_{0}$ such that any $\left(3^{s-1}, s^{*}\right)$-type of quasi-periodic computations outside of the universal block in the zone $\mathfrak{X}_{4}$ of a model of the class (9.9), which is realized after the stage when cell $n$ reaches value $n_{0}$, has $\geqslant s^{*}$ realizations in the model $\mathfrak{M}\left\langle\varepsilon^{*} \mid w\right\rangle$.

Proof. Consider a stage $s^{\prime}$ in the computation with $\operatorname{INPUT}\left\langle\varepsilon^{*} \mid w\right\rangle$ with large enough $w$, when the working cell $n$ reaches the value $n_{0}$. By virtue of Claim $\mathrm{C}$, the types of quasi-periodic computations outside of the universal block after the stage $s^{\prime}$ are separated from types generated by calls of the universal block. It is obvious that the types of quasi-periodic fragments of computations in the region after the stage $s^{\prime}$ depend on the contents of the field INPUT as a whole to some degree. In particular, they do not always depend on the order of the bit values. In the area $O P I K$, where the bit values are close to each other, the types of quasi-periodic computations may depend on the values of the cells $\varepsilon_{i}$. But this dependence is the same for all models of the class (9.9) by virtue of Lemma 9.2. As for the saturation of types in the quasi-periodic computations in the part of the field INPUT located to the left of the line $I K$, it is only important that in the input bit sequence $\varepsilon^{*} \uparrow w$ there are a lot of zero cells $\varepsilon_{i}$ and lot of one cells $\varepsilon_{j}$. Their relative position is irrelevant. The necessary number of zeros and ones in $\varepsilon^{*} \mid w$ can be obtained for large enough value of $w>w_{0}$, since by Lemma 4.1, in a sequence (9.2) there are infinitely many zeros and infinitely many ones. Therefore, the requirement of Claim L0 will be true for a suitably large value of $w$.

The third one:

Claim L1. Let $x$ be fixed such that $0 \leqslant x \leqslant 4 s^{+}$. Then there is a number $n^{\prime}=n^{\prime}(x)$ depending on $x$ such that the set $\mathfrak{T} U(n, x, e)$ is the same for all calls 
$U(n, x, e)$ with $n \geqslant n^{\prime}$ in all models $\mathfrak{M}$ of the class (9.9)

Proof. The set $\mathfrak{T} U(n, x, e)$ consists of five parts as presented in (10.12). Both the first and the second part depend just on the value of $x$ by Claims D and F. By Claim G, the third part is monotonically increasing as the cell STEP increases, and consequently it must stabilize by Lemma 5.4, since the set of horizontal types is finite. Further, Claim $\mathrm{H}$ shows the fourth set depends only on $x$, while Claim I ensures the saturation of the set with various types and therefore its stabilization. The fifth set depends on the value of $x$ by Claim J. As a result, we have the necessary statement for the given $x$ and large enough value of $n^{\prime}$.

The fourth one:

Claim L2. There is an integer $n^{\prime \prime}>0$ such that for any two calls of the universal block $U\left(n_{1}, x_{1}, e_{1}\right)$ and $U\left(n_{2}, x_{2}, e_{2}\right)$ with $n_{1}, n_{2} \geqslant n^{\prime \prime}$ and $x_{1}, x_{2}>4 s^{+}$ and in any two models of the class (9.9), $\mathfrak{T} U\left(n_{1}, x_{1}, e_{1}\right)=\mathfrak{T} U\left(n_{2}, x_{2}, e_{2}\right)$.

Proof. The set $\mathfrak{T} U(n, x, e)$ consists of five parts according to (10.12). The first and second parts are the same for all calls of this form by virtue of Claims D and F. By Claim G, the third part is monotonically increasing as the cell STEP increases, and consequently it must stabilize by virtue of Lemma 5.4, since the set of horizontal types is finite. Further, Claim H states the uniqueness of the fourth part for cases (L) and (G) of (10.14), while Claim I ensures the saturation of the set with various types, and therefore its stabilization. The fifth set is the same for all calls of this form by Claim J. As a result, we obtain the necessary statement for a large enough value of $n^{\prime \prime}$.

Now we turn directly to the proof of Lemma 10.18.

The general technique of the proof is to give an analysis of all calls of the universal block and the surrounding quasi-periodic operations in a computation in a model of the class (9.9), and on this basis we describe the set of realized types, which will imply Lemma 10.18.

We consider a computation of the algorithm $\mathcal{M}$ in a fixed model of the theory $T$ of the form

$$
\mathfrak{M}\left\langle\varepsilon^{*} \mid w\right\rangle, \quad w>w_{0} .
$$

Note that the finite model (10.16) exists for all $w$ by virtue of properties (4.17), (4.18) and (9.2), and for $w>w_{0}$ it belongs to the class (9.9).

Denote by $\varphi[k](x)$ the following horizontal type in $\mathfrak{X}_{4}$ :

$$
\begin{aligned}
& \varphi[k](x)=\mathfrak{M}\left\langle\varepsilon^{*} \mid v\right\rangle[k], \\
& \text { for } v>w_{0} \text { such that } \mathfrak{M}\left\langle\varepsilon^{*} \mid v\right\rangle[k] \text { is defined, } k \in \mathbb{N} .
\end{aligned}
$$

Since the vertical distance from $J$ up to $\left(f^{\prime}\right)$ in $\mathfrak{M}\langle\varepsilon\rangle$ increases as $|\varepsilon|$ increases, the type (10.17) is defined for all $k \geqslant 0$. By Lemma 10.16, the type (10.17) 
is defined uniquely, independently of the choice of the temporary parameter $v$. Obviously, any finite segment of the infinite sequence (10.17) can be realized in a finite model of the form (10.16) for large enough $w>w_{0}$. Furthermore, we assume that $w$ in (10.16) is chosen large enough so that all situations considered below occur in the computation in the area $\mathfrak{X}_{4}$ of the model $\mathfrak{M}$.

We now pass to the analysis of the types realized in $\mathfrak{X}_{4}$.

Consider a stage $s_{0}$ in the computation (10.16) (that is, the $H$-class of this model) when the working cell $n$ reaches the value $n_{0}$. By Claim $\mathrm{C}$, after stage $s_{0}$, the types of the quasi-periodic computations are separated from the types generated by calls of the universal block, and the types of the polynomial part are separated from the subsequent computation in the universal block. The types realized in the interval $\left[0, s_{0}\right]$, by Lemma 10.16 , are the same for all models of the class (9.9), and therefore for all models of its subclass (10.5), they do not present an obstacle to Lemma 10.18; we will therefore generally drop them from our considerations.

Find a value of $s_{1}>s_{0}$ such that in the computation in the interval $\left[s_{0}, s_{1}\right]$ of the model (10.16), every type of a fragment of a quasi-periodic computation which is available in some model of the class (9.9) after the stage $s_{0}$ actually occurs in the model (10.16), and moreover, each of these types has $\geqslant s^{*}$ realizations. Such a value $s_{1}$ exists by Claim L0.

Consider the parameter

$$
n^{*}=\max \left\{n^{\prime}(0), n^{\prime}(1), \ldots, n^{\prime}\left(4 s^{+}\right), n^{\prime \prime}\right\},
$$

where $n^{\prime}(x)$ and $n^{\prime \prime}$ are defined in Claim L1 and Claim L2. Then it is obvious that both Claim L1 and Claim L2 are still valid if we take $n^{\prime}(i)=n^{*}$ for $i=$ $0,1, \ldots, 4 s^{+}$, and $n^{\prime \prime}=n^{*}$.

Now, we turn to types generated by areas of calls of the universal block. As described earlier, we have to consider only the calls $U(n, x, e)$ in the area after the stage $s_{0}$, where universal computations are separated from the surrounding quasi-periodic computations. Divide all such calls of the universal block in the following classes by the values of their input parameters:

$$
\begin{aligned}
& \text { (A) : } U(n, x, e), n<n^{*}, x \in \mathbb{N}, \\
& \left(\mathrm{B}_{x}\right): U(n, x, e), n \geqslant n^{*}, x=0,1, \ldots, 4 s^{+}, e=\operatorname{Nom}\left\langle\varepsilon_{0}, \varepsilon_{1}, \ldots, \varepsilon_{x-1}\right\rangle, \\
& \text { (C) : } U(n, x, e), n \geqslant n^{*}, x>4 s^{+} .
\end{aligned}
$$

There are at most $\left(n^{*}+1\right)^{2}$ calls of the form (A) in any model, because the inequality $x \leqslant n$ is true for each call of the universal block in the computation of the algorithm $\mathcal{M}$, and there can be only one call $U(n, x, e)$ with given $n$ and $x$ in the entire computation. Consider a number $s_{2}>s_{0}$, for which 
- all calls of the universal block of the form (A) occur before stage $s_{2}$ in the model (10.16).

Such a number $s_{2}$ exists by Lemma 8.1.

Now we pass to the calls of the form $\left(\mathrm{B}_{x}\right)$ and $(\mathrm{C})$. By Claim L1, the set of types $\mathfrak{T} U(n, x, e)$ is the same for all calls of the universal block of the form $\left(\mathrm{B}_{x}\right)$ for each value $x=0,1, \ldots, 4 s^{+}$, and by Claim L2, the set of types $\mathfrak{T} U(n, x, e)$ is the same for all calls of the universal block of the form (C).

Consider a number $s_{3}>s_{2}$ for which the following conditions hold:

- for every $x=0,1, \ldots, 4 s^{+}$, there are more than $s^{*}$ calls of the universal block of the form $\left(\mathrm{B}_{x}\right)$ before the stage $s_{3}$ in the model (10.16).

- there are more than $s^{*}$ calls of the universal block of the form $(\mathrm{C})$ before the stage $s_{3}$ in the model (10.16).

One can see that such a number $s_{3}$ exists by Lemma 8.1.

At last, we consider the value $s_{4}=\max \left\{s_{1}, s_{3}\right\}$.

Then we take a number $w^{*}$ such that in the models of the class (10.5), the computations in the area $\left[0, s_{4}\right]$ do not use the information of the field INPUT except during the initial segment $\varepsilon^{*} \uparrow w^{*}$ of the sequence (9.2). That is, the work of the initial $s_{4}$ stages in all models of the class (10.5) must proceed in exactly the same manner. As for the computations after stage $s_{4}$, the work of the algorithm $\mathcal{M}$ can be different in different models of the class (10.5) since the computation may be defined by the input information $\varepsilon_{i}$ taken outside of the limits of the segment $\varepsilon^{*} \mid w^{*}$.

By the choice of the parameter $w^{*}$ and the auxiliary parameters $s_{0}, s_{1}, s_{2}, s_{3}$ and $s_{4}$, we obtain that in the minimal model $\mathfrak{M}\left\langle\varepsilon^{*} \mid w^{*}\right\rangle$ of the class (10.5), all special types in the area $\left[0, s_{0}\right]$, as well as the types of all special calls of the form (A), are realized. At the same time, in the model $\mathfrak{M}\left\langle\varepsilon^{*} \mid w^{*}\right\rangle$, all types of quasi-periodic computations available in models of the class (9.9) are realized $\geqslant s^{*}$ many times, as are all types of calls of the universal block of the form $\left(\mathrm{B}_{x}\right)$ for $x=0,1, \ldots, 4 s^{+}$, as well as all types of calls of the form (C). Hence, our value of $w^{*}$ satisfies the claim of our lemma.

Thus, the statement of Lemma 10.18 is completely proved.

This lemma completes the purely technical part of the combinatorics of our construction. We now apply these results in order to prove our final statement that condition (4.25) holds. 


\section{The Ehrenfeucht property}

Now we consider the class of models of the theory $T$ of the following form

$$
\mathfrak{M}\left\langle\varepsilon^{\prime}\right\rangle, \varepsilon^{\prime} \succcurlyeq \varepsilon^{*} \mid w^{*}
$$

where the bound $w^{*}$ is defined as in Lemma 10.18 .

The following lemma summarizes the results of the last section about the types occurring in models of the theory $T$ from the class (11.1).

Lemma 11.1. The following assertions hold:

(a) Let $a=E\left(\mathfrak{M}\left\langle\varepsilon^{\prime}\right\rangle\right)$ (i.e., the point $E$ in the model $\left.\mathfrak{M}\left\langle\varepsilon^{\prime}\right\rangle\right)$ and $b=E\left(\mathfrak{M}\left\langle\varepsilon^{\prime \prime}\right\rangle\right)$ (i.e., the point $E$ in the model $\left.\mathfrak{M}\left\langle\varepsilon^{\prime \prime}\right\rangle\right)$ for some $\varepsilon^{\prime}, \varepsilon^{\prime \prime} \succcurlyeq \varepsilon^{*} \mid w^{*}$. Then we have $\tau\left(a, 3^{s-1}\right)=\tau\left(b, 3^{s-1}\right)$, and $\tau H\left(a, 3^{s-1}, s^{*}\right)=\tau H\left(b, 3^{s-1}, s^{*}\right)$.

(b) For any $k \in \mathbb{N}$ such that $0<k \leqslant s$, each local type $\theta \in \operatorname{TY}\left(3^{k-1}\right)$ is either limited or $s^{*}$-unlimited in any horizontal type $\varphi$ realized in the models of the class of models (11.1).

(c) For any $k \in \mathbb{N}$ such that $0<k \leqslant s$, each horizontal type $\varphi \in \operatorname{TYH}\left(3^{k-1}, s^{*}\right)$ is either limited or $s^{*}$-unlimited in the class of models (11.1).

Proof. Part (a) immediately follows by the description of the neighborhoods of the point $E$ in Lemmas 9.8-9.10 together with the inequalities (a) and (b) of Lemma 9.13. And part (b) follows directly by the definition of the horizontal types $\varphi \in \operatorname{TYH}\left(3^{k-1}, s^{*}\right)$.

(c) Let a type $\varphi \in \operatorname{TYH}\left(3^{k-1}, s^{*}\right)$ be given. Consider the set $\psi_{1}, \psi_{2}, \ldots, \psi_{n} \in$ $\operatorname{TYH}\left(3^{s-1}, s^{*}\right)$ of all horizontal types of larger radius $3^{s-1}$ satisfying the condition that $\varphi$ is the restriction of some $\psi_{i}, 1 \leqslant i \leqslant n$ to the smaller radius $3^{k-1}$. One can easily see that each $H$-class of any model of the class (11.1) which satisfies $\varphi$ must be of one of types $\psi_{1}, \psi_{2}, \ldots, \psi_{n}$. By Lemma 10.18, each of these horizontal types is either limited or $s^{*}$-unlimited in the class of models (11.1). In the case when at least one type $\psi_{j}, 1 \leqslant j \leqslant n$, is $s^{*}$-unlimited, the type $\varphi$ will be $s^{*}$-unlimited in the class of models (11.1). In the case when all the types $\psi_{1}, \psi_{2}, \ldots, \psi_{n}$ are limited in the class of models (11.1), each of these types must occur a fixed number of times. Whether the type $\varphi$ is limited depends on the sum of the number of occurrences of the types $\psi_{1}, \psi_{2}, \ldots, \psi_{n}$.

Now we turn directly to the Ehrenfeucht back-and-forth method. Suppose we have a family of relations $\equiv_{k}, 0 \leqslant k \leqslant s$, between pairs of tuples of equal length $\left(a_{0}, \ldots, a_{n}\right)$ and $\left(b_{0}, \ldots, b_{n}\right)$ of the models $\mathfrak{M}\left\langle\varepsilon^{\prime}\right\rangle$ and $\mathfrak{M}\left\langle\varepsilon^{\prime \prime}\right\rangle$ with $a_{i} \in \mathfrak{M}\left\langle\varepsilon^{\prime}\right\rangle$, $b_{j} \in \mathfrak{M}\left\langle\varepsilon^{\prime \prime}\right\rangle$, and $n=s-k$. We say that this family of relations satisfies the Ehrenfeucht conditions on steps if the following properties hold:

(E0) $\left(\exists a \in \mathfrak{M}\left\langle\varepsilon^{\prime}\right\rangle\right)\left(\exists b \in \mathfrak{M}\left\langle\varepsilon^{\prime \prime}\right\rangle\right)\left[(a) \equiv_{s}(b)\right]$;

(E1) if $0<k \leqslant s$ and $n=s-k$ then 


$$
\begin{aligned}
& \left(a_{0}, a_{1}, \ldots, a_{n}\right) \equiv_{k}\left(b_{0}, b_{1}, \ldots, b_{n}\right) \Rightarrow \\
& \left(\forall a \in \mathfrak{M}\left\langle\varepsilon^{\prime}\right\rangle\right)\left(\exists b \in \mathfrak{M}\left\langle\varepsilon^{\prime \prime}\right\rangle\right)\left[\left(a_{0}, a_{1}, \ldots, a_{n}, a\right) \equiv_{k-1}\left(b_{0}, b_{1}, \ldots, b_{n}, b\right)\right]
\end{aligned}
$$

(E2) if $0<k \leqslant s$ and $n=s-k$ then

$$
\begin{aligned}
& \left(a_{0}, a_{1}, \ldots, a_{n}\right) \equiv_{k}\left(b_{0}, b_{1}, \ldots, b_{n}\right) \Rightarrow \\
& \left(\forall b \in \mathfrak{M}\left\langle\varepsilon^{\prime \prime}\right\rangle\right)\left(\exists a \in \mathfrak{M}\left\langle\varepsilon^{\prime}\right\rangle\right)\left[\left(a_{0}, a_{1}, \ldots, a_{n}, a\right) \equiv_{k-1}\left(b_{0}, b_{1}, \ldots, b_{n}, b\right)\right] .
\end{aligned}
$$

(E3) if $\left(a_{0}, a_{1}, \ldots, a_{s}\right) \equiv_{0}\left(b_{0}, b_{1}, \ldots, b_{s}\right)$, then the map $\lambda$ which is defined by $\lambda\left(a_{i}\right)=b_{i}$ is an isomorphism between the atomic diagrams $A D\left(a_{0}, a_{1}, \ldots a_{s}\right)$ and $A D\left(b_{0}, b_{1}, \ldots b_{s}\right)$ in $\mathfrak{M}\left\langle\varepsilon^{\prime}\right\rangle$ and $\mathfrak{M}\left\langle\varepsilon^{\prime \prime}\right\rangle$, respectively.

The following statement expresses the essence of the Ehrenfeucht back-andforth method.

Lemma 11.2. [Ehrenfeucht Lemma] Suppose that for the models $\mathfrak{M}\left\langle\varepsilon^{\prime}\right\rangle$ and $\mathfrak{M}\left\langle\varepsilon^{\prime \prime}\right\rangle$, there is a family of relations $\equiv_{k}, 0 \leqslant k \leqslant s$, between tuples of these models such that the Ehrenfeucht conditions (E0)-(E3) hold for a given $s>0$. Then $\mathfrak{M}\left\langle\varepsilon^{\prime}\right\rangle \equiv_{s} \mathfrak{M}\left\langle\varepsilon^{\prime \prime}\right\rangle$ holds. (That is, $\mathfrak{M}\left\langle\varepsilon^{\prime}\right\rangle$ and $\mathfrak{M}\left\langle\varepsilon^{\prime \prime}\right\rangle$ satisfy the same sentences of quantifier rank $\leqslant$ s.)

Proof. By standard methods (see Hodges [8]). Note that the condition (E0) is just auxiliary to start the back-and-forth process. The essential backand-forth steps are ensured by the conditions (E1) and (E2).

For any two models $\mathfrak{M}\left\langle\varepsilon^{\prime}\right\rangle$ and $\mathfrak{M}\left\langle\varepsilon^{\prime \prime}\right\rangle$ of the class (11.1), we define the relations $\equiv_{k}, 0 \leqslant k \leqslant s$, between pairs of tuples of equal length $\left(a_{0}, \ldots, a_{n}\right)$ and $\left(b_{0}, \ldots, b_{n}\right)$ from $\mathfrak{M}\left\langle\varepsilon^{\prime}\right\rangle$ and $\mathfrak{M}\left\langle\varepsilon^{\prime \prime}\right\rangle, n \in \mathbb{N}, a_{i} \in \mathfrak{M}\left\langle\varepsilon^{\prime}\right\rangle, b_{j} \in \mathfrak{M}\left\langle\varepsilon^{\prime \prime}\right\rangle, n=s-k$, as follows. We set

$$
\left(a_{0}, a_{1}, \ldots a_{n}\right) \equiv_{0}\left(b_{0}, b_{1}, \ldots b_{n}\right), \text { for } n=s,
$$

if the mapping $\lambda\left(a_{i}\right)=b_{i}$ is an isomorphism between the atomic diagrams $A D\left(a_{0}, a_{1}, \ldots a_{n}\right)$ and $A D\left(b_{0}, b_{1}, \ldots b_{n}\right)$ in $\mathfrak{M}\left\langle\varepsilon^{\prime}\right\rangle$ and $\mathfrak{M}\left\langle\varepsilon^{\prime \prime}\right\rangle$, respectively. Also, we set

$$
\left(a_{0}, a_{1}, \ldots a_{n}\right) \equiv_{k}\left(b_{0}, b_{1}, \ldots b_{n}\right), \text { for } 0<k \leqslant s, n=s-k,
$$

if the following five conditions hold:

(A) $a_{0}=E\left(\mathfrak{M}\left\langle\varepsilon^{\prime}\right\rangle\right)$, and $b_{0}=E\left(\mathfrak{M}\left\langle\varepsilon^{\prime \prime}\right\rangle\right)$;

(B) $\tau\left(a_{i}, 3^{k-1}\right)=\tau\left(b_{i}, 3^{k-1}\right)$, for $i=1, \ldots, n$;

(C) $\tau H\left(a_{i}, 3^{k-1}, s^{*}\right)=\tau H\left(b_{i}, 3^{k-1}, s^{*}\right)$, for $i=1, \ldots, n$;

(D) $S_{u} N_{v}\left(a_{i}, a_{j}\right) \leftrightarrow S_{u} N_{v}\left(b_{i}, b_{j}\right)$, for all $i, j \in\{0,1, \ldots, n\}$ and all $u, v \in \mathbb{Z}$ such that $|u|+|v| \leqslant 2 \cdot 3^{k-1}$;

(E) $D_{u}\left(a_{i}, a_{j}\right) \leftrightarrow D_{u}\left(b_{i}, b_{j}\right)$, for all $i, j \in\{0,1, \ldots, n\}$ and all $u \in \mathbb{Z}$ such that $|u| \leqslant 2 \cdot 3^{k-1}$.

The following lemma states some basic properties which follow immediately from this definition. 
Lemma 11.3. Consider tuples $\left(a_{0}, a_{1}, \ldots, a_{n}\right)$ and $\left(b_{0}, b_{1}, \ldots, b_{n}\right)$ in models $\mathfrak{M}\left\langle\varepsilon^{\prime}\right\rangle$ and $\mathfrak{M}\left\langle\varepsilon^{\prime \prime}\right\rangle, \varepsilon^{\prime}, \varepsilon^{\prime \prime} \succcurlyeq \varepsilon^{*} \mid w^{*}$, for which the relation

$$
\left(a_{0}, a_{1}, \ldots, a_{n}\right) \equiv_{k}\left(b_{0}, b_{1}, \ldots, b_{n}\right)
$$

defined above holds, where $0<k \leqslant s, n=s-k$. Then the following assertions hold:

(a) $\tau\left(a_{i}, 3^{k-1}\right)=\tau\left(b_{i}, 3^{k-1}\right)$, for $i=0,1, \ldots, n$.

(b) $\tau H\left(a_{i}, 3^{k-1}, s^{*}\right)=\tau H\left(b_{i}, 3^{k-1}, s^{*}\right)$, for $i=0,1, \ldots, n$.

(c) Suppose $k \geqslant 2$. Let $m \in \mathbb{Z}$ be such that $|m| \leqslant 2 \cdot 3^{k-2}$, and let arbitrary elements $a \in \mathfrak{M}\left\langle\varepsilon^{\prime}\right\rangle$ and $b \in \mathfrak{M}\left\langle\varepsilon^{\prime \prime}\right\rangle$ be given such that $D_{m}\left(a_{u}, a\right)$ and $D_{m}\left(b_{u}, b\right)$ hold for some $u \in\{0,1, \ldots, n\}$. Then $\tau H\left(a, 3^{k-2}, s^{*}\right)=\tau H\left(b, 3^{k-2}, s^{*}\right)$.

(d) Suppose $k \geqslant 2$. Let $t \in \mathbb{Z}$ be such that $|t| \leqslant 2 \cdot 3^{k-2}$, and let arbitrary elements $a \in \mathfrak{M}\left\langle\varepsilon^{\prime}\right\rangle$ and $b \in \mathfrak{M}\left\langle\varepsilon^{\prime \prime}\right\rangle$ be given such that $D_{t}\left(a_{u}, a\right)$ and $D_{t}\left(b_{u}, b\right)$ hold for some $u \in\{0,1, \ldots, n\}$. Then, for any local type $\theta \in T Y\left(3^{k-2}\right)$, the number of points realizing $\theta$ in the set $\left\{x \mid H(a, x) \& \rho\left(x,\left\{a_{0}, a_{1}, \ldots, a_{n}\right\}\right) \leqslant 2\right.$. $\left.3^{k-2}\right\}$ of $\mathfrak{M}\left\langle\varepsilon^{\prime}\right\rangle$ is exactly equal to the number of points realizing $\theta$ in the set $\left\{z \mid H(b, z) \& \rho\left(z,\left\{b_{0}, b_{1}, \ldots, b_{n}\right\}\right) \leqslant 2 \cdot 3^{k-2}\right\}$ of $\mathfrak{M}\left\langle\varepsilon^{\prime \prime}\right\rangle$.

(e) Suppose $k \geqslant 2$. For any horizontal type $\varphi \in T Y H\left(3^{k-2}, s^{*}\right)$, the number of $H$-classes realizing $\varphi$ in the set $\left\{x \mid \rho^{*}\left(x,\left\{a_{0}, a_{1}, \ldots, a_{n}\right\}\right) \leqslant 2 \cdot 3^{k-2}\right\}$ of $\mathfrak{M}\left\langle\varepsilon^{\prime}\right\rangle$ is exactly equal to the number of $H$-classes realizing $\varphi$ in the set $\left\{z \mid \rho^{*}\left(z,\left\{b_{0}, b_{1}, \ldots, b_{n}\right\}\right) \leqslant 2 \cdot 3^{k-2}\right\}$ of $\mathfrak{M}\left\langle\varepsilon^{\prime \prime}\right\rangle$.

Proof. (a), (b) The identity of the types of the elements $a_{0}$ and $b_{0}$ in the case $k=s$ follows directly from condition (A) together with Lemma 11.1(a), while in the case $k<s$, we also use Lemmas 10.2 and 10.7. The equality of the types of the other pairs $a_{i}, b_{i}, i>0$, is immediate by the definition of the relations $\equiv_{k}$.

(c) First, we consider a simpler case, when both the conditions $D_{m}\left(a_{u}, a\right)$ and $D_{m}\left(b_{u}, b\right)$ hold for some $m$ together with the condition $0 \leqslant m \leqslant 2 \cdot 3^{k-2}$. Then the equality of the types $\tau H\left(a, 3^{k-2}, s^{*}\right)=\tau H\left(b, 3^{k-2}, s^{*}\right)$ follows by Lemma 10.8 and part (b) of this lemma.

In the other case, when both conditions $D_{m}\left(a, a_{u}\right)$ and $D_{m}\left(b, b_{u}\right)$ hold for a parameter $m$ in the interval $0<m \leqslant 2 \cdot 3^{k-2}$, the following difficulty arises. The horizontal types of the elements $a$ and $b$ should be defined by the type $\varphi=\tau H\left(a_{u}, 3^{k-1}, s^{*}\right)=\tau H\left(b_{u}, 3^{k-1}, s^{*}\right)$, based on Lemma 10.9 , because it is applicable to some neighborhood below the places where the elements $a_{u}$ and $b_{u}$ are located, but only under the additional conditions stated there. If $m \notin$ $\left[3^{k-2}+1,2 \cdot 3^{k-2}\right]$ holds, or $m \in\left[3^{k-2}+1,2 \cdot 3^{k-2}\right]$ holds, and, at the same time at least one of the conditions (10.1) or (10.2) is true with $a=a_{u}$ and $b=b_{u}$, then, by Lemma 10.9 , since the horizontal $\left(3^{k-1}, s^{*}\right)$-types of the elements $a_{u}$ and $b_{u}$ are identical, they completely determine the $\left(3^{k-2}, s^{*}\right)$-types of the elements $a$ 
and $b$, which also will be identical. It remains to consider only the situation, when the conditions of Lemma 10.9 are not applicable. In this case, we have the following sequence of assertions:

$$
\begin{aligned}
& m \in\left[3^{k-2}+1,2 \cdot 3^{k-2}\right], \\
& \left(\exists t \in\left[0.5\left(3^{k-1}+3\right), m+0.5\left(3^{k-2}+1\right)\right]\right)\left[D_{t}\left(E, a_{u}\right) \vee D_{t}\left(E, b_{u}\right)\right], \\
& (10.2) \text { is false with } a=a_{u}, b=b_{u} .
\end{aligned}
$$

One can see that (11.3) is just equivalent to the negation of condition (10.1) with $a=a_{u}$ and $b=b_{u}$. According to requirement (A) for $\equiv_{k}$, the special oneelement region $E$ in the models $\mathfrak{M}\left\langle\varepsilon^{\prime}\right\rangle$ and $\mathfrak{M}\left\langle\varepsilon^{\prime \prime}\right\rangle$ is represented by the elements $a_{0}$ and $b_{0}$. Thus, (11.3) means that one of the following two conditions holds:

$$
\begin{aligned}
& D_{t}\left(a_{0}, a_{u}\right), \text { for some } t \in\left[0.5\left(3^{k-1}+3\right), m+0.5\left(3^{k-2}+1\right)\right], \\
& D_{t}\left(b_{0}, b_{u}\right), \text { for some } t \in\left[0.5\left(3^{k-1}+3\right), m+0.5\left(3^{k-2}+1\right)\right] .
\end{aligned}
$$

Suppose that (11.5) holds. Since the parameter $t$ in (11.5) is a positive number and does not exceed $2 \cdot 3^{k-1}$, we have by condition (E) for $\equiv_{k}$ that $D_{t}\left(a_{0}, a_{u}\right) \leftrightarrow$ $D_{t}\left(b_{0}, b_{u}\right)$. From this, we obtain:

$$
\begin{aligned}
& D_{t}\left(a_{0}, a_{u}\right) \& D_{t}\left(b_{0}, b_{u}\right) \\
& \text { for some } t \in\left[0.5\left(3^{k-1}+3\right), m+0.5\left(3^{k-2}+1\right)\right] \text {. }
\end{aligned}
$$

In the other case, when condition (11.6) is true, one can give a symmetric argument.

In this situation, the position of the elements $a$ and $b$ with respect to $a_{u}$ and $b_{u}$ is given, and also, bounds on the position of the elements $a_{0}$ and $b_{0}$ with respect to $a_{u}$ and $b_{u}$ are given. Combining these conditions, we obtain the following relations between $a_{0}, b_{0}$, and $a, b$ :

$$
\begin{aligned}
& D_{i}\left(a_{0}, a\right), D_{i}\left(b_{0}, b\right), \\
& i=t-m, \\
& -0.5\left(3^{k-2}-3\right) \leqslant i \leqslant 0.5\left(3^{k-2}+1\right) .
\end{aligned}
$$

Thus, the elements $a$ and $b$ are located in some neighborhood of the elements $a_{0}$ and $b_{0}$ at the same $D$-distance. Since the number $i$ in (11.8) satisfies the condition $|i| \leqslant 2 \cdot 3^{k-2}$, the horizontal $\left(3^{k-2}, s^{*}\right)$-types of the elements $a$ and $b$ are uniquely defined by the $\left(3^{k-1}, s^{*}\right)$-types of the elements $a_{0}$ and $b_{0}$. If we have $i \geqslant 0$, Lemma 10.8 can be used. In the other case $i<0$, we have the following situation with a new parameter $j=-i$ :

$$
D_{j}\left(a, a_{0}\right), D_{j}\left(b, b_{0}\right), \quad 0<j \leqslant 0.5\left(3^{k-2}-3\right) .
$$


One can now see that $j \notin\left[3^{k-2}+1,2 \cdot 3^{k-2}\right]$, and consequently, it is possible to use Lemma 10.9 avoiding its area of exclusion. As a result, by applying one of the Lemmas 10.8 and 10.9, we obtain that the horizontal $\left(3^{k-2}, s^{*}\right)$-types of the elements $a$ and $b$ must be identical.

Summarizing the proof of property (c), one can briefly say that if for some $u \in\{1, \ldots, n\}$, the definition of the horizontal types of the new elements $a$ and $b$ by the horizontal types of the elements $a_{u}$ and $b_{u}$ puts us into the area of exclusion of Lemma 10.9, it will be possible to define the types of these elements $a$ and $b$ in some other way, namely, by the types of the elements $a_{0}$ and $b_{0}$, either using Lemma 10.8, or using Lemma 10.9, avoiding its area of exclusion.

Part (d) follows from Lemma 7.2 and Lemma 10.3, taking into account that the adjoining spheres of radius $3^{k-1}$ of the elements $a_{i}$ in the model $\mathfrak{M}\left\langle\varepsilon^{\prime}\right\rangle$ and the elements $b_{i}$ in the model $\mathfrak{M}\left\langle\varepsilon^{\prime \prime}\right\rangle$ are rigidly linked in identical positions in these two models because of condition (D) in the definition of $\equiv_{k}$.

Part (e) follows from Lemma 11.3 (c), taking into account that the adjoining neighborhoods of $\rho^{*}$-radius $3^{k-1}$ of the elements $a_{i}$ in the model $\mathfrak{M}\left\langle\varepsilon^{\prime}\right\rangle$ and the elements $b_{i}$ in the model $\mathfrak{M}\left\langle\varepsilon^{\prime \prime}\right\rangle$ are rigidly linked in identical positions in these two models by condition (E) in the definition of $\equiv_{k}$.

We prove now the Main Statement that the family of relations $\equiv_{k}, 0 \leqslant k \leqslant s$, satisfies the Ehrenfeucht conditions on $s$ steps:

Lemma 11.4. For the family of relations $\equiv_{k}, 0 \leqslant k \leqslant s$, introduced between tuples from models $\mathfrak{M}\left\langle\varepsilon^{\prime}\right\rangle$ and $\mathfrak{M}\left\langle\varepsilon^{\prime}\right\rangle$ with $\varepsilon^{\prime}, \varepsilon^{\prime \prime} \succcurlyeq \varepsilon^{*} \uparrow w^{*}$, the requirements (E0)-(E3) on steps hold.

Proof. Let $\mathfrak{M}\left\langle\varepsilon^{\prime}\right\rangle$ and $\mathfrak{M}\left\langle\varepsilon^{\prime \prime}\right\rangle$ be two arbitrary models of the class of models (11.1). The fact that condition (E0) holds follows directly by Lemma 11.1 (a) and the fact that condition (E3) holds follows directly from the definition of $\equiv_{0}$.

For the conditions (E1) and (E2), it is enough to check only one of them by symmetry. Without loss of generality, we consider condition (E1). We consider any $k, 0<k \leqslant s$, and arbitrary tuples $\left(a_{0}, a_{1}, \ldots, a_{n}\right)$ and $\left(b_{0}, b_{1}, \ldots, b_{n}\right)$ with $n=s-k$ for which the condition $\left(a_{0}, a_{1}, \ldots, a_{n}\right) \equiv_{k}\left(b_{0}, b_{1}, \ldots, b_{n}\right)$ holds. That is, conditions $(\mathrm{A})-(\mathrm{E})$ above hold for this pair of tuples. Also, let $a$ be an arbitrary element of $\mathfrak{M}\left\langle\varepsilon^{\prime}\right\rangle$. We have to find an element $b \in \mathfrak{M}\left\langle\varepsilon^{\prime \prime}\right\rangle$ such that $\left(a_{0}, a_{1}, \ldots, a_{n}, a\right) \equiv_{k-1}\left(b_{0}, b_{1}, \ldots, b_{n}, b\right)$ holds.

We split the analysis into three cases depending on the relative position of $a$ with respect to the elements $a_{0}, a_{1}, \ldots, a_{n}$.

CASE 1. $\rho\left(a_{u}, a\right) \leqslant 2 \cdot 3^{k-2}$ holds for some $u \in\{0,1, \ldots, n\}$. In this case, we find parameters $i, j \in \mathbb{Z}$ such that $|i|+|j|=\rho\left(a_{u}, a\right)$ and $S_{i} N_{j}\left(a_{u}, a\right)$ holds. Given $a \in \mathfrak{M}\left\langle\varepsilon^{\prime}\right\rangle$, we choose an element $b \in \mathfrak{M}\left\langle\varepsilon^{\prime \prime}\right\rangle$ such that $S_{i} N_{j}\left(b_{u}, b\right)$ holds. The existence of such an element $b$ follows from Lemma 10.1. If there is more than one index $u$ with this condition $\rho\left(a_{u}, a\right) \leqslant 2 \cdot 3^{k-2}$, all such elements $a_{u}$ will 
be rigidly "linked" to each other by conditions of the form (D) in the definition $\equiv_{k}$, because the distances between any two such elements $a_{u}$ are limited by the number $2 \cdot\left(2 \cdot 3^{k-2}\right)<2 \cdot 3^{k-1}$, due to the triangle inequality $(7.2)$ with the intermediate element $a$. Therefore, the choice of such an element $b$ is unique, i.e., it does not depend on the $u$ above.

The following reasoning shows that we obtain

$$
\left(a_{0}, a_{1}, \ldots, a_{n}, a\right) \equiv_{k-1}\left(b_{0}, b_{1}, \ldots, b_{n}, b\right) .
$$

Namely, condition (A) holds by induction, and both conditions (D) and (E) are guaranteed by the construction. Condition (B) for $\equiv_{k-1}$ with the pair $a$ and $b$ is ensured by Lemma 10.3 , while for the other pairs $a_{i}$ and $b_{i}, 0 \leqslant i \leqslant n$, it is a consequence of the corresponding requirements for $\equiv_{k}$ by virtue of Lemma 10.2. Now we proceed to condition (C), which we verify only for $k \geqslant 2$ (the case $k=1$ is considered later separately). For pairs $a_{i}$ and $b_{i}, 0 \leqslant i \leqslant n$, condition (C) for $\equiv_{k-1}$ is a consequence of the corresponding requirement for $\equiv_{k}$ by Lemma 10.7. Now we consider condition (C) for the pair $a$ and $b$. From the relation (7.3) between the distance and quasidistance, we obtain that $\rho^{*}\left(a_{u}, a\right)=$ $\rho^{*}\left(b_{u}, b\right) \leqslant 2 \cdot 3^{k-2}$, namely, we have $D_{j}\left(a_{u}, a\right) \& D_{j}\left(b_{u}, b\right)$ from the number $j$ chosen at the beginning of Case 1 . Since $|j| \leqslant 2 \cdot 3^{k-2}$ holds, it follows by the equality of the horizontal $\left(3^{k-1}, s^{*}\right)$-types of the elements $a_{u}$ and $b_{u}$ that $\tau H\left(a, 3^{k-2}, s^{*}\right)=\tau H\left(b, 3^{k-2}, s^{*}\right)$ by Lemma $11.3(\mathrm{c})$.

CASE 2. $\rho^{*}\left(a_{u}, a\right) \leqslant 2 \cdot 3^{k-2}$ holds for some $u \in\{0,1, \ldots, n\}$, and $\rho\left(a_{v}, a\right)>$ $2 \cdot 3^{k-2}$ holds for all $v \in\{0,1, \ldots, n\}$. In this case, we find a number $i$ with $|i|=$ $\rho^{*}\left(a_{u}, a\right)$ for which $D_{i}\left(a_{u}, a\right)$ holds. Given $a \in \mathfrak{M}\left\langle\varepsilon^{\prime}\right\rangle$, we first find an element $b^{\prime} \in \mathfrak{M}\left\langle\varepsilon^{\prime \prime}\right\rangle$ such that $D_{i}\left(b_{u}, b^{\prime}\right)$ holds. The existence of such a $b^{\prime}$ immediately follows from Lemma 10.6, taking into consideration that $\tau H\left(a_{u}, 3^{k-1}, s^{*}\right)=$ $\tau H\left(b_{u}, 3^{k-1}, s^{*}\right)$ by Lemma $11.3(\mathrm{~b})$. If there is more than one index $u$ with the condition $\rho^{*}\left(a_{u}, a\right) \leqslant 2 \cdot 3^{k-2}$, all such elements $a_{u}$ will be rigidly "linked" to each other by the conditions (E) in the definition of $\equiv_{k}$ because the distance $\rho^{*}$ between any of two such elements $a_{u}$ is limited within the number $2 \cdot(2$. $\left.3^{k-2}\right)<2 \cdot 3^{k-1}$ by the triangle inequality (7.1) with the intermediate element $a$. Therefore, the choice of this element $b^{\prime}$ is unique up to $H$-class, that is, the class $\left[b^{\prime}\right]_{H}$ does not depend on the $u$ which meets the conditions above. The horizontal $\left(3^{k-1}, s^{*}\right)$-types of the elements $a_{u}$ and $b_{u}$ are identical by Lemma $11.3(\mathrm{~b})$. Then, by Lemma $11.3(\mathrm{c})$, the horizontal types $\tau H\left(a, 3^{k-2}, s^{*}\right)$ and $\tau H\left(b^{\prime}, 3^{k-2}, s^{*}\right)$ will be identical as well.

Once the choice of $b^{\prime}$ is made, we choose an element $b \in\left[b^{\prime}\right]_{H}$ satisfying the conditions $\tau\left(b, 3^{k-2}\right)=\tau\left(a, 3^{k-2}\right)$, and $\rho\left(b_{v}, b\right)>2 \cdot 3^{k-2}$ for all $v \in$ $\{0,1, \ldots, n\}$. One can prove the existence of such an element $b$ as follows. Let $\varphi=\tau H\left(a, 3^{k-2}, s^{*}\right)$. If the local type $\theta=\tau\left(a, 3^{k-2}\right)$ is limited in the horizontal type $\varphi$, then by definition it is realized by the same number of elements in every $H$-class of this type $\varphi$ in the models of the class (11.1). In this case, we appeal 
to Lemma 11.3 (d) for the choice of $b \in\left[b^{\prime}\right]_{H}$. Namely, by this lemma, the two sets

$$
\begin{aligned}
& \left\{x \mid H(a, x) \& \rho\left(x,\left\{a_{0}, a_{1}, \ldots, a_{n}\right\}\right) \leqslant 2 \cdot 3^{k-2}\right\}, \\
& \left\{z \mid H\left(b^{\prime}, z\right) \& \rho\left(z,\left\{b_{0}, b_{1}, \ldots, b_{n}\right\}\right) \leqslant 2 \cdot 3^{k-2}\right\},
\end{aligned}
$$

contain an equal number of realizations of the type $\theta$; therefore, there will also be an equal number of realizations of the type $\theta$ in the two sets

$$
\begin{aligned}
& \left\{x \mid H(a, x) \& \rho\left(x,\left\{a_{0}, a_{1}, \ldots, a_{n}\right\}\right)>2 \cdot 3^{k-2}\right\}, \\
& \left\{z \mid H\left(b^{\prime}, z\right) \& \rho\left(z,\left\{b_{0}, b_{1}, \ldots, b_{n}\right\}\right)>2 \cdot 3^{k-2}\right\} .
\end{aligned}
$$

By assumption, our element $a$ realizes the type $\theta$; moreover, $a$ belongs to the set (11.9). From this it follows that a realization of the type $\theta$ must exist in the set (11.10) as well, which guarantees the existence of the element $b \in\left[b^{\prime}\right]_{H}$ with the above conditions. In the other case, when the local type $\theta$ is $s^{*}$-unlimited in the horizontal type $\varphi$, by definition, the number of realizations of this type $\theta$ in every $H$-class of type $\varphi$ in any model of the class (11.1) is not less than $s^{*}$. Then a realization of type $\theta$ in the set (11.10) must exist by Lemma 9.1 (a).

Once the choice of an element $b \in\left[b^{\prime}\right]_{H}$ is made, it is necessary to prove that we will have $\left(a_{0}, a_{1}, \ldots, a_{n}, a\right) \equiv_{k-1}\left(b_{0}, b_{1}, \ldots, b_{n}, b\right)$. Condition (A) for $\equiv_{k-1}$ holds by induction, conditions (D) and (E) are guaranteed immediately by the choice of $b$ and $b^{\prime}$. Conditions (B) and (C) for $a$ and $b$ were considered above, while for the other pairs $a_{i}$ and $b_{i}, 0 \leqslant i \leqslant n$, they are true by induction because of Lemma 10.2 and Lemma 10.7 .

CASE 3. $\rho^{*}\left(a_{v}, a\right)>2 \cdot 3^{k-2}$ holds for all $v \in\{0,1, \ldots, n\}$. Then also the condition $\rho\left(a_{v}, a\right)>2 \cdot 3^{k-2}$ will hold for all $v \in\{0,1, \ldots, n\}$. In this case, we first find an element $b^{\prime} \in \mathfrak{M}\left\langle\varepsilon^{\prime \prime}\right\rangle$ such that $\tau H\left(a, 3^{k-2}, s^{*}\right)=\tau H\left(b^{\prime}, 3^{k-2}, s^{*}\right)$, and $\rho^{*}\left(b_{v}, b^{\prime}\right)>2 \cdot 3^{k-2}$ for all $v \in\{0,1, \ldots, n\}$ holds. The existence of such an element $b^{\prime}$ can be proved by Lemma $11.1(\mathrm{c})$ as follows. In the case when the horizontal type $\varphi=\tau H\left(a, 3^{k-2}, s^{*}\right)$ is limited on the class of models (11.1), it is realized by definition by the same number of the $H$-classes in any model of the class (11.1). By Lemma 11.3(e), in the neighborhood of the elements $a_{0}, a_{1}, \ldots, a_{n}$ and $b_{0}, b_{1}, \ldots, b_{n}$ at a distance $\rho^{*}$ not bigger than $2 \cdot 3^{k-2}$ from these elements, there is the same number of $H$-classes of the type $\varphi$ in both models $\mathfrak{M}\left\langle\varepsilon^{\prime}\right\rangle$ and $\mathfrak{M}\left\langle\varepsilon^{\prime \prime}\right\rangle$. Then there must also be an equal number of $H$-classes of this type $\varphi$ in the complements

$$
\begin{aligned}
& \left\{x \mid \rho^{*}\left(x,\left\{a_{0}, a_{1}, \ldots, a_{n}\right\}\right)>2 \cdot 3^{k-2}\right\}, \\
& \left\{y \mid \rho^{*}\left(y,\left\{b_{0}, b_{1}, \ldots, b_{n}\right\}\right)>2 \cdot 3^{k-2}\right\} .
\end{aligned}
$$

By the condition, the element $a$ in the model $\mathfrak{M}\left\langle\varepsilon^{\prime}\right\rangle$ realizes the type $\varphi$ in the region (11.11), therefore, there must also be a realization of the type $\varphi$ in the area (11.12) of the model $\mathfrak{M}\left\langle\varepsilon^{\prime \prime}\right\rangle$, which guarantees the existence of an element 
$b^{\prime}$ with the above conditions. In the other case, when the type $\varphi$ is $s^{*}$-unlimited on the class of models (11.1), the number of $H$-classes of type $\varphi$ in each of these models must be not less than $s^{*}$, and therefore the existence of such an element $b^{\prime}$ of type $\varphi$ in the set (11.12) is ensured by Lemma 9.1 (b).

Once an element $b^{\prime}$ is chosen, we proceed to the choice of an element $b \in$ $\mathfrak{M}\left\langle\varepsilon^{\prime \prime}\right\rangle$ as follows. We choose an element $b \in\left[b^{\prime}\right]_{H}$ with $\tau\left(b, 3^{k-2}\right)=\tau\left(a, 3^{k-2}\right)$. The existence of such an element $b$ follows from the identity of the horizontal $\left(3^{k-2}, s^{*}\right)$-types of the elements $a$ and $b^{\prime}$, which guarantees the coincidence of the local types contained inside them. As a result, we obtain $\left(a_{0}, a_{1}, \ldots, a_{n}, a\right) \equiv_{k-1}$ $\left(b_{0}, b_{1}, \ldots, b_{n}, b\right)$, which can be proved as follows. Condition (A) for $\equiv_{k-1}$ holds by induction, and conditions (D) and (E) are guaranteed by the choice of $b$ and $b^{\prime}$. Conditions (B) and (C) for $a$ and $b$ are ensured by the choice of $b$ and $b^{\prime}$ as well, while for the other pairs $a_{i}$ and $b_{i}, 0 \leqslant i \leqslant n$, they are true by induction by Lemma 10.2 and Lemma 10.7.

It remains to note that in the case $k=1$, when the relation $\equiv_{k-1}$ is defined by a simpler condition (namely, the identity of atomic diagrams) instead of conditions (A) - (E), the above argument is not applicable any more. Nevertheless, in this case, the truth of the back-and-forth condition (E1) can also be established. For the proof, three cases must again be considered, similar to those discussed above, by a similar but simpler reasoning. The choice of the element $b \in \mathfrak{M}\left\langle\varepsilon^{\prime \prime}\right\rangle$, given $a \in \mathfrak{M}\left\langle\varepsilon^{\prime}\right\rangle$, is ensured as above (with some obvious simplifications). Instead of conditions (A) - (E), we need to check the identity of the atomic diagrams of the tuples $\left(a_{0}, a_{1}, \ldots, a_{n}, a\right)$ and $\left(b_{0}, b_{1}, \ldots, b_{n}, b\right)$, which is easy by Lemma 7.1.

Thus, Lemma 11.4 is completely proved.

Lemma 11.5 For models of the theory T, property (4.25) holds.

Proof. Immediate by Lemmas 11.2 and 11.4.

So the proof of Theorem 1.5 is completely finished. 


\section{References}

1. C. C. Chang, and H. J. Keisler, Model Theory, North-Holland, AmsterdamNew York, 1990.

2. S. S. Goncharov, Certain properties of the constructivization of boolean algebras, Siberian. Math. J., v. 16, No. 2, 1975, p. 264-278 (Russian).

3. S. S. Goncharov, Countable Boolean Algebras and Decidability, Plenum, New York, 1997.

4. S. S. Goncharov and Yu.L. Ershov, Constructive models, Plenum, New York, 1999.

5. W. HAnF, Model-theoretic methods in the study of elementary logic, Symposium on the Theory of Models, North-Holland, Amsterdam, 1965, p. 132-145.

6. W. Hanf, Primitive Boolean algebras, Proc. Sympos. Pure Math., 1974, p. 75-90.

7. W. Hanf, The Boolean algebra of Logic, Bull. American Math. Soc., v. 81, 1975, p. $587-589$.

8. W. Hodges, A shorter model theory, Cambridge University Press, Cambridge, 1997.

9. C. P. Odintsov and V.L. Selivanov, Arithmetical hierarchy and ideals of numerated Boolean algebras, Siberian Math. J., v.30, No.6, 1989, p.140-149.

10. M. G. PeRETyAT'KIn, Strongly constructive models and numerations of the boolean algebra of recursive sets, Alg. Logika, v. 10, No. 5, 1971, p. 535-557 (Russian).

11. M. G. Peretyat'Kin, Finitely axiomatizable theories, Plenum, New York, 1997.

12. M. G. Peretyat'KIn, Finitely axiomatizable theories and Lindenbaum algebras of semantic classes, in: "Computability Theory and Its Applications: Current Trends and Open Problems", Amer. Math. Soc., Providence, R. I., to appear.

13. M. G. PERETyAT'KIn, Signature reduction method for finitely axiomatizable theories, to appear.

14. V. L. Selivanov, Universal Boolean algebras and their applications, International Conference on Algebra (abstracts), Russia, Barnaul, 20-25 August 1991, p. 127.

15. V. L. Selivanov, On Recursively Enumerable Structures, Forschungsberichte Mathematische Logik, Univ. Heidelberg, Math. Institut, Bericht Nr. 10, Juli 1994, p.1-20 (Section: Universal structures, p.8-10).

16. R. I. SoAre, Recursively Enumerable Sets and Degrees, Springer Verlag, BerlinHeidelberg-New York, 1987.

17. B. A. Trakntenbrot, The impossibility of an algorithm for the decision problem in finite domains, Dokl. Akad. Nauk SSSR, v.70, No.4, 1950, p. 569-572 (Russian).

18. R. L. VAught, Sentences true in all constructive models, J. Symbolic Logic, v. 25, No. 1, 1961, p. 39-58. 


\section{Contents}

1 The main results $\quad 2$

$\begin{array}{lll}2 & \text { Two numerations of tuples } & 7\end{array}$

3 Computations unfolded in time on a Turing machine 8

4 Design of a finitely axiomatizable theory 12

5 Programming technique $\quad 22$

6 Axiomatic and base properties of models 33

$\begin{array}{lll}7 & \text { Geometric properties of models } & 41\end{array}$

8 General form of a model $\quad 45$

9 Description of the regions of a model 49

10 Combinatorics of local and horizontal types 56

11 The Ehrenfeucht property $\quad 76$

12 References $\quad 84$ 